\title{
ASYMPTOTIC EXPANSIONS FOR THE POWER OF DISTRIBUTIONFREE TESTS IN THE TWO-SAMPLE PROBLEM ${ }^{1}$
}

\author{
By P. J. BICKEL ${ }^{2}$ AND W. R. VAN ZWET ${ }^{3}$ \\ University of California, Berkeley and \\ University of Leiden
}

\begin{abstract}
Asymptotic expansions are established for the power of distributionfree tests in the two-sample problem. These expansions are then used to obtain deficiencies in the sense of Hodges and Lehmann for distributionfree tests with respect to their parametric competitors and for the estimators of shift associated with these tests.
\end{abstract}

1. Introduction. Let $X_{1}, X_{2}, \cdots, X_{N}, N=m+n$, be independent random variables such that $X_{1}, \ldots, X_{m}$ are identically distributed with common distribution function $F$ and density $f$ and $X_{m+1}, \cdots, X_{N}$ are identically distributed with distribution function $G$ and density $g$. For $N=2,3, \cdots$ and $0<\varepsilon \leqq$ $m / N \leqq 1-\varepsilon<1$, consider the problem of testing the hypothesis $F=G$ against a sequence of alternatives that is contiguous to the hypothesis. The level $\alpha$ of the sequence of tests is fixed in $(0,1)$. Standard tests for this two-sample problem are linear rank tests and permutation tests and expressions for the limiting powers of such tests are well known. In this paper we shall establish asymptotic expansions to order $N^{-1}$ for the powers $\pi_{N}$ of such tests, i.e., expressions of the form $\pi_{N}=c_{0}+c_{1} N^{-\frac{1}{2}}+c_{2, N} N^{-1}+o\left(N^{-1}\right)$. Of course this involves finding similar expansions for the distribution function of the test statistic under the hypothesis as well as under contiguous alternatives. For simplicity we shall eventually limit our discussion to contiguous location alternatives. Extension of the results to general contiguous alternatives is straightforward but messy.

A number of authors have computed formal expansions for the distributions of various two-sample rank statistics without proof of their validity. Their purpose was to obtain better numerical approximations for the critical value of the test statistic and the power of the test than can be provided by the usual normal approximation. For an account of this work we refer to a review paper of Bickel

\footnotetext{
Received April 1976; revised March 1977.

${ }^{1}$ Report SW 38/76 (revised) Mathematisch Centrum, Amsterdam.

${ }^{2}$ Research supported by the National Science Foundation, Grant MPS-73-08698, by the Office of Naval Research, Contract N00014-75-C-0444, and by the Netherlands Organization for Pure Scientific Research.

${ }^{3}$ Research supported by the National Science Foundation, Grant GP-29123, and by the Office of Naval Research, Contracts N00014-69-A-0200-1036 and N00014-75-C-0444.

AMS 1970 subject classifications. Primary 62G10, 62G20; Secondary 60F05.

Key words and phrases. Distributionfree tests, linear rank tests, permutation test, HodgesLehmann estimators, power, contiguous alternatives, asymptotic expansions, Edgeworth expansions, deficiency, rejective sampling, sampling without replacement.
} 
(1974), which incidentally also contains a short preview of the present paper including a brief description of the expansion of the distribution function of the two-sample linear rank statistic under the hypothesis (cf. Corollary 2.1 in the present paper). This result was also proved independently by Robinson (1977). An earlier proof by Rogers (1971) for the special case of the two-sample Wilcoxon statistic under the hypothesis unfortunately appears to contain a nontrivial error.

We shall not discuss the numerical aspects of the expansions we obtain but we shall concentrate on a rather delicate type of asymptotic comparison of the power functions of various parametric and nonparametric tests. Consider two sequences of tests $\left\{T_{N}\right\}$ and $\left\{T_{N}{ }^{\prime}\right\}$ for the same hypothesis at the same fixed level $\alpha$. Let $\pi_{N}\left(\theta_{N}\right)$ and $\pi_{N}{ }^{\prime}\left(\theta_{N}\right)$ denote the powers of these tests against the same sequence of contiguous alternatives parametrized by a parameter $\theta$. If $T_{N}$ is more powerful than $T_{N}{ }^{\prime}$ we search for a number $k_{N}=N+d_{N}$ such that $\pi_{N}\left(\theta_{N}\right)=\pi_{k_{N}}^{\prime}\left(\theta_{N}\right)$. Here $k_{N}$ and $d_{N}$ are treated as continuous variables, the power $\pi_{N}{ }^{\prime}$ being defined for real $N$ by linear interpolation between consecutive integers. The quantity $d_{N}$ was named the deficiency of $\left\{T_{N}{ }^{\prime}\right\}$ with respect to $T_{N}$ by Hodges and Lehmann (1970), who introduced this concept and initiated its study. Of course, in many cases of interest $d_{N}$ is analytically intractable and one can only study its asymptotic behavior as $N$ tends to infinity.

Suppose that for $N \rightarrow \infty$, the ratio $N / k_{N}$ tends to a limit $e$, the asymptotic relative efficiency of $\left\{T_{N}{ }^{\prime}\right\}$ with respect to $\left\{T_{N}\right\}$. If $0<e<1$, we have $d_{N} \sim$ $\left(e^{-1}-1\right) N$ and further asymptotic information about $d_{N}$ is not particularly revealing. On the other hand, if $e=1$, the asymptotic behavior of $d_{N}$ (which may now be anything from $o(1)$ to $o(N)$ ) does provide important additional information. Of special interest is the case where $d_{N}$ tends to a finite limit.

Asymptotic expansions for the power of the type we discussed above are precisely what is needed for an asymptotic evaluation of $d_{v}$. With the aid of such expansions we arrive at the following results. Let $F$ be a distribution function with density $f$, let $b$ be a positive real number and define $\theta_{N}=b N^{-\frac{1}{2}}$. Consider the problem of testing the hypothesis $(F, F)$ against the sequence of simple alternatives $\left(F\left(\cdot+\Delta_{N} \theta_{N}\right), F\left(\cdot-\left(1-\Delta_{N}\right) \theta_{N}\right)\right)$ at level $\alpha$. Let $d_{N}$ denote the deficiency of the locally most powerful rank test with respect to the most powerful test for this problem. For the rank test the power is independent of $\Delta_{N}$ but for the most powerful test it is not and'we choose $\Delta_{N}$ in such a way that the power of the most powerful test is minimal. Under certain regularity conditions on $F$ we establish an expansion for $d_{y}$ with remainder $o(1)$. To indicate the qualitative behavior of $d_{N}$ it suffices to note that the expansion is of the form

$$
d_{N}=\frac{1}{\int \Psi_{1}^{2}(t) d t} \sum_{j=1}^{v} \sigma^{2}\left(\Psi_{1}\left(U_{j: N}\right)\right)+\tilde{d}_{N, 0}+o(1)
$$

where $\Psi_{1}=f^{\prime}\left(F^{-1}\right) / f\left(F^{-1}\right), \sigma^{2}$ indicates a variance, $U_{j: N}$ denotes the $j$ th order statistic of a sample of size $N$ from a uniform distribution on $(0,1)$ and 
$\tilde{d}_{N, 0}=O(1)$. Alternatively we may write

$$
\begin{aligned}
d_{N}= & \frac{1}{\int \Psi_{1}^{2}(t) d t} \int_{N^{-1}}^{1-N^{-1}}\left(\Psi_{1}^{\prime}(t)\right)^{2} t(1-t) d t+\tilde{d}_{N, 0}+o(1) \\
& +O\left(N^{-\frac{1}{2}} \int_{N^{-1}}^{1-N^{-1}}\left(\Psi_{1}{ }^{\prime}(t)\right)^{2}\{t(1-t)\}^{\frac{1}{2}} d t\right),
\end{aligned}
$$

where $\Psi_{1}^{\prime}$ is the derivative of $\Psi_{1}$. If we replace the exact scores $-E \Psi_{1}\left(U_{j: N}\right)$ in the locally most powerful rank test by the corresponding approximate scores $-\Psi_{1}(j /(N+1))$, then $(1.1)$ changes to

$$
d_{N}=\frac{1}{\int \Psi_{1}^{2}(t) d t} \sum_{j=1}^{N} E\left\{\Psi_{1}\left(U_{j: N}\right)-\Psi_{1}(j /(N+1))\right\}^{2}+d_{N, 0}+o(1)
$$

and (1.2) continues to hold. Thus the asymptotic behavior of $d_{N}$ is governed by that of the first term in these expansions and under the conditions imposed, all we can say is that it is $o\left(N^{\frac{1}{3}}\right)$ but not $o(1)$. Typically, however, it will be $O(1)$ or only slightly larger than that. By taking $F$ to be a normal distribution we find that the deficiency of both the normal scores test and van der Waerden's test with respect to the test based on the difference of the sample means for contiguous normal location alternatives is asymptotic to $\log \log N$. For logistic shift alternatives the deficiency of Wilcoxon's test with respect to the most powerful parametric test tends to a finite limit. Turning to distributionfree tests other than rank tests, we find that for contiguous normal location alternatives the deficiency of the permutation test based on the sample means with respect to Student's test tends to zero for $N \rightarrow \infty$.

If the locally most powerful rank test for shift has nondecreasing scores, then there exists a corresponding Hodges-Lehmann estimator of shift in the two-sample problem (cf. Hodges and Lehmann (1963)). There is a similar correspondence between the locally most powerful parametric test for shift and the maximum likelihood estimator of shift in the two-sample problem. We shall exploit this correspondence to obtain asymptotic expansions for the distribution functions of these estimators. We shall show that, when suitably defined, the deficiency of the Hodges-Lehmann estimator associated with the locally most powerful rank test with respect to the maximum likelihood estimator is asymptotically equivalent to the deficiency of the parent tests for $\alpha=\frac{1}{2}$.

This paper is thus the natural counterpart of Albers, Bickel and van Zwet (ABZ) (1976) where exactly the same programme is carried out for the onesample problem. Without exception the results are also qualitatively the same but contrary to what one might think at first sight, th is in itself is rather surprising. Of course there is a strong similarity between the one- and two-sample cases but there is also one major difference. In the nonparametric one-sample location problem the underlying distribution is always symmetric both under the hypothesis and under the alternative. Because of this symmetry, the power expansions for contiguous location alternatives do not contain a term of order 
$N^{-\frac{1}{2}}$ for any of the parametric or nonparametric tests considered. Since attention is restricted to sequences of tests $\left\{T_{N}\right\}$ and $\left\{T_{N}{ }^{\prime}\right\}$ with asymptotic relative efficiency 1 , the leading terms of the power expansions coincide and these expansions must therefore be of the form $\pi_{N}=c_{0}+c_{2, N} N^{-1}+o\left(N^{-1}\right)$ and $\pi_{N}{ }^{\prime}=c_{0}+c_{2, N}^{\prime} N^{-1}+o\left(N^{-1}\right)$. In the comparison of rank tests $T_{N}{ }^{\prime}$ with parametric tests $T_{N}$ it is found that the deficiency $d_{N}$ is of the order of $N\left(\pi_{N}-\pi_{N}{ }^{\prime}\right)=$ $\left(c_{2, N}-c_{2, N}^{\prime}\right)+o(1)=o\left(N^{\frac{1}{3}}\right)$. In the two-sample problem, however, the underlying distributions are not required to be symmetric and as a result the power expansions do in general contain a term of order $N^{-\frac{1}{2}}$. It is not clear a priori that this term should be the same in each expansion and because $d_{N}$ is again of the order of $N\left(\pi_{N}-\pi_{N}{ }^{\prime}\right)$, one should expect $d_{N}$ to be of the order $N^{\frac{1}{2}}$. It turns out, however, that for the most powerful test, the locally most powerful test, the locally most powerful rank test and its approximate scores analogue, the term of order $N^{-\frac{1}{2}}$ in the power expansion for contiguous location alternatives is in fact the same for each of these four tests. Borrowing a phrase from Pfanzagl (1977) who noted the same phenomenon for the (asymmetric) parametric onesample problem, first order efficiency apparently implies second order efficiency in these cases. It follows that again $d_{N}$ is of the order of $\left(c_{2, N}-c_{2, N}^{\prime}\right)$ and since $c_{2, N}$ and $c_{2, N}^{\prime}$ exhibit precisely the same asymptotic behavior as in the one-sample case, our deficiency results are qualitatively the same as in ABZ (1976). The reader should note that Pfazagl's concept of second order efficiency which in general implies $d_{v}=o\left(N^{\frac{1}{2}}\right)$, is different from Rao's concept of second order efficiency as discussed in Efron (1975), which is more in the nature of $d_{N}=o(1)$. This difference in terminology is not as illogical as it may seem because Rao's concept is related to the asymptotic performance of an estimator $M_{N}$ as measured by the asymptotic variance of $N^{\frac{1}{2}} M_{N}$ and expansions for this quantity are typically in powers of $N^{-1}$ rather than $N^{-\frac{1}{2}}$.

Throughout this paper we shall draw heavily on the techniques developed for the one-sample case in ABZ (1976) but several new difficulties appear that make the two-sample case essentially more complicated. The main source of trouble is the occurrence of terms of order $N^{-\frac{1}{2}}$ in our expansions. Not only do they make the actual computation of the expansions much more laborious, but their presence also poses a number of technical problems that are hard to handle under the conditions imposed, which are comparable to those in $\mathrm{ABZ}$ (1976). Another complicating factor is that the distribution theory for the twosample rank statistic is more involved than for its one-sample counterpart. In the one-sample case a conditioning argument reduces the rank statistic to a weighted sum of independent Bernoulli random variables. A similar argument in the two-sample case leads to the much less manageable random variable indicated below.

In Section 2 we point out that for arbitrary $F$ and $G$, the conditional distribution of the two-sample linear rank statistic given the order statistics of the combined sample is the same as the distribution of the sample sum in a rejective 
sampling scheme. We establish an expansion for the distribution function of such a sample sum which may be of interest in its own right. As a corollary we obtain an expansion for the distribution function of the rank statistic under the hypothesis. In Section 3 we return to general $F$ and $G$ and obtain an unconditional expansion for the distribution function of the rank statistic. We specialize to contiguous location alternatives in Section 4 and derive an expansion for the power of the rank test. In Section 5 we deal with the important case where the scores are exact or approximate scores generated by a smooth function. The permutation test based on the sample means is discussed in Section 6. The results on deficiencies of distributionfree tests are contained in Section 7. Section 8 is devoted to estimators. Some technical results are dealt with in the appendix.

2. An expansion for the conditional distribution of two-sample rank statistics and its application to rejective sampling. Let $X_{1}, X_{2}, \cdots, X_{N}, N=m+n$, be independent random variables (rv's) such that $X_{1}, \ldots, X_{m}$ are identically distributed (i.d.) with common distribution function (df) $F$ and density $f$ and $X_{m+1}, \cdots, X_{N}$ are i.d. with common df $G$ and density $g$. Let $Z_{1}<Z_{2}<\cdots<Z_{N}$ denote the order statistics of $X_{1}, \cdots, X_{N}$, define the antiranks $D_{1}, D_{2}, \cdots, D_{N}$ by $X_{D_{j}}=Z_{j}$ and let

$$
\begin{aligned}
V_{j} & =1 & & \text { if } \quad m+1 \leqq D_{j} \leqq N \\
& =0 & & \text { otherwise. }
\end{aligned}
$$

For a specified vector of scores $a=\left(a_{1}, a_{2}, \ldots, a_{N}\right)$ define a two-sample rank statistic by

$$
T=\sum_{j=1}^{N} a_{j} V_{j}
$$

Our aim is to obtain an asymptotic expansion as $N \rightarrow \infty$ for the distribution of $T$ for suitable sequences of pairs of df's $\left(F_{N}, G_{N}\right)$, arrays of scores $\left\{a_{j, N}\right\}, 1 \leqq$ $j \leqq N$, and sample sizes $\left(m_{N}, n_{N}\right)$. As in Albers, Bickel and van Zwet (ABZ) (1976) we shall suppress dependence on $N$ whenever possible and formally present our results in terms of error bounds for fixed, but arbitrary, values of $N$.

Under the null-hypothesis that $F=G$,

$$
P\left(V_{1}=v_{1}, \cdots, V_{N}=v_{N}\right)=\frac{1}{\left(\begin{array}{l}
N \\
n
\end{array}\right)}
$$

for any vector $\left(v_{1}, \ldots, v_{N}\right)$ with $m$ coordinates equal to 0 and $n$ coordinates equal to 1 . In general, conditional on $Z=\left(Z_{1}, \cdots, Z_{N}\right)$,

$$
P\left(V_{1}=v_{1}, \cdots, V_{N}=v_{N} \mid Z\right)=c^{-1}(P) \prod_{j=1}^{N} P_{j}^{v_{j}}\left(1-P_{j}\right)^{1-v_{j}},
$$

where

$$
P_{j}=\frac{\lambda g\left(Z_{j}\right)}{(1-\lambda) f\left(Z_{j}\right)+\lambda g\left(Z_{j}\right)},
$$




$$
\begin{gathered}
\lambda=\frac{n}{N}, \\
c(P)=\sum \prod_{j=1}^{N} P_{j}{ }^{w_{j}}\left(1-P_{j}\right)^{1-w_{j}},
\end{gathered}
$$

and the summation is over all vectors $\left(w_{1}, \ldots, w_{N}\right)$ consisting of $m$ zeros and $n$ ones.

Let $W_{1}, W_{2}, \cdots, W_{N}$ be independent rv's with $P\left(W_{j}=1\right)=1-P\left(W_{j}=0\right)=p_{j}$, $1 \leqq j \leqq N$. Suppose that

$$
\begin{array}{llll}
p_{j}=0 & \text { for at most } & m \text { indices } & j \\
p_{j}=1 & \text { for at most } n \text { indices } & j
\end{array}
$$

and consider the conditional distribution of $\sum a_{j} W_{j}$ given that $\sum W_{j}=n$. Note that if we replace $p=\left(p_{1}, \cdots, p_{N}\right)$ by $P=\left(P_{1}, \ldots, P_{N}\right)$, then this is the distribution of $T$ given $Z$. For general $p$ this distribution is of interest in its own right since $\sum a_{j} W_{j}$ given $\sum W_{j}=n$ is the sample sum we obtain when we use a rejective sampling scheme with parameters $p_{1}, \ldots, p_{N}$ in selecting a sample of size $n$ from the sampling frame $\left\{a_{1}, a_{2}, \cdots, a_{N}\right\}$ (see Hájek (1964) for details).

Define

$$
\begin{gathered}
\rho(t, p)=E\left(\exp \left\{i t N^{-\frac{1}{2}} \sum_{j=1}^{N} a_{j}\left(W_{j}-p_{j}\right)\right\} \mid \sum_{j=1}^{N} W_{j}=n\right), \\
R(x, p)=P\left(N^{-\frac{1}{2}} \sum_{j=1}^{N} a_{j}\left(W_{j}-p_{j}\right) \leqq x \mid \sum_{j=1}^{N} W_{j}=n\right) .
\end{gathered}
$$

Our program for obtaining an Edgeworth expansion for the df of $T$ parallels in part that of ABZ (1976). We obtain a formula for $\rho$. From this formula we obtain an expansion for $\rho$ which we can rigorously translate into an Edgeworth expansion for $R$. Because of the connection with rejective sampling we isolate this result as the only theorem in this section. In the next section we proceed with our main program and obtain an expansion for the $\mathrm{df}$ of $T$ by replacing $P$ by $P$ and taking the expectation of the resulting expression. We begin with

Lemma 2.1. Define

$$
\begin{gathered}
\psi(s, t, p)=\exp \left\{i s N^{-\frac{1}{2}} \sum_{j=1}^{N}\left(p_{j}-\lambda\right)\right\} \prod_{j=1}^{N}\left[p_{j} \exp \left\{i N^{-\frac{1}{2}}\left(1-p_{j}\right)\left(s+a_{j} t\right)\right\}\right. \\
\left.+\left(1-p_{j}\right) \exp \left\{-i N^{-\frac{1}{2}} p_{j}\left(s+a_{j} t\right)\right\}\right], \\
\nu(t, p)=\int_{-\pi N^{\frac{1}{2}}}^{\pi N^{\frac{1}{2}}} \psi(s, t, p) d s, \\
c(p)=\sum \prod_{j=1}^{N} p_{j}{ }^{w} j\left(1-p_{j}\right)^{1-w_{j}},
\end{gathered}
$$

where the last summation is over all vectors $\left(w_{1}, \cdots, w_{N}\right)$ consisting of $m$ zeros and $n$ ones. Then, if (2.7) is satisfied,

$$
\rho(t, p)=\frac{1}{2 \pi c(p) N^{\frac{1}{2}}} \int_{-\pi N^{\frac{1}{2}}}^{\pi N^{\frac{1}{2}}} \psi(s, t, p) d s=\frac{\nu(t, p)}{\nu(0, p)} .
$$

Proof. Begin with the identity

$$
\begin{gathered}
E\left(\exp \left\{i N^{-\frac{1}{2}}\left[s \sum\left(W_{j}-p_{j}\right)+t \sum a_{j}\left(W_{j}-p_{j}\right)\right]\right\}\right) \\
=\sum_{k=0}^{N} E\left(\exp \left\{i t N^{-\frac{1}{2}} \sum a_{j}\left(W_{j}-p_{j}\right)\right\} \mid \sum W_{j}=k\right) \\
\times P\left(\sum W_{j}=k\right) \exp \left\{i s N^{-\frac{1}{2}}\left(k-\sum p_{j}\right)\right\}
\end{gathered}
$$


Because the system $\left\{\left(2 \pi N^{\frac{1}{2}}\right)^{-1} \exp \left(i k s N^{-\frac{1}{2}}\right): k=0, \pm 1, \cdots\right\}$ is orthonormal on $\left[-\pi N^{\frac{1}{2}}, \pi N^{\frac{1}{2}}\right]$ this implies

$$
\begin{aligned}
\rho(t, p)=( & \left.2 \pi N^{\frac{1}{2}} P\left(\sum W_{j}=n\right)\right)^{-1} \int_{-\pi N^{\frac{1}{2}}}^{\pi N^{\frac{1}{2}}} \exp \left\{i s N^{-\frac{1}{2}} \sum\left(p_{j}-\lambda\right)\right\} \\
& \times E\left(\exp \left\{i N^{-\frac{1}{2}} \sum\left(s+a_{j} t\right)\left(W_{j}-p_{j}\right)\right\}\right) d s .
\end{aligned}
$$

Elementary considerations now yield (2.13).

Note that if $p_{j}=\lambda$ for all $j$ (which corresponds to the null-hypothesis in the two-sample problem) our formula agrees with that of Erdös and Rényi for random sampling without replacement (cf. Rényi (1970), page 462). In fact their result motivated our approach.

In our asymptotic study of $\psi, \nu$ and $\rho$ we shall repeatedly come across the following functions of $p$.

$$
\begin{gathered}
\omega(p)=N^{-\frac{1}{2}} \sum_{j=1}^{N}\left(p_{j}-\lambda\right), \\
\sigma^{2}(p)=N^{-1} \sum_{j=1}^{N} p_{j}\left(1-p_{j}\right), \\
\vec{a}(p)=\sum_{j=1}^{N} p_{j}\left(1-p_{j}\right) a_{j} / \sum_{j=1}^{N} p_{j}\left(1-p_{j}\right), \\
\tau^{2}(p)=N^{-1} \sum_{j=1}^{N} p_{j}\left(1-p_{j}\right)\left(a_{j}-\bar{a}(p)\right)^{2} \\
=N^{-1} \sum_{j=1}^{N} p_{j}\left(1-p_{j}\right) a_{j}{ }^{2}-\sigma^{2}(p) \bar{a}^{2}(p), \\
\kappa_{3, i}(p)=N^{-1} \sum_{j=1}^{N} p_{j}\left(1-p_{j}\right)\left(1-2 p_{j}\right)\left(a_{j}-\bar{a}(p)\right)^{i}, \quad i=0,1,2,3, \\
\kappa_{4, i}(p)=N^{-1} \sum_{j=1}^{N} p_{j}\left(1-p_{j}\right)\left(1-6 p_{j}+6 p_{j}{ }^{2}\right)\left(a_{j}-\bar{a}(p)\right)^{i}, \\
\quad i=0,1, \ldots, 4 .
\end{gathered}
$$

In this notation we shall suppress the dependence on $p$ when this is convenient. Let $l$ denote Lebesgue measure on $R^{1}$ and define

$$
\gamma(\varepsilon, \zeta, p)=l\left\{x: \exists j\left|x-a_{j}\right|<\zeta, \varepsilon \leqq p_{j} \leqq 1-\varepsilon\right\} .
$$

Lemma 2.2. Suppose that positive numbers $c, C, \delta$ and $\varepsilon$ exist such that

$$
\begin{gathered}
\tau^{2}(p) \geqq c, \quad \frac{1}{N} \sum_{j=1}^{N} a_{j}{ }^{4} \leqq C, \\
\gamma(\varepsilon, \zeta, p) \geqq \delta N \zeta \quad \text { for some } \zeta \geqq N^{-\frac{3}{2}} \log N .
\end{gathered}
$$

Then there exist positive numbers $b, B$ and $\beta$ depending only on $c, C, \delta$ and $\varepsilon$ such that

$$
|\psi(s, t, p)| \leqq B N^{-\beta \log N}
$$

for all pairs $(s, t)$ such that $|s| \leqq \pi N^{\frac{1}{2}},|t| \leqq b N^{\frac{3}{2}}$ and either $|s| \geqq \log (N+1)$ or $|t| \geqq \log (N+1)$.

PROOF.

$$
\begin{aligned}
|\psi(s, t, p)|= & \prod_{j=1}^{N}\left[1-2 p_{j}\left(1-p_{j}\right)\left\{1-\cos \left(N^{-\frac{1}{2}}\left(s+a_{j} t\right)\right)\right\}\right]^{\frac{1}{2}} \\
\leqq & \exp \left\{-\sum_{j=1}^{N} p_{j}\left(1-p_{j}\right)\left[\frac{1}{2} N^{-1}\left(s+a_{j} t\right)^{2}-\frac{1}{2} \frac{1}{4} N^{-2}\left(s+a_{j} t\right)^{4}\right]\right\} \\
\leqq & \exp \left\{-\frac{1}{2}\left[\tau^{2} t^{2}+\sigma^{2}(s+\bar{a} t)^{2}\right]\right. \\
& \left.\quad+\frac{1}{12} N^{-1}\left[N^{-1} \sum_{j=1}^{N}\left(a_{j}-\bar{a}\right)^{4} t^{4}+(s+\bar{a} t)^{4}\right]\right\} .
\end{aligned}
$$


Now (2.21) ensures that

$$
\begin{gathered}
\sigma^{2}(p) \geqq N \tau^{4}(p) / \sum_{j=1}^{N} a_{j}^{4} \geqq C^{-1} c^{2}, \\
|\bar{a}(p)| \leqq\left[N^{-1} \sum_{j=1}^{N} a_{j}^{4}\right]^{\frac{1}{4}} / \sigma^{2}(p) \leqq c^{-2} C^{\frac{4}{4}},
\end{gathered}
$$

and by (2.21), (2.24), (2.25) and (2.26) we conclude that there exist positive $b_{1}$, $B$ and $\beta$ depending only on $c$ and $C$ such that for $|s| \leqq b_{1} N^{\frac{1}{2}}$ and $|t| \leqq b_{1} N^{\frac{1}{2}}$

$$
|\psi(s, t, p)| \leqq B \exp \left\{-\beta\left(s^{2}+t^{2}\right)\right\} .
$$

Next note that (2.25) and (2.21) imply that the number of indices $j$ for which $p_{j}\left(1-p_{j}\right) \geqq \frac{1}{2} c^{2} / C$ is at least $2 N c^{2} / C$ and the number of $j$ for which $\left|a_{j}\right| \leqq(C / c)^{\frac{1}{2}}$ is at least $N-N c^{2} / C$. Hence the number of indices $j$ for which $\left|a_{j}\right| \leqq(C / c)^{\frac{1}{2}}$ and $p_{j}\left(1-p_{j}\right) \geqq \frac{1}{2} c^{2} / C$ is at least $N c^{2} / C$. Put $b_{2}=\frac{1}{2} b_{1}(c / C)^{\frac{1}{2}}$ and we see that if $b_{1} N^{\frac{1}{2}} \leqq|s| \leqq \pi N^{\frac{1}{2}}$ and $|t| \leqq b_{2} N^{\frac{1}{2}}$, then for at least $N c^{2} / C$ indices $j$

$$
\left[1-2 p_{j}\left(1-p_{j}\right)\left\{1-\cos \left(N^{-\frac{1}{2}}\left(s+a_{j} t\right)\right)\right\}\right] \leqq 1-c^{2} C^{-1}\left\{1-\cos \left(\frac{b_{1}}{2}\right)\right\} .
$$

Combining this with (2.27) we see that it only remains to be shown that positive numbers $b, B$ and $\beta$ exist depending only on $c, C, \delta$ and $\varepsilon$ and such that (2.23) holds for $|s| \leqq \pi N^{\frac{1}{2}}$ and $\left(b_{1} \wedge b_{2}\right) N^{\frac{1}{2}} \leqq|t| \leqq b N^{\frac{3}{2}}$. For this we can appeal to the corresponding part of the proof of Lemma 2.2 in ABZ (1976) with only minor modifications.

Define functions $\mu_{k}(p), 1 \leqq k \leqq 6$, and $A_{k}(p), 0 \leqq k \leqq 6$, by

$$
\begin{gathered}
\mu_{1}=\frac{\omega}{\sigma^{2}}, \quad \mu_{2}=\frac{1}{\sigma^{2}}-\frac{\omega^{2}}{\sigma^{4}}, \quad \mu_{3}=\frac{3 \omega}{\sigma^{4}}-\frac{\omega^{3}}{\sigma^{6}}, \\
\mu_{4}=\frac{3}{\sigma^{4}}-\frac{6 \omega^{2}}{\sigma^{6}}+\frac{\omega^{4}}{\sigma^{8}}, \quad \mu_{5}=\frac{15 \omega}{\sigma^{6}}-\frac{10 \omega^{3}}{\sigma^{8}}+\frac{\omega^{5}}{\sigma^{10}}, \\
\mu_{6}=\frac{15}{\sigma^{6}}-\frac{45 \omega^{2}}{\sigma^{8}}+\frac{15 \omega^{4}}{\sigma^{10}}-\frac{\omega^{6}}{\sigma^{12}}, \\
A_{0}=1+\frac{N^{-\frac{1}{2}}}{6} \kappa_{3,0} \mu_{3}+\frac{N^{-1}}{72}\left(3 \kappa_{4,0} \mu_{4}-\kappa_{3,0}^{2} \mu_{6}\right), \\
A_{1}=-\frac{N^{-\frac{1}{2}}}{2} \kappa_{3,1} \mu_{2}+\frac{N^{-1}}{12}\left(2 \kappa_{4,1} \mu_{3}-\kappa_{3,0} \kappa_{3,1} \mu_{5}\right), \\
A_{2}=-\frac{N^{-\frac{1}{2}}}{2} \kappa_{3,2} \mu_{1}+\frac{N^{-1}}{24}\left\{-6 \kappa_{4,2} \mu_{2}+\left(2 \kappa_{3,0} \kappa_{3,2}+3 \kappa_{3,1}^{2}\right) \mu_{4}\right\}, \\
A_{3}=\frac{N^{-\frac{1}{2}}}{6} \kappa_{3,3}+\frac{N^{-1}}{36}\left\{-6 \kappa_{4,3} \mu_{1}+\left(\kappa_{3,0} \kappa_{3,3}+9 \kappa_{3,1} \kappa_{3,2}\right) \mu_{3}\right\}, \\
A_{4}=\frac{N^{-1}}{24}\left\{\kappa_{4,4}-\left(2 \kappa_{3,1} \kappa_{3,3}+3 \kappa_{3,2}^{2}\right) \mu_{2}\right\}, \\
A_{5}=-\frac{N^{-1}}{12} \kappa_{3,2} \kappa_{3,3} \mu_{1}, \\
A_{6}=\frac{N^{-1}}{72} \kappa_{3,3}^{2},
\end{gathered}
$$


where we have suppressed the dependence on $p$. We shall show that

$$
\tilde{\nu}(t, p)=\frac{(2 \pi)^{\frac{1}{2}}}{\sigma(p)} \exp \left\{-\frac{\omega^{2}(p)}{2 \sigma^{2}(p)}-\frac{\tau^{2}(p) t^{\boldsymbol{x}}}{2}-i \omega(p) \bar{a}(p) t\right\} \sum_{k=0}^{6} A_{k}(p)(i t)^{k}
$$

is an asymptotic expansion for $\nu(t, p)$.

Lemma 2.3. Suppose that positive numbers $c, C, \delta$ and $\varepsilon$ exist such that (2.21) and (2.22) are satisfied. Then there exist positive numbers $b, B$ and $\beta$ depending only on $c, C, \delta$ and $\varepsilon$ such that for $|t| \leqq b N^{\frac{3}{2}}$,

$$
|\mathcal{\nu}(t, p)-\tilde{\nu}(t, p)| \leqq B\left[\left(N^{-\frac{3}{2}}+N^{-\frac{5}{4}}|t|^{5}\right) \exp \left\{-\frac{c t^{2}}{8}\right\}+N^{-\beta \log x}\right] .
$$

Proof. In this proof $b, b_{i}, B_{i}, \beta_{i}$ and $N_{0}$ denote appropriately chosen positive numbers depending only on $c, C, \delta$ and $\varepsilon$.

Arguing as in the proof of Theorem 2.1 in ABZ (1976) we find by Taylor expansion of $\log \psi$ that if $\left|s+a_{j} t\right| \leqq \frac{1}{2} \pi N^{\frac{1}{2}}$ for all $j$, then

$$
\begin{aligned}
\phi(s, t, p) & \\
= & \exp \left\{i \omega s-\frac{\tau^{2} t^{2}}{2}-\frac{\sigma^{2}(s+\bar{a} t)^{2}}{2}\right. \\
& -\frac{i N^{-\frac{3}{2}}}{6} \sum p_{j}\left(1-p_{j}\right)\left(1-2 p_{j}\right)\left(s+a_{j} t\right)^{3} \\
& \left.+\frac{N^{-2}}{24} \sum p_{j}\left(1-p_{j}\right)\left(1-6 p_{j}+6 p_{j}{ }^{2}\right)\left(s+a_{j} t\right)^{4}+M_{1}(s, t, p)\right\},
\end{aligned}
$$

where

$$
\begin{aligned}
\left|M_{1}(s, t, p)\right| & \leqq C_{1} N^{-\frac{5}{2}} \sum\left|s+a_{j} t\right|^{5} \\
& \leqq 16 C_{1}\left(N^{-\frac{5}{2}}|t|^{5} \sum\left|a_{j}-\bar{a}\right|^{5}+N^{-\frac{3}{2}}|s+\bar{a} t|^{5}\right)
\end{aligned}
$$

for some absolute constant $C_{1}$. Now (2.21) and (2.26) imply that $N^{-1} \sum\left|a_{j}-\bar{a}\right|^{3}$, $N^{-1} \sum\left|a_{j}-\bar{a}\right|^{4}, N^{-\frac{1}{4}} \max \left|a_{j}\right|$ and $N^{-\frac{5}{5}} \sum\left|a_{j}-\bar{a}\right|^{5}$ are bounded. Using (2.21) and (2.25) we find that for all $|s| \leqq b_{1} N^{\frac{1}{2}}$ and $|t| \leqq b_{1} N^{\frac{1}{4}}$

$$
\frac{N^{-\frac{3}{2}}}{6} \sum\left|s+a_{j} t\right|^{3}+\frac{N^{-2}}{24} \sum\left(s+a_{j} t\right)^{4}+\left|M_{1}(s, t, p)\right| \leqq \frac{\tau^{2} t^{2}+\sigma^{2}(s+\bar{a} t)^{2}}{4} \text {. }
$$

Hence further expansion of part of the exponential in (2.32) shows that

$$
\phi(s, t, p)=\tilde{\psi}(s, t, p)+M_{2}(s, t, p)
$$

for $|s| \leqq b_{1} N^{\frac{1}{2}}$ and $|t| \leqq b_{1} N^{\frac{1}{b}}$, where

$$
\begin{aligned}
\tilde{\psi}(s, t, p)=\exp & \left\{i \omega s-\frac{\tau^{2} t^{2}}{2}-\frac{\sigma^{2}(s+\bar{a} t)^{2}}{2}\right\} \\
& \times\left[1-\frac{i N^{-\frac{3}{2}}}{6} \sum p_{j}\left(1-p_{j}\right)\left(1-2 p_{j}\right)\left(s+a_{j} t\right)^{3}\right. \\
& +\frac{N^{-2}}{24} \sum p_{j}\left(1-p_{j}\right)\left(1-6 p_{j}+6 p_{j}{ }^{2}\right)\left(s+a_{j} t\right)^{4} \\
& \left.-\frac{N^{-3}}{72}\left(\sum p_{j}\left(1-p_{j}\right)\left(1-2 p_{j}\right)\left(s+a_{j} t\right)^{3}\right)^{2}\right],
\end{aligned}
$$




$$
\left|M_{2}(s, t, p)\right| \leqq\left(N^{-\frac{3}{2}}+N^{-\frac{5}{4}}|t|^{5}\right) M_{3}(t, s+\bar{a} t) \exp \left\{-\frac{\tau^{2} t^{2}+\sigma^{2}(s+\bar{a} t)^{2}}{4}\right\}
$$

and $M_{3}$ is a polynomial in $t$ and $(s+\bar{a} t)$ of fixed degree with coefficients depending only on $c$ and $C$. Therefore, for $|t| \leqq b_{1} N^{\frac{1}{4}}$,

$$
\int_{-b_{1} N^{\frac{1}{2}}}^{b_{1} N^{\frac{1}{2}}}|\psi(s, t, p)-\tilde{\psi}(s, t, p)| d s \leqq B_{1}\left(N^{-\frac{3}{2}}+N^{-\frac{5}{4}}|t|^{5}\right) \exp \left\{-\frac{c t^{2}}{8}\right\} .
$$

Next we show that for $|t| \leqq b_{1} N^{\frac{1}{4}}$,

$$
\begin{gathered}
\int_{b_{1} N^{\frac{1}{2}} \leqq|s| \leqq \pi N^{\frac{1}{2}}}|\psi(s, t, p)| d s \leqq B_{2} N^{-\beta_{2} \log N}, \\
\int_{|s| \geqq 1^{N^{\frac{1}{2}}}}|\tilde{\psi}(s, t, p)| d s \leqq B_{3} N^{-\beta_{3} \log N} .
\end{gathered}
$$

For $N \geqq N_{0},(2.37)$ is a consequence of Lemma 2.2 and since $|\psi| \leqq 1$ we can choose $B_{2}$ so that $(2.37)$ holds for all $N$. Because for all $s$ and $t$

$$
|\tilde{\psi}(s, t, p)| \leqq \exp \left\{\frac{\tau^{2} t^{2}+\sigma^{2}(s+\bar{a} t)^{2}}{2}\right\} M_{4}(t, s+\bar{a} t)
$$

where $M_{4}$ is a polynomial depending only on $c$ and $C,(2.38)$ follows. Combining (2.11), (2.36), (2.37) and (2.38) we see that for $|t| \leqq b_{1} N^{\frac{1}{4}}$

$$
\begin{aligned}
\mid \mathcal{L}(t, p)- & \int_{-\infty}^{\infty} \tilde{\phi}(s, t, p) d s \mid \\
& \leqq B_{4}\left[\left(N^{-\frac{3}{2}}+N^{-\frac{5}{4}}|t|^{5}\right) \exp \left\{-\frac{c t^{2}}{8}\right\}+N^{-\beta_{4} \log N}\right] .
\end{aligned}
$$

A direct application of Lemma 2.2, the fact that $|\psi| \leqq 1$ and (2.39) show that we can choose $B_{4}$ and $\beta_{4}$ so that $(2.40)$ continues to hold for $b_{1} N^{\frac{1}{4}} \leqq|t| \leqq b N^{\frac{3}{2}}$ with $b$ as in Lemma 2.2.

It remains to be shown that for all $s$ and $t$

$$
\tilde{\mathcal{L}}(t, p)=\int_{-\infty}^{\infty} \tilde{\psi}(s, t, p) d s .
$$

This follows by straightforward but tedious computation using the fact that

$$
\begin{array}{rlrl}
(2 \pi)^{-\frac{1}{2}} \int_{-\infty}^{\infty}\left(\frac{z}{\sigma(p)}+\frac{i \omega(p)}{\sigma^{2}(p)}\right)^{k} e^{-\frac{1}{2} z^{2}} d z & =\mu_{k}(p) & \text { for even } k \\
& =i \mu_{k}(p) \quad \text { for odd } k .
\end{array}
$$

We now turn to our asymptotic expansion for rejective sampling. For $1 \leqq k \leqq 6$, define functions $Q_{k}(p)$ by

$$
\begin{aligned}
& Q_{1}=-\frac{N^{-\frac{1}{2}}}{2} \kappa_{3,1} \mu_{2}+\frac{N^{-1}}{6}\left[\kappa_{4,1} \mu_{3}-3 \kappa_{3,0} \kappa_{3,1}\left(2 \frac{\omega}{\sigma^{6}}-\frac{\omega^{3}}{\sigma^{8}}\right)\right], \\
& Q_{2}=-\frac{N^{-\frac{1}{2}}}{2} \kappa_{3,2} \mu_{1}+\frac{N^{-1}}{8}\left[-2 \kappa_{4,2} \mu_{2}+2 \kappa_{3,0} \kappa_{3,2}\left(\frac{1}{\sigma^{4}}-\frac{\omega^{2}}{\sigma^{6}}\right)+\kappa_{3,1}^{2} \mu_{4}\right], \\
& Q_{3}=\frac{N^{-\frac{1}{2}}}{6} \kappa_{3,3}+\frac{N^{-1}}{12}\left[-2 \kappa_{4,3} \mu_{1}+3 \kappa_{3,1} \kappa_{3,2} \mu_{3}\right], \\
& Q_{k}=A_{k}, \quad k=4,5,6 .
\end{aligned}
$$


Let $\Phi$ and $\phi$ denote the standard normal df and its density and let $H_{k}$ denote the Hermite polynomial of degree $k$, thus

$$
\begin{aligned}
& H_{0}(x)=1, \quad H_{1}(x)=x, \quad H_{2}(x)=x^{2}-1, \quad H_{3}(x)=x^{3}-3 x, \\
& H_{4}(x)=x^{4}-6 x^{2}+3, \quad H_{5}(x)=x^{5}-10 x^{3}+15 x .
\end{aligned}
$$

We shall show that expansions for (2.8) and (2.9) are given by

$$
\begin{aligned}
\tilde{\rho}(t, p)=\exp & \left\{-\frac{\tau^{2}(p) t^{2}}{2}-i \omega(p) \bar{a}(p) t\right\}\left[1+\sum_{k=1}^{6} Q_{k}(p)(i t)^{k}\right], \\
\tilde{R}(x, p)=\Phi & \left(\frac{x+\omega(p) \bar{a}(p)}{\tau(p)}\right)-\phi\left(\frac{x+\omega(p) \bar{a}(p)}{\tau(p)}\right) \sum_{k=1}^{6} \frac{Q_{k}(p)}{(\tau(p))^{k}} \\
& \times H_{k-1}\left(\frac{x+\omega(p) \bar{a}(p)}{\tau(p)}\right) .
\end{aligned}
$$

Note that $\tilde{\rho}$ is the Fourier-Stieltjes transform of $\tilde{R}$, i.e., $\tilde{\rho}(t, p)=\int e^{i t x} d \tilde{R}(x, p)$.

THEOREM 2.1. Suppose that positive numbers $c, C, D, \delta$ and $\varepsilon$ exist such that (2.21) and (2.22) are satisfied and

$$
|\omega(p)| \leqq D
$$

Then there exist positive numbers $N_{0}$ and $B$ depending only on $c, C, D, \delta$ and $\varepsilon$ such that for $N \geqq N_{0}, R(x, p)$ is well defined and

$$
\sup _{x}|R(x, p)-\tilde{R}(x, p)| \leqq B N^{-5} .
$$

Proof. In this proof $b, B_{i}, \beta, \eta$ and $N_{0}$ denote appropriately chosen positive numbers depending only on $c, C, D, \delta$ and $\varepsilon$.

By (2.21), (2.25), (2.26), (2.46) and Lemma 2.3 we have for $N \geqq N_{0}$,

$$
|\tilde{\nu}(0, p)| \geqq \eta, \quad|\nu(0, p)-\tilde{\nu}(0, p)| \leqq \frac{\eta}{2},
$$

so that $|\nu(0, p)| \geqq \eta / 2>0$. In the first place it follows that for $N \geqq N_{0}, c(p)>0$ and hence $(2.7)$ is satisfied and $R(x, p)$ is properly defined. We assume that $N \geqq N_{0}$ and we shall show that, with $b$ as in Lemma 2.3,

$$
\int_{-b N^{\frac{3}{2}}}^{b N^{\frac{3}{2}}}\left|\frac{\rho(t, p)-\tilde{\rho}(t, p)}{t}\right| d t \leqq B_{1} N^{-\frac{5}{4}} .
$$

By Esseen's smoothing lemma (Esseen (1945)) this suffices to prove the theorem because $\tilde{R}(-\infty, p)=0, \tilde{R}(\infty, p)=1$ and the derivative of $\tilde{R}$ with respect to $x$ is bounded.

By (2.21), (2.25), (2.26) and (2.46), $\tilde{\rho}$ has a bounded derivative with respect to $t$. Also

$$
\left|\frac{d \rho(t, p)}{d t}\right| \leqq N^{-\frac{1}{2}} E\left(\left|\sum a_{j}\left(W_{j}-p_{j}\right)\right| \mid \sum W_{j}=n\right) \leqq N^{-\frac{1}{2}} \sum\left|a_{j}\right| \leqq C^{\frac{1}{4}} N^{\frac{1}{2}} .
$$

Since $\rho(0, p)=\tilde{\rho}(0, t)=1$, it follows that

$$
\int_{-N^{-2}}^{N^{-2}}\left|\frac{\rho(t, p)-\tilde{\rho}(t, p)}{t}\right| d t \leqq B_{2} N^{-\frac{3}{2}}
$$


Next we note that (2.21), (2.25) and (2.26) ensure that for all $t$

$$
|\tilde{\nu}(t, p)| \leqq B_{3} \exp \left\{-\frac{c t^{2}}{4}\right\}
$$

Together with (2.13), (2.48) and Lemma 2.3 this implies that for $|t| \leqq b N^{\frac{3}{2}}$

$$
\begin{aligned}
\left|\rho(t, p)-\frac{\tilde{\nu}(t, p)}{\tilde{\nu}(0, p)}\right| & \leqq \frac{2}{\eta}|\nu(t, p)-\tilde{\nu}(t, p)|+\frac{2}{\eta^{2}}|\tilde{\nu}(t, p)||\nu(0, p)-\tilde{\nu}(0, p)| \\
& \leqq B_{4}\left[\left(N^{-\frac{3}{2}}+N^{-\frac{5}{4}}|t|^{5}\right) \exp \left\{-\frac{c t^{2}}{8}\right\}+N^{-\beta \log N}\right] .
\end{aligned}
$$

Again with the aid of (2.21), (2.25) (2.26) and (2.46) one can easily check that, for $1 \leqq k \leqq 6, Q_{k}$ is obtained from $A_{k} / A_{0}$ by expanding the denominator and discarding all terms of order $N^{-\frac{3}{2}}$, i.e., that $\left|Q_{k}-A_{k}\right| A_{0} \mid \leqq B_{5} N^{-\frac{3}{2}}$. It follows that

$$
\left|\tilde{\rho}(t, p)-\frac{\tilde{\nu}(t, p)}{\tilde{\nu}(0, p)}\right| \leqq B_{6} N^{-\frac{3}{2}} \exp \left\{-\frac{c t^{2}}{4}\right\}
$$

and combined with $(2.52)$ this yields

$$
\int_{N^{-2} \leqq|t| \leqq b N^{\frac{3}{2}}}\left|\frac{\rho(t, p)-\tilde{\rho}(t, p)}{t}\right| d t \leqq B_{7}\left(N^{-\frac{3}{2}} \log N+N^{-\frac{5}{4}}\right) \leqq B_{8} N^{-\frac{5}{4}} .
$$

Together with (2.50) this proves $(2.49)$ and the theorem.

Two remarks should be made with regard to Theorem 2.1. The first one concerns condition (2.46) that does not occur in the preceding lemmas. The meaning of this condition is perhaps obscured by the fact that we make it do some odd jobs in the proof for which it is not really needed. We use it to show that (2.7) is satisfied for $N \geqq N_{0}$, but (2.25) ensures that the number of indices $j$ with $p_{j}=0\left(\right.$ or $\left.p_{j}=1\right)$ cannot exceed $m-C^{-1} c^{2} N+|\omega(p)| N^{\frac{1}{2}}\left(\right.$ or $n-C^{-1} c^{2} N+$ $\left.|\omega(p)| N^{\frac{1}{2}}\right)$ so that $|\omega(p)| \leqq C^{-1} c^{2} N^{\frac{1}{2}}$ already implies (2.7) for all $N$. Condition (2.46) is also used to obtain (2.50), but in (2.50) we may replace $N^{-2}$ by an arbitrarily high power of $N^{-1}$ without doing any damage to the proof, and then the trivial bound $|\omega(p)| \leqq N^{\frac{1}{2}}$ suffices. Finally we note that since

$$
\min (\lambda, 1-\lambda) \geqq \sigma^{2}(p)-N^{-\frac{1}{2}}|\omega(p)|,
$$

(2.46) forces $\lambda$ to be bounded away from 0 and 1 for large $N$, which is obviously important although it does not show up explicitly in the proof. However, here $|\omega(p)| \leqq \frac{1}{2} C^{-1} c^{2} N^{\frac{1}{2}}$ would be sufficient.

The basic function of assumption (2.46), however, is to avoid a large (or intermediate) deviation situation that the condition $\sum W_{j}=n$ would get us into if $\omega(p)=N^{-\frac{1}{2}}\left(E \sum W_{j}-n\right)$ would not be bounded. Technically speaking this is reflected in the proof at the point where $(2.46)$ is used to show that $\nu(0, p)$ is bounded away from zero. Also (2.46) ensures that (2.45) provides an expansion in powers of $N^{-\frac{1}{2}}$ to the required order.

To see what happens when condition $(2.46)$ is relaxed, we prefer not to try to 
adapt the proof of Theorem 2.1 but to answer this question more directly by remarking that the conditional distribution of $\sum a_{j} W_{j}$ given $\sum W_{j}=n$ remains unchanged if we replace $p$ by $\tilde{p}$ where $\tilde{p}_{j} /\left(1-\tilde{p}_{j}\right)=\xi p_{j} /\left(1-p_{j}\right) 1 \leqq j \leqq N$, for some $0 \leqq \xi \leqq \infty$. If $(2.7)$ is satisfied there exists a unique $\xi$ for which $\sum \tilde{p}_{j}=N \lambda$. Since $\omega(\tilde{p})=0$ it follows that if (2.21) and (2.22) are satisfied with $p$ replaced by $\tilde{p}$, then $(2.47)$ holds with $\tilde{R}(x, \tilde{p})$ instead of $\tilde{R}(x, p)$. Of course the snag is that in general $\tilde{p}$ can only be expressed analytically in terms of $p$ as an infinite series. However, if $\omega(p)=O\left(N^{\alpha}\right)$ for some $\alpha<\frac{1}{2}$, then a finite number of terms of this series will yield the required degree of accuracy and an explicit expansion for $R(x, p)$ can be obtained. If $\alpha=0$ this is expansion (2.45) but for $0<\alpha<\frac{1}{2}$ more terms have to be included.

The second remark concerns the remainder $O\left(N^{-\frac{5}{4}}\right)$ of our expansion. It is clear that by requiring that $\sum\left|a_{j}\right|^{5} \leqq C N$ in Theorem 2.1 one obtains $|R-\tilde{R}| \leqq$ $B N^{-\frac{3}{2}} \log (N+1)$. Of course the "natural" order of the remainder is $O\left(N^{-\frac{3}{2}}\right)$ and the factor $\log (N+1)$ is due only to technical difficulties in finding the conditional expectation of $\sum a_{j} W_{j}$ given $\sum W_{j}=n$.

The special case $p_{j}=\lambda, 1 \leqq j \leqq N$, which is random sampling without replacement, is worth singling out because it corresponds to the null-hypothesis in the two-sample problem. Let $\bar{\lambda}$ denote the vector $(\lambda, \ldots, \lambda)$. For $p=\bar{\lambda}$, (2.45) simplifies to

$$
\begin{aligned}
\tilde{R}(x, \bar{\lambda})=\Phi & \left(\frac{x}{\tau(\bar{\lambda})}\right)-\frac{\phi(x / \tau(\bar{\lambda}))}{\lambda(1-\lambda)}\left[\frac{\lambda(1-\lambda)}{2 N} H_{1}\left(\frac{x}{\tau(\bar{\lambda})}\right)\right. \\
& +\frac{\{\lambda(1-\lambda)\}^{\frac{1}{2}}(1-2 \lambda)}{6} \frac{\sum\left(a_{j}-a_{\bullet}\right)^{3}}{\left\{\sum\left(a_{j}-a_{\bullet}\right)^{2}\right\}^{\frac{3}{2}}} H_{2}\left(\frac{x}{\tau(\bar{\lambda})}\right) \\
& +\left\{\frac{1-6 \lambda+6 \lambda^{2}}{24} \frac{\sum\left(a_{j}-a_{\bullet}\right)^{4}}{\left\{\sum\left(a_{j}-a_{\bullet}\right)^{2}\right\}^{2}}-\frac{(1-2 \lambda)^{2}}{8 N}\right\} H_{3}\left(\frac{x}{\tau(\bar{\lambda})}\right) \\
& \left.+\frac{(1-2 \lambda)^{2}}{72} \frac{\left\{\sum\left(a_{j}-a_{\bullet}\right)^{3}\right\}^{2}}{\left\{\sum\left(a_{j}-a_{\bullet}\right)^{2}\right\}^{3}} H_{5}\left(\frac{x}{\tau(\bar{\lambda})}\right)\right],
\end{aligned}
$$

where

$$
\begin{gathered}
\tau^{2}(\bar{\lambda})=\frac{\lambda(1-\lambda)}{N} \sum_{j=1}^{N}\left(a_{j}-a_{\bullet}\right)^{2}, \\
a_{\bullet}=\bar{a}(\tilde{\lambda})=\frac{1}{N} \sum_{j=1}^{N} a_{j} .
\end{gathered}
$$

Define, with $l$ denoting Lebesgue measure on $R^{1}$,

$$
\gamma(\zeta)=l\left\{x: \exists j\left|x-a_{j}\right|<\zeta\right\} .
$$

For $p=\bar{\lambda}$, Theorem 2.1 yields

COROllary 2.1. Suppose that positive numbers $c, C, \delta$ and $\varepsilon$ exist such that

$$
\begin{gathered}
\varepsilon \leqq \lambda \leqq 1-\varepsilon, \\
\frac{1}{N} \sum_{j=1}^{N}\left(a_{j}-a_{\bullet}\right)^{2} \geqq c, \quad \frac{1}{N} \sum_{j=1}^{N} a_{j}{ }^{4} \leqq C,
\end{gathered}
$$




$$
\gamma(\zeta) \geqq \delta N \zeta \quad \text { for some } \zeta \geqq N^{-\frac{3}{2}} \log N .
$$

Then there exists $B>0$ depending only on $c, C, \delta$ and $\varepsilon$ such that

$$
\sup _{x}|R(x, \bar{\lambda})-\tilde{R}(x, \bar{\lambda})| \leqq B N^{-\S} .
$$

Note that there is considerable further simplification in (2.56) if we either have almost equal sample sizes, i.e., $\lambda=\frac{1}{2}+O\left(N^{-\frac{3}{4}}\right)$, or antisymmetric scores, i.e., $a_{j}+a_{N-j+1}$ is constant for all $j$. The latter happens for the locally most powerful rank test against shift alternatives when the underlying distribution is symmetric. In either case the $H_{2}$ and $H_{5}$ terms disappear so that the correction to the leading normal term is of order $N^{-1}$ only and is due solely to a correction to the variance, the $H_{1}$ term, and a kurtosis correction corresponding to $H_{3}$.

3. An unconditional expansion. We encounter several difficulties on the way to a usable unconditional expansion:

(i) the distribution of $Z$ is awkward to handle analytically;

(ii) as in ABZ (1976), the random variables obtained by substituting $P$ for $p$ in $\tilde{\rho}$ or $\tilde{R}$ are generally not summable;

(iii) again as in ABZ (1976), final simplification is not possible with our present techniques unless we assume that the sequence of alternatives is contiguous to the hypothesis as $N \rightarrow \infty$.

In this section we shall deal with the first two difficulties. Although we do not assume contiguity we shall be governed in the form of our expansion, which will involve polynomials in $\left(P_{j}-\lambda\right)$, in the number of terms that we calculate and in what we relegate to the remainder by the consideration that we expect $P_{j}=\lambda+O_{P}\left(N^{-\frac{1}{2}}\right)$ and $\sum\left(P_{j}-\lambda\right)=O_{P}(1)$.

Recall that we assumed that $X_{1}, \ldots, X_{N}$ are independent, $X_{1}, \ldots, X_{m}$ having common density $f$ and $X_{m+1}, \cdots, X_{N}$ having density $g$. We shall write $P$ for probabilities and $E$ for expectations calculated under this model. In addition we need to consider an auxiliary model where $X_{1}, \ldots, X_{N}$ are i.i.d. with common density $h=(1-\lambda) f+\lambda g$ and df $H=(1-\lambda) F+\lambda G$. We shall write $P_{H}$ for probabilities, $E_{H}$ for expectations and $\sigma_{H}{ }^{2}$ for variances calculated under this second model.

To simplify our notation we assume from this point on that

$$
\sum_{j=1}^{N} a_{j}=0 \text {. }
$$

Since $T=\sum\left(a_{j}-a_{0}\right) V_{j}+n a_{0}$ it is obvious how all expansions need to be modified if (3.1) does not hold.

We meet difficulty (i) through

Lemma 3.1 .

$$
E \exp \left\{i t N^{-\frac{1}{2}} T\right\}=\frac{E_{H} \nu(t, P) \exp \left\{i t N^{-\frac{1}{2}} \sum a_{j} P_{j}\right\}}{2 \pi N^{\frac{1}{2}} B_{N, n}(\lambda)},
$$


where

$$
B_{N, n}(\lambda)=\left(\begin{array}{l}
N \\
n
\end{array}\right) \lambda^{n}(1-\lambda)^{N-n} .
$$

Proof. Under our original model the density of $Z$ at the point $z=\left(z_{1}, \cdots, z_{N}\right)$ with $z_{1}<z_{2}<\cdots<z_{N}$ is given by

$$
\sum \prod_{j=1}^{m} f\left(z_{i_{j}}\right) \prod_{j=m+1}^{N} g\left(z_{i_{j}}\right),
$$

where the sum ranges over all permutations $i_{1}, \ldots, i_{N}$ of $1, \ldots, N$. Under our second model this density is

$$
N ! \prod_{j=1}^{N}\left[(1-\lambda) f\left(z_{j}\right)+\lambda g\left(z_{j}\right)\right] .
$$

By the Radon-Nikodym theorem and Lemma 2.1,

$$
\begin{aligned}
E \exp \left\{i t N^{-\frac{1}{2}} T\right\} & =E \frac{\nu(t, P)}{\nu(0, P)} \exp \left\{i t N^{-\frac{1}{2}} \sum a_{j} P_{j}\right\} \\
& =E_{H} \frac{\nu(t, P)}{\nu(0, P)} \exp \left\{i t N^{-\frac{1}{2}} \sum a_{j} P_{j}\right\} \sum \prod_{j=1}^{m} \frac{f\left(Z_{i_{j}}\right)}{h\left(Z_{i_{j}}\right)} \prod_{j=m+1}^{N} \frac{g\left(Z_{i_{j}}\right)}{h\left(Z_{i_{j}}\right)} \frac{1}{N !} \\
& =\left[B_{N, n}(\lambda)\right]^{-1} E_{H} \frac{\nu(t, P)}{\nu(0, P)} \exp \left\{i t N^{-\frac{1}{2}} \sum a_{j} P_{j}\right\} c(P),
\end{aligned}
$$

where $c$ is defined by (2.6) or (2.12). The lemma follows from (2.11) and (2.13).

Lemma 3.1 shows that we are concerned with $\tilde{\nu}$ rather than $\tilde{\rho}$, but since $\tilde{\nu}$ as a function of $P$ is no more summable than $\tilde{\rho}$, we still have to face difficulty (ii). We do this by showing that $\tilde{\nu}$ may be replaced by a summable function $\nu^{*}$ outside a set that will later be seen to have sufficiently small probability. Define

$$
\nu^{*}(t, p)=\left[\frac{2 \pi}{\lambda(1-\lambda)}\right]^{\frac{1}{2}} \exp \left\{-\frac{\lambda(1-\lambda)}{2 N} \sum a_{j}{ }^{2} t^{2}\right\} \sum_{k=0}^{6} A_{k}{ }^{*}(p)(i t)^{k},
$$

where

$$
\begin{aligned}
A_{0}^{*}(p)=1 & +\frac{1}{2 \lambda(1-\lambda) N}\left[\sum\left(p_{j}-\lambda\right)^{2}-\left\{\sum\left(p_{j}-\lambda\right)\right\}^{2}\right. \\
& \left.-\frac{1-\lambda+\lambda^{2}}{6}\right], \\
A_{1}^{*}(p)=N^{-\frac{3}{2}} \sum a_{j} p_{j}\left[1-\frac{1-2 \lambda}{\lambda(1-\lambda)} \sum\left(p_{j}-\lambda\right)\right] & \\
A_{2}{ }^{*}(p)=\frac{(1-2 \lambda)}{2 N} \sum a_{j}{ }^{2}\left(p_{j}-\lambda\right)-\frac{\sum a_{j}{ }^{2}}{2 N^{2}} & \quad \times\left[(1-2 \lambda) \sum\left(p_{j}-\lambda\right)-\lambda(1-\lambda)\right] \\
& -\frac{1}{2 N} \sum a_{j}{ }^{2}\left(p_{j}-\lambda\right)^{2}-\frac{(1-2 \lambda)^{2}}{2 \lambda(1-\lambda) N^{2}}\left\{\sum a_{j} p_{j}\right\}^{2}, \\
& \frac{N^{-\frac{3}{2}}}{6}\left[\lambda(1-\lambda)(1-2 \lambda) \sum a_{j}{ }^{3}+\left(1-6 \lambda+6 \lambda^{2}\right) \sum a_{j}{ }^{3}\left(p_{j}-\lambda\right)\right. \\
& \left.-\frac{3}{N}(1-2 \lambda)^{2} \sum a_{j}{ }^{2} \sum a_{j} p_{j}\right]
\end{aligned}
$$




$$
\begin{aligned}
A_{4}{ }^{*}(p)= & \frac{\lambda(1-\lambda)\left(1-6 \lambda+6 \lambda^{2}\right)}{24 N^{2}} \sum a_{j}{ }^{4}-\frac{\lambda(1-\lambda)(1-2 \lambda)^{2}}{8 N^{3}}\left\{\sum a_{j}{ }^{2}\right\}^{2} \\
& \quad+\frac{(1-2 \lambda)^{2}}{8 N^{2}}\left\{\sum a_{j}{ }^{2}\left(p_{j}-\lambda\right)\right\}^{2} \\
A_{5}{ }^{*}(p)= & \frac{N^{-\frac{5}{2}}}{12} \lambda(1-\lambda)(1-2 \lambda)^{2} \sum a_{j}{ }^{3} \sum a_{j}{ }^{2}\left(p_{j}-\lambda\right) \\
A_{6}{ }^{*}(p)= & \frac{\lambda^{2}(1-\lambda)^{2}(1-2 \lambda)^{2}}{72 N^{3}}\left\{\sum a_{j}{ }^{3}\right\}^{2} .
\end{aligned}
$$

Lemma 3.2. Suppose that (3.1) holds and positive numbers $c, C$ and $\varepsilon$ exist such that (2.21) is satisfied and

$$
\varepsilon \leqq \lambda \leqq 1-\varepsilon
$$

Then there exist positive numbers $B$ and $\beta$ depending only on $c, C$ and $\varepsilon$ such that

$$
\begin{aligned}
\left|\tilde{\nu}(t, p)-\nu^{*}(t, p)\right| \leqq B & \exp \left\{-\beta t^{2}\right\}\left[\left\{N^{-\frac{3}{2}}+N^{-\frac{5}{4}}|t|\right\}\left\{1+N \sum\left(p_{j}-\lambda\right)^{4}\right\}\right. \\
& \left.+N^{-\frac{3}{2}}\left\{\sum\left(p_{j}-\lambda\right)^{4}\right\}\right] .
\end{aligned}
$$

Proof. For simplicity we make use of order symbols in this proof and $O(x)$ will denote a quantity that is bounded by $B_{1}|x|$ where $B_{1}$ depends only on $c, C$ and $\varepsilon$.

Suppose first that $|\omega(p)|>1$. Then (2.21) and (3.5) are easily seen to imply that $\left|\nu^{*}(t, p)\right|=O\left(\omega^{2}(p) \exp \left\{-\varepsilon(1-\varepsilon) c t^{2} / 4\right\}\right)$, whereas for $\tilde{\nu}(t, p)$ we have the bound (2.51). The right-hand side of (3.6), however, contains a term $B N^{\frac{1}{2}} \omega^{4}(p) \exp \left\{-\beta t^{2}\right\}$ so that the lemma is trivial for $|\omega(p)|>1$.

We therefore assume that $|\omega(p)| \leqq 1$. Noting that $\sigma^{2}(p)$ is bounded away from zero (cf. (2.25)), we expand $\sigma^{-2}, \bar{a}, \tau^{2}$ and $\kappa_{r, i}$ about the point $p_{j}=\lambda$, $1 \leqq j \leqq N$, using elementary inequalities to bound the remainders in terms of $N$ and

$$
M_{1}=N^{-1} \sum\left(p_{j}-\lambda\right)^{4}, \quad M_{2}=N^{-1}\left|\sum\left(p_{j}-\lambda\right)\right| .
$$

We find

$$
\begin{aligned}
\frac{1}{\sigma^{2}(p)}= & \frac{1}{\lambda(1-\lambda)}\left[1-\frac{(1-2 \lambda)}{\lambda(1-\lambda) N} \sum\left(p_{j}-\lambda\right)+\frac{1}{\lambda(1-\lambda) N} \sum\left(p_{j}-\lambda\right)^{2}\right] \\
& +O\left(M_{1}+M_{2}^{2}\right) \\
= & \frac{1}{\lambda(1-\lambda)}+O\left(M_{1}^{\frac{1}{2}}+M_{2}\right), \\
& \bar{a}(p)=\frac{(1-2 \lambda)}{\lambda(1-\lambda) N} \sum a_{j} p_{j}+O\left(M_{1}^{\frac{1}{2}}\right)=O\left(M_{1}^{\frac{1}{4}}\right), \\
\tau^{2}(p)= & \frac{\lambda(1-\lambda)}{N} \sum a_{j}{ }^{2}+\frac{(1-2 \lambda)}{N} \sum a_{j}{ }^{2}\left(p_{j}-\lambda\right)-\frac{1}{N} \sum a_{j}{ }^{2}\left(p_{j}-\lambda\right)^{2} \\
& \quad-\frac{(1-2 \lambda)^{2}}{\lambda(1-\lambda) N^{2}}\left\{\sum a_{j} p_{j}\right\}^{2}+O\left(M_{1}^{\frac{3}{4}}\right),
\end{aligned}
$$




$$
\begin{aligned}
& \kappa_{3,0}(p)=\lambda(1-\lambda)(1-2 \lambda)+O\left(M_{1}^{\frac{1}{2}}+M_{2}\right), \\
& \kappa_{3,1}(p)=-\frac{2 \lambda(1-\lambda)}{N} \sum a_{j} p_{j}+O\left(M_{1}^{\frac{1}{2}}\right), \\
& \kappa_{3,2}(p)=\frac{\lambda(1-\lambda)(1-2 \lambda)}{N} \sum a_{j}^{2}+O\left(M_{1}^{\frac{1}{4}}\right), \\
& \kappa_{3,3}(p)=\frac{\lambda(1-\lambda)(1-2 \lambda)}{N} \sum a_{j}{ }^{3}+\frac{\left(1-6 \lambda+6 \lambda^{2}\right)}{N} \sum a_{j}{ }^{3}\left(p_{j}-\lambda\right) \\
& -\frac{3(1-2 \lambda)^{2}}{N^{2}} \sum a_{j}{ }^{2} \sum a_{j} p_{j}+O\left(N^{\frac{1}{4}} M_{1}^{\frac{1}{2}}\right) \quad(=O(1)), \\
& \kappa_{4,0}(p)=\lambda(1-\lambda)\left(1-6 \lambda+6 \lambda^{2}\right)+O\left(M_{1}^{\frac{1}{4}}\right), \\
& \kappa_{4,1}(p)=O\left(M_{1}^{\frac{1}{4}}\right) \text {, } \\
& \kappa_{4,2}(p)=\frac{\lambda(1-\lambda)(1-6 \lambda+6 \lambda)^{2}}{N} \sum a_{j}{ }^{2}+O\left(M_{1}^{\frac{1}{4}}\right), \\
& \kappa_{4,3}(p)=O(1) \text {, } \\
& \kappa_{4,4}(p)=\frac{\lambda(1-\lambda)\left(1-6 \lambda+6 \lambda^{2}\right)}{N} \sum a_{j}{ }^{4}+O\left(N^{\frac{1}{4}} M_{1^{\frac{1}{4}}}\right) \quad(=O(1)) .
\end{aligned}
$$

To illustrate computations involved we present the argument for $\kappa_{3.3}$. By $(2.21)$, the result for $\bar{a}(p)$ and the fact that $0 \leqq M_{1} \leqq 1$, we have

$$
\begin{aligned}
\kappa_{3,3}(p)=N^{-1} & \sum p_{j}\left(1-p_{j}\right)\left(1-2 p_{j}\right) a_{j}{ }^{3} \\
& \quad-3 N^{-1} \bar{a}(p) \sum p_{j}\left(1-p_{j}\right)\left(1-2 p_{j}\right) a_{j}{ }^{2}+O\left(M_{1}^{\frac{1}{2}}\right) \\
= & N^{-1} \lambda(1-\lambda)(1-2 \lambda) \sum a_{j}{ }^{3}+N^{-1}\left(1-6 \lambda+6 \lambda^{2}\right) \sum a_{j}{ }^{3}\left(p_{j}-\lambda\right) \\
& \quad-3 N^{-2}(1-2 \lambda)^{2} \sum a_{j}{ }^{2} \sum a_{j} p_{j} \\
& +O\left(M_{1}^{\frac{1}{2}}+N^{-1} \sum\left|a_{j}\right|^{3}\left(p_{j}-\lambda\right)^{2}+N^{-1} M_{1}^{\frac{1}{4}} \sum a_{j}{ }^{2}\left|p_{j}-\lambda\right|\right) .
\end{aligned}
$$

Hölder's inequality and (2.21) imply that

$$
\begin{aligned}
& N^{-1} M_{1}^{\frac{1}{4}} \sum a_{j}{ }^{2}\left|p_{j}-\lambda\right| \leqq N^{-1} M_{1}^{\frac{1}{4}}\left(\sum\left|a_{j}\right|^{\frac{8}{3}}\right)^{\frac{3}{4}}\left(N M_{1}\right)^{\frac{1}{4}}=O\left(M_{1}^{\frac{1}{2}}\right), \\
& N^{-1} \sum\left|a_{j}\right|^{3}\left(p_{j}-\lambda\right)^{2} \leqq N^{-1}(C N)^{\frac{1}{4}} \sum a_{j}{ }^{2}\left(p_{j}-\lambda\right)^{2} \leqq C^{\frac{1}{4}} N^{-\frac{3}{4}}\left(N M_{1} \sum a_{j}{ }^{4}\right)^{\frac{1}{2}} \\
&=O\left(N^{\frac{1}{4}} M^{\frac{1}{2}}\right) .
\end{aligned}
$$

As $\bar{a}(p)$ is bounded, $\kappa_{3,3}(p)$ is obviously also $O(1)$. Note that the atypical order of the remainder $O\left(N^{\frac{1}{4}} M_{1}^{\frac{1}{2}}\right)$ originates from the term $O\left(N^{-1} \sum\left|a_{j}\right|^{3}\left(p_{j}-\lambda\right)^{2}\right)$ where we have to sacrifice a factor $O\left(N^{-\frac{1}{4}}\right)$ in order to apply Hölder's inequality and (2.21). The same thing occurs for $\kappa_{4,4}(p)$.

For $\mu_{k}(p)$ defined by (2.28) we find

$$
\begin{aligned}
& \mu_{1}(p)=\frac{1}{\lambda(1-\lambda) N^{\frac{1}{2}}} \sum\left(p_{j}-\lambda\right)+O\left(M_{1}^{\frac{1}{2}}+N^{\frac{1}{2}} M_{2}^{2}\right) \\
& \mu_{2}(p)=\frac{1}{\lambda(1-\lambda)}+O\left(M_{1}^{\frac{1}{2}}+M_{2}+N M_{2}^{2}\right)
\end{aligned}
$$




$$
\begin{aligned}
& \mu_{3}(p)=\frac{3}{\lambda^{2}(1-\lambda)^{2} N^{\frac{1}{2}}} \sum\left(p_{j}-\lambda\right)+O\left(M_{1}^{\frac{1}{2}}+N M_{2}^{2}\right) \\
& \mu_{4}(p)=\frac{3}{\lambda^{2}(1-\lambda)^{2}}+O\left(M_{1}^{\frac{1}{2}}+M_{2}+N M_{2}^{2}\right) \\
& \mu_{5}(p)=O\left(N^{\frac{1}{2}} M_{2}\right) \\
& \mu_{6}(p)=\frac{15}{\lambda^{3}(1-\lambda)^{3}}+O\left(M_{1}^{\frac{1}{2}}+M_{2}+N M_{2}^{2}\right) .
\end{aligned}
$$

Straightforward but tedious calculation now yields

$$
\begin{aligned}
\sum_{k=0}^{6} A_{k}(p)(i t)^{k} & =\left[1+\frac{(1-2 \lambda)}{2 \lambda(1-\lambda) N} \sum\left(p_{j}-\lambda\right)-\frac{\left(1-\lambda+\lambda^{2}\right)}{12 \lambda(1-\lambda) N]}\right. \\
= & +\frac{\sum a_{j} p_{j}}{N^{\frac{3}{2}}} i t-\frac{\sum a_{j}{ }^{2}}{2 N^{2}}\left[(1-2 \lambda) \sum\left(p_{j}-\lambda\right)-\lambda(1-\lambda)\right](i t)^{2} \\
& +\frac{1}{6 N^{\frac{3}{2}}}\left[\lambda(1-\lambda)(1-2 \lambda) \sum a_{j}{ }^{3}+\left(1-6 \lambda+6 \lambda^{2}\right) \sum a_{j}{ }^{3}\left(p_{j}-\lambda\right)\right. \\
& \left.-\frac{3(1-2 \lambda)^{2}}{N} \sum a_{j}{ }^{2} \sum a_{j} p_{j}\right](i t)^{3} \\
& +\frac{\lambda(1-\lambda)}{24 N^{2}}\left[\left(1-6 \lambda+6 \lambda^{2}\right) \sum a_{j}{ }^{4}-\frac{3(1-2 \lambda)^{2}}{N}\left\{\sum a_{j}{ }^{2}\right\}^{2}\right](i t)^{4} \\
& +\frac{\lambda^{2}(1-\lambda)^{2}(1-2 \lambda)^{2}}{72 N^{3}}\left\{\sum a_{j}{ }^{3}\right\}^{2}(i t)^{6}+O\left(\left(|t|^{3}+t^{4}\right)\left[N^{-\frac{5}{4}}+N^{-\frac{1}{4}} M_{1}{ }^{\frac{1}{2}}\right]\right. \\
& \left.+\left(1+t^{6}\right)\left[N^{-\frac{3}{2}}+N^{-\frac{1}{2}} M_{1}{ }^{\frac{1}{2}}+N^{\frac{1}{2}} M_{2}{ }^{2}\right]\right) .
\end{aligned}
$$

Next we expand the remaining factor in (2.30). Because both $\tau^{2}(p)$ and its leading term $\lambda(1-\lambda) N^{-1} \sum a_{j}{ }^{2}$ are bounded away from zero, there exists $\beta>0$ depending only on $c, C$ and $D$, such that

$$
\begin{aligned}
& \frac{(2 \pi)^{\frac{1}{2}}}{\sigma(p)} \exp \left\{-\frac{\omega^{2}(p)}{2 \sigma^{2}(p)}-\frac{\tau^{2}(p) t^{2}}{2}-i \omega(p) \bar{a}(p) t\right\} \\
&=\left[\frac{2 \pi}{\lambda(1-\lambda)}\right]^{\frac{1}{2}} \exp \left\{-\frac{\lambda(1-\lambda)}{2 N} \sum a_{j}{ }^{2} t^{2}\right\} \\
& \quad \times\left[1-\frac{1}{2 \lambda(1-\lambda) N}\left\{(1-2 \lambda) \sum\left(p_{j}-\lambda\right)-\sum\left(p_{j}-\lambda\right)^{2}+\left\{\sum\left(p_{j}-\lambda\right)\right\}^{2}\right\}\right. \\
&-\frac{(1-2 \lambda)}{\lambda(1-\lambda) N^{\frac{3}{2}}} \sum\left(p_{j}-\lambda\right) \sum a_{j} p_{j}(i t)+\frac{1}{2 N}\left\{(1-2 \lambda) \sum a_{j}{ }^{2}\left(p_{j}-\lambda\right)\right. \\
&\left.-\sum a_{j}{ }^{2}\left(p_{j}-\lambda\right)^{2}-\frac{(1-2 \lambda)^{2}}{\lambda(1-\lambda) N}\left\{\sum a_{j} p_{j}\right\}^{2}\right\}(i t)^{2} \\
&\left.+\frac{(1-2 \lambda)^{2}}{8 N^{2}}\left\{\sum a_{j}{ }^{2}\left(p_{j}-\lambda\right)\right\}^{2}(i t)^{4}\right] \\
&+O\left(\exp \left\{-\beta t^{2}\right\}\left[N^{-\frac{3}{2}}+N^{\frac{1}{2}} M_{1}+N^{\frac{5}{2}} M_{2}{ }^{4}\right]\right) .
\end{aligned}
$$

Multiplication by (3.7) yields (3.6). 
Here is our first unconditional expansion. Define

$$
\begin{gathered}
\rho(t)=E \exp \left\{i t N^{-\frac{1}{2}} T\right\}, \\
\rho^{*}(t)=\exp \left\{-\frac{\lambda(1-\lambda)}{2 N} \sum a_{j}{ }^{2} t^{2}\right\} E_{H}\left[\exp \left\{i t N^{-\frac{1}{2}} \sum a_{j} P_{j}\right\}\right. \\
\times\left\{1+\frac{1}{2 \lambda(1-\lambda) N}\left(\sum\left(P_{j}-\lambda\right)^{2}-\left\{\sum\left(P_{j}-\lambda\right)\right\}^{2}\right)\right. \\
\left.\left.+\sum_{k=1}^{6} A_{k}^{*}(P)(i t)^{k}\right\}\right] .
\end{gathered}
$$

Lemma 3.3. Suppose that (3.1) holds and that positive numbers $c, C, \delta, \delta^{\prime}$ and $\varepsilon$ exist with $\delta^{\prime}<\min \left(\frac{1}{2}, \delta / 2, c^{2} C^{-1} / 4\right)$ and such that $(2.62)$ is satisfied and

$$
\begin{gathered}
\frac{1}{N} \sum a_{j}{ }^{2} \geqq c, \quad \frac{1}{N} \sum a_{j}{ }^{4} \leqq C, \\
P_{H}\left(\varepsilon \leqq \frac{\lambda g\left(X_{1}\right)}{h\left(X_{1}\right)} \leqq 1-\varepsilon\right) \geqq 1-\delta^{\prime} .
\end{gathered}
$$

Then there exist positive numbers $b, B, \beta_{1}$ and $\beta_{2}$ depending only on $c, C, \delta, \delta^{\prime}$ and $\varepsilon$ such that for $|t| \leqq b N^{\frac{3}{2}}$,

$$
\begin{aligned}
\left|\rho(t)-\rho^{*}(t)\right| \leqq B & {\left[\exp \left\{-\beta_{1} t^{2}\right\}\left(N^{-\frac{3}{2}}+N^{-\frac{5}{4}}|t|\right)\right.} \\
& \left.\times\left\{1+N^{2} E_{H}\left(\frac{g\left(X_{1}\right)}{h\left(X_{1}\right)}-1\right)^{4}\right\}+N^{-\beta_{2} \log N}\right] .
\end{aligned}
$$

Proof. In this proof we again use $O$ symbols that are uniform for fixed $c, C$, $\delta, \delta^{\prime}$ and $\varepsilon$. Note that $E_{H}\left\{g\left(X_{1}\right) / h\left(X_{1}\right)\right\}=1$, so that (3.11) and Markov's inequality ensure that $\min (\lambda, 1-\lambda) \geqq \varepsilon\left(1-\delta^{\prime}\right)$.

Take a number $\delta^{\prime \prime} \in\left(\delta^{\prime}, \min \left(\frac{1}{2}, \delta / 2, c^{2} C^{-1} / 4\right)\right.$ and define the event $E$ by

$$
\begin{aligned}
E & =\left\{\varepsilon \leqq P_{j} \leqq 1-\varepsilon \text { for at least }\left(1-\delta^{\prime \prime}\right) N \text { indices } j\right\} \\
& =\left\{\varepsilon \leqq \frac{\lambda g\left(X_{j}\right)}{h\left(X_{j}\right)} \leqq 1-\varepsilon \text { for at least }\left(1-\delta^{\prime \prime}\right) N \text { indices } j\right\}
\end{aligned}
$$

Applying an exponential bound for binomial probabilities (cf. Okamoto (1958)) we find that (3.11) implies

$$
P_{H}(E) \geqq 1-\exp \left\{-2 N\left(\delta^{\prime \prime}-\delta^{\prime}\right)^{2}\right\} .
$$

Because $\lambda$ and $(1-\lambda)$ are bounded away from 0 , the same is true for $N^{\frac{1}{2}} B_{N, n}(\lambda)$. Also, (2.10) and (2.11) imply that $|\nu(t, p)| \leqq 2 \pi N^{\frac{1}{2}}$ for all $t$ and $p$. Hence application of Lemma 3.1 shows that

$$
\rho(t)=\frac{E_{H} \nu(t, P) \exp \left\{i t N^{-\frac{1}{2}} \sum a_{j} P_{j}\right\} \chi_{E}}{2 \pi N^{\frac{1}{2}} B_{N, n}(\lambda)}+O\left(\exp \left\{-N\left(\delta^{\prime \prime}-\delta^{\prime}\right)^{2}\right\}\right),
$$

where $\chi_{E}$ denotes the indicator of $E$. 
Since $\delta^{\prime \prime}<\delta / 2,(2.62)$ ensures the validity of (2.22) on the set $E$ with $\delta$ replaced by $\delta-2 \delta^{\prime \prime}$. If $\Sigma^{\prime}$ denotes summation over those indices $j$ for which $P_{j} \notin[\varepsilon, 1-\varepsilon]$ and $k$ denotes the number of these indices, then $k \leqq \delta^{\prime \prime} N$ on $E$ and as a result

$$
\begin{aligned}
\tau^{2}(P) & \geqq \frac{\varepsilon(1-\varepsilon)}{N}\left[\sum_{j=1}^{N}\left(a_{j}-\bar{a}(P)\right)^{2}-\Sigma^{\prime}\left(a_{j}-\bar{a}(P)\right)^{2}\right] \\
& \geqq \frac{\varepsilon(1-\varepsilon)}{N}\left[\sum_{j=1}^{N} a_{j}{ }^{2}+N\{\bar{a}(P)\}^{2}-2 \sum^{\prime} a_{j}{ }^{2}-2 k\{\bar{a}(P)\}^{2}\right] \\
& \geqq \frac{\varepsilon(1-\varepsilon)}{N}\left[c N-2\left\{k \sum^{\prime} a_{j}^{4}\right\}^{\frac{1}{2}}\right] \geqq \varepsilon(1-\varepsilon)\left[c-2\left\{\delta^{\prime \prime} C\right\}^{\frac{1}{2}}\right]>0
\end{aligned}
$$

on $E$, because $\delta^{\prime \prime}<\min \left(\frac{1}{2}, c^{2} C^{-1} / 4\right)$.

We have shown that on the set $E, a$ and $P$ satisfy the conditions on $a$ and $p$ in Lemmas 2.3 and 3.2. Combining (3.14), (2.31) and (3.6) we obtain

$$
\begin{aligned}
\rho(t)= & \frac{E_{H} \nu^{*}(t, P) \exp \left\{i t N^{-\frac{1}{2}} \sum a_{j} P_{j}\right\} \chi_{E}}{2 \pi N^{\frac{1}{2}} B_{N, n}(\lambda)} \\
& +O\left(N^{-\beta_{2} \log N}+\exp \left\{-\beta_{1} t^{2}\right\}\left[\left\{N^{-\frac{3}{2}}+N^{-\frac{\xi}{4}}|t|\right\}\right.\right. \\
& \left.\left.\times\left\{1+N E_{H} \sum\left(P_{j}-\lambda\right)^{4}\right\}+N^{-\frac{3}{2}} E_{H}\left\{\sum\left(P_{j}-\lambda\right)\right\}^{4}\right]\right)
\end{aligned}
$$

for $|t| \leqq b N^{\frac{3}{2}}$, where $b, \beta_{1}$ and $\beta_{2}$ depend on $c, C, \delta, \delta^{\prime}$ and $\varepsilon$ only.

Because of (3.13) and the fact that $\nu^{*}(t, p)=O(N),(3.15)$ remains valid if we delete $\chi_{E}$. Using

$$
2 \pi N^{\frac{1}{2}} B_{N, n}(\lambda)=\left[\frac{2 \pi}{\lambda(1-\lambda)}\right]^{\frac{1}{2}}\left(1-\frac{1-\lambda+\lambda^{2}}{12 \lambda(1-\lambda) N}\right)+O\left(N^{-2}\right)
$$

one easily verifies that in (3.15) the first term on the right may be replaced by $\rho^{*}(t)$ without changing the order of the remainder. Since

$$
\begin{gathered}
E_{H} \sum\left(P_{j}-\lambda\right)^{4}=E_{H} \sum\left(\frac{\lambda g\left(X_{i}\right)}{h\left(X_{i}\right)}-\lambda\right)^{4}=\lambda^{4} N E_{H}\left(\frac{g\left(X_{1}\right)}{h\left(X_{1}\right)}-1\right)^{4}, \\
E_{H}\left\{\sum\left(P_{j}-\lambda\right)\right\}^{4}=\lambda^{4} E_{H}\left\{\sum\left(\frac{g\left(X_{i}\right)}{h\left(X_{i}\right)}-1\right)\right\}^{4} \leqq 3 \lambda^{4} N^{2} E_{H}\left(\frac{g\left(X_{1}\right)}{h\left(X_{1}\right)}-1\right)^{4},
\end{gathered}
$$

the proof of the lemma is complete.

Define

$$
\pi_{j}=E_{H} P_{j}, \quad \pi=\left(\pi_{1}, \cdots, \pi_{x}\right) .
$$

In the remaining part of this section we obtain a further expansion for $\rho(t)$ and convert this expansion into one for the $\mathrm{df}$ of $T$. Although we still do not assume contiguity, we shall be guided in what terms we include in the remainder by the fact that under contiguous alternatives we expect $\left(P_{j}-\pi_{j}\right)$ to behave roughly like $O_{P_{H}}\left(N^{-1}\right)$. Let

$$
K(x)=\Phi(x)-\phi(x) \sum_{k=0}^{5} \alpha_{k} H_{k}(x)
$$


where $\Phi$ and $\phi$ denote the standard normal df and its density, the Hermite polynomials $H_{k}$ are given by (2.43) and

$$
\begin{aligned}
\alpha_{0}= & \frac{\sum a_{j} \pi_{j}}{\left\{\lambda(1-\lambda) \sum a_{j}{ }^{2}\right\}^{\frac{1}{2}} N}, \\
\alpha_{1}= & \frac{\sigma_{H}{ }^{2}\left(\sum a_{j} P_{j}\right)-\sum a_{j}{ }^{2} E_{H}\left(P_{j}-\lambda\right)^{2}+(1-2 \lambda) \sum a_{j}{ }^{2}\left(\pi_{j}-\lambda\right)}{2 \lambda(1-\lambda) \sum a_{j}{ }^{2}} \\
& \quad-\frac{(1-2 \lambda)^{2}\left\{\sum a_{j} \pi_{j}\right\}^{2}}{2 \lambda^{2}(1-\lambda)^{2} N \sum a_{j}{ }^{2}}+\frac{1}{2 N}, \\
\alpha_{2}= & \left(\left[\lambda(1-\lambda)(1-2 \lambda) \sum a_{j}{ }^{3}+\left(1-6 \lambda+6 \lambda^{2}\right) \sum a_{j}{ }^{3}\left(\pi_{j}-\lambda\right)\right.\right. \\
& \left.\left.\quad-3(1-2 \lambda)^{2} N^{-1} \sum a_{j}{ }^{2} \sum a_{j} \pi_{j}\right]\right) /\left(6\left\{\lambda(1-\lambda) \sum a_{j}{ }^{2}\right\}^{3}\right), \\
\alpha_{3}= & \left(\lambda(1-\lambda)\left(1-6 \lambda+6 \lambda^{2}\right) \sum a_{j}{ }^{4}-3 \lambda(1-\lambda)(1-2 \lambda)^{2} N^{-1}\left\{\sum a_{j}{ }^{2}\right\}^{2}\right. \\
& \left.\quad+3(1-2 \lambda)^{2}\left\{\sum a_{j}{ }^{2}\left(\pi_{j}-\lambda\right)\right\}^{2}\right) /\left(24\left\{\lambda(1-\lambda) \sum a_{j}{ }^{2}\right\}^{2}\right), \\
\alpha_{4}= & \frac{(1-2 \lambda)^{2} \sum a_{j}{ }^{3} \sum a_{j}{ }^{2}\left(\pi_{j}-\lambda\right)}{12\{\lambda(1-\lambda)\}^{\frac{3}{2}}\left\{\sum a_{j}{ }^{2}\right\}^{\frac{5}{2}}}, \\
\alpha_{5}= & \frac{(1-2 \lambda)^{2}\left\{\sum a_{j}{ }^{3}\right\}^{2}}{72 \lambda(1-\lambda)\left\{\sum a_{j}{ }^{2}{ }^{3}\right.} \cdot
\end{aligned}
$$

THEOREM 3.1. Suppose that (3.1) holds and that positive numbers $c, C, \delta$ and $\varepsilon$ exist such that (3.10) and (2.62) are satisfied and

$$
\varepsilon \leqq \lambda \leqq 1-\varepsilon
$$

Then there exists $B>0$ depending only on $c, C, \delta$ and $\varepsilon$ such that

$$
\begin{aligned}
\sup _{x} \mid & P\left(\frac{T}{\left\{\lambda(1-\lambda) \sum a_{j}^{2}\right\}^{\frac{1}{2}}} \leqq x\right)-K\left(x-\frac{\sum a_{j} \pi_{j}}{\left\{\lambda(1-\lambda) \sum a_{j}^{2}\right\}^{\frac{1}{2}}}\right) \mid \\
& \leqq B\left\{N^{-\frac{5}{4}}+N^{\frac{3}{3}} E_{H}\left(\frac{g\left(X_{1}\right)}{h\left(X_{1}\right)}-1\right)^{4}+N^{-\frac{1}{2}}\left[\sum\left\{E_{H}\left|P_{j}-\pi_{j}\right|^{3}\right\}^{\frac{9}{3}}\right]^{\frac{9}{3}}\right\} .
\end{aligned}
$$

Proof. In this proof $B_{i}$ and $\beta_{i}$ denote appropriately chosen positive numbers depending only on $c, C, \delta$ and $\varepsilon$. We shall have to consider the rv

$$
U=N^{-\frac{1}{2}} \sum a_{j}\left(P_{j}-\pi_{j}\right)
$$

and we note that

$$
\begin{aligned}
E_{H}|U|^{3} & \leqq N^{-\frac{3}{2}}\left[\sum\left|a_{j}\right|\left\{E_{H}\left|P_{j}-\pi_{j}\right|^{3}\right\}^{\frac{1}{3}}\right]^{3} \\
& \leqq C^{\frac{3}{4}} N^{-\frac{3}{4}}\left[\sum\left\{E_{H}\left|P_{j}-\pi_{j}\right|^{3}\right\}^{\frac{4}{5}}\right]^{\frac{9}{4}} .
\end{aligned}
$$

Since $\sup _{x}(1+|K(x)|) \leqq B_{1}\left(1+E_{H} U^{2}\right) \leqq B_{1}\left(2+E_{H}|U|^{3}\right)$ we may assume without loss of generality that $E_{H}|U|^{3} \leqq 1$, because otherwise (3.20) is satisfied trivially for $B=3 B_{1} C^{\frac{3}{3}}$. Hence $\sup _{x}(1+|K(x)|) \leqq 3 B_{1}$ and similar bounds $\left|\alpha_{k}\right| \leqq B_{2}\left(1+E_{H} U^{2}\right) \leqq 3 B_{2}$ and $\sup _{x}\left|K^{\prime}(x)\right| \leqq 3 B_{3}$ hold for $\alpha_{0}, \cdots, \alpha_{5}$ and for the derivative $K^{\prime}$ of $K$.

Take $\delta^{\prime}=\min \left(\frac{1}{4}, \delta / 4, c^{2} C^{-1} / 8\right)$. In view of $1+|K| \leqq 3 B_{1}$ it is again no loss of generality to assume that $E_{H}\left(g\left(X_{1}\right) / h\left(X_{1}\right)-1\right)^{4} \leqq \delta^{\prime} \varepsilon^{4} / 16$, because otherwise 
(3.22) with $B=48 B_{1} /\left(\delta^{\prime} \varepsilon^{4}\right)$ is trivially true. Hence by (3.19) and Markov's inequality

$$
P_{H}\left(\frac{1}{2} \varepsilon \leqq \frac{\lambda g\left(X_{1}\right)}{h\left(X_{1}\right)} \leqq 1-\frac{1}{2} \varepsilon\right) \geqq P_{H}\left(\left|\frac{g\left(X_{1}\right)}{h\left(X_{1}\right)}-1\right| \leqq \frac{1}{2} \varepsilon\right) \geqq 1-\delta^{\prime},
$$

so that the conditions of Lemma 3.3 are satisfied and (3.12) holds.

The proof hinges on the expansion

$$
\exp \left\{i t N^{-\frac{1}{2}} \sum a_{j} P_{j}\right\}=\exp \left\{i t N^{-\frac{1}{2}} \sum a_{j} \pi_{j}\right\}\left[1+i t U+\frac{1}{2}(i t U)^{2}\right]+O\left(|t U|^{3}\right)
$$

and its truncation to fewer terms. We apply this expansion to (3.9) and in the resulting expression we replace $P$ by $\pi$ wherever this is possible without giving rise to remainder terms that would be awkward to handle at this point. Using elementary inequalities to separate out and bound those parts of the remainder that depend on the $\left(P_{j}-\lambda\right)$ rather than on the $\left(P_{j}-\pi_{j}\right)$, we arrive at

$$
\begin{gathered}
\left|\rho^{*}(t)-\bar{\rho}(t)\right| \leqq B_{4}|t| \exp \left\{-\beta_{3} t^{2}\right\}\left[N^{-\frac{3}{2}}+N^{\frac{1}{2}} E_{H}\left(\frac{g\left(X_{1}\right)}{h\left(X_{1}\right)}-1\right)^{4}+E_{H}|U|^{3}\right. \\
\left.+N^{-1} E_{H}\left|U \sum a_{j}{ }^{2}\left(P_{j}-\pi_{j}\right)\right|+N^{-2} E_{H}\left\{\sum a_{j}{ }^{2}\left(P_{j}-\pi_{j}\right)\right\}^{2}\right], \\
\bar{\rho}(t)=\exp \left\{i t N^{-\frac{1}{2}} \sum a_{j} \pi_{j}-t^{2} \frac{\lambda(1-\lambda)}{2 N} \sum a_{j}{ }^{2}\right\} \\
\times\left[1+\sum_{k=1}^{6} \alpha_{k-1}\left(\frac{\lambda(1-\lambda) \sum a_{j}{ }^{2}}{N}\right)^{\frac{1}{2} k}(i t)^{k}\right] .
\end{gathered}
$$

Because $\max \left|a_{j}\right| \leqq(C N)^{\frac{1}{4}}$ we find by the same reasoning as in (3.22),

$$
\begin{aligned}
N^{-1} E_{H} \mid U \sum & a_{j}{ }^{2}\left(P_{j}-\pi_{j}\right) \mid+N^{-2} E_{H}\left\{\sum a_{j}{ }^{2}\left(P_{j}-\pi_{j}\right)\right\}^{2} \\
& \leqq B_{5} N^{-\frac{5}{4}} E_{H}\left\{\sum\left|a_{j}\left(P_{j}-\pi_{j}\right)\right|\right\}^{2} \\
& \leqq B_{5} N^{-\frac{5}{4}}\left[1+E_{H}\left\{\sum\left|a_{j}\left(P_{j}-\pi_{j}\right)\right|\right\}^{3}\right] \\
& \leqq B_{5} N^{-\frac{5}{4}}+B_{8} N^{-\frac{1}{2}}\left[\sum\left\{E_{H}\left|P_{j}-\pi_{j}\right|^{3}\right\}^{\frac{4}{5}}\right]^{\frac{9}{4}} .
\end{aligned}
$$

Together with (3.22) this shows that (3.23) may be reduced to

$$
\begin{gathered}
\left|\rho^{*}(t)-\bar{\rho}(t)\right| \leqq B_{7}|t| \exp \left\{-\beta_{3} t^{2}\right\}\left\{N^{-\frac{5}{4}}+N^{\frac{1}{2}} E_{H}\left(\frac{g\left(X_{1}\right)}{h\left(X_{1}\right)}-1\right)^{4}\right. \\
\left.+N^{-\frac{1}{2}}\left[\sum\left\{E_{H}\left|P_{j}-\pi_{j}\right|^{3}\right\}^{\frac{4}{4}}\right]^{\frac{9}{4}}\right\}
\end{gathered}
$$

As $\alpha_{0}, \cdots, \alpha_{5}$ are bounded and $N^{-\frac{1}{2}}\left|\sum^{\prime} a_{j} \pi_{j}\right| \leqq C^{\frac{1}{4}} N^{\frac{1}{2}}$, we have $\left|\bar{\rho}^{\prime}(t)\right| \leqq B_{8} N^{\frac{1}{2}}$ for all $t$. Since $\left|\rho^{\prime}(t)\right| \leqq N^{-\frac{1}{2}} E|T| \leqq C^{\frac{1}{4}} N^{\frac{1}{2}}$ for all $t$ and $\rho(0)=\bar{\rho}(0)=1$,

$$
|\rho(t)-\bar{\rho}(t)| \leqq B_{9} N^{\frac{1}{2}}|t| \quad \text { for all } t \text {. }
$$

Combining Lemma 3.3, (3.25) and (3.26) we find

$$
\begin{aligned}
\int_{-b N^{\frac{3}{2}}}^{b N^{\frac{3}{2}}} \mid & \left|\frac{\rho(t)-\bar{\rho}(t)}{t}\right| d t \\
& \leqq B_{9} N^{-\frac{3}{2}}+\int_{N^{-2} \leqq|t| \leqq b N^{\frac{3}{2}}}\left|\frac{\rho(t)-\bar{\rho}(t)}{t}\right| d t \\
& \leqq B_{10}\left\{N^{-\frac{5}{4}}+N^{\frac{3}{4}} E_{H}\left(\frac{g\left(X_{1}\right)}{h\left(X_{1}\right)}-1\right)^{4}+N^{-\frac{1}{2}}\left[\sum\left\{E_{H}\left|P_{j}-\pi_{j}\right|^{3}\right\}^{\frac{3}{5}}\right]^{\frac{9}{2}}\right\} .
\end{aligned}
$$


Now $\bar{\rho}(t)$ is the Fourier-Stieltjes transform of $K\left(\left\{N^{\frac{1}{2}} x-\sum a_{j} \pi_{j}\right\}\left\{\lambda(1-\lambda) \sum a_{j}\right\}^{-\frac{1}{2}}\right)$ as a function of $x$. This is a function of bounded variation assuming the values 0 and 1 at $-\infty$ and $+\infty$ and having a derivative that is bounded by $3 B_{3} c^{-\frac{1}{2}}\{\varepsilon(1-\varepsilon)\}^{-1}$ in absolute value. It follows from the smoothing lemma (Esseen (1945)) that

$$
\sup _{x}\left|P\left(N^{-\frac{1}{2}} T \leqq x\right)-K\left(\frac{N^{\frac{1}{2}} x-\sum a_{j} \pi_{j}}{\left\{\lambda(1-\lambda) \sum a_{j}{ }^{2}\right\}^{\frac{1}{2}}}\right)\right|
$$

is bounded above by the right-hand side of (3.20). A change of scale completes the proof.

Theorem 3.1 provides the basic expansion for the distribution of $T$ under contiguous alternatives. Only first and second moments of functions of order statistics remain to be determined. In Section 4 we shall be concerned with a further simplification of the expansion and a precise evaluation of the order of the remainder. With regard to this remainder we are in a seemingly less favorable position than we were at the same stage in the one-sample problem (cf. ABZ (1976), Theorem 2.3), because the third remainder term in (3.20) is larger than the corresponding term in the one-sample case by a factor $N^{\frac{1}{4}}$. This is due to the appearance of the remainder term $N^{-1} E_{H}\left|U \sum a_{j}{ }^{2}\left(P_{j}-\pi_{j}\right)\right|$ that does not occur for the one-sample statistic. It will turn out, however, that we shall need only a slightly stronger condition than before to show that the remainder is still $O\left(N^{-\frac{5}{4}}\right)$.

The conditions of Theorem 3.1 concern only the sample ratio $\lambda$ and the scores $a$. There are no assumptions about the underlying densities $f$ and $g$ but this is merely a trick; obviously something like contiguity is needed to make the expansion meaningful in the sense that the remainder is at all small. With regard to the conditions on the scores, (3.10) acts as a safeguard against too rapid growth and (2.62) ensures that the $a_{j}$ do not cluster too much around too few points, thus preventing a too pronounced lattice character of the distribution of $T$, as was pointed out in $\mathrm{ABZ}$ (1976). It was also noted there that in the important case of exact scores $a_{j}=E J\left(U_{j: N}\right)$, with $U_{1: N}<U_{2: N}<\cdots<U_{N: N}$ order statistics from the uniform distribution on $(0,1)$, both $(3.10)$ and $(2.62)$ will be satisfied for all $N$ with fixed $c, C$ and $\delta$ if $J$ is a continuously differentiable, nonconstant function on $(0,1)$ with $\int J^{4}<\infty$. The same is true for approximate scores $a_{j}=J(j /(N+1))$ provided that $J$ is monotone near 0 and 1 .

4. Contiguous location alternatives. The analysis in this section will be carried out for contiguous location alternatives rather than for contiguous alternatives in general. The general case can be treated in much the same way as the location case, but the conditions as well as the results become more involved.

We recall some assumptions and notation from Section 3 of ABZ (1976). Let $F$ be a df with a density $f$ that is positive on $R^{1}$ and four times differentiable with derivatives $f^{(i)}, i=1, \ldots, 4$. Define

$$
\phi_{i}=\frac{f^{(i)}}{f}, \quad i=1, \ldots, 4,
$$


and suppose that positive numbers $\varepsilon^{\prime}$ and $C^{\prime}$ exist such that for

$$
\begin{aligned}
& m_{1}=6, \quad m_{2}=3, \quad m_{3}=\frac{4}{3}, \quad m_{4}=1, \\
& \sup \left\{\int_{-\infty}^{\infty}\left|\psi_{i}(x+y)\right|^{m_{\imath}} f(x) d x:|y| \leqq \varepsilon^{\prime}\right\} \leqq C^{\prime}, \quad i=1, \cdots, 4 .
\end{aligned}
$$

So far, we have studied the distribution of $T$ under the assumption that $X_{1}, \cdots, X_{N}$ are independent, $X_{1}, \cdots, X_{m}$ having common $\mathrm{df} F$ and $X_{m+1}, \cdots, X_{N}$ having $\mathrm{df} G$. We now add the assumptions that

$$
G(x)=F(x-\theta)
$$

for all $x$ and that

$$
0 \leqq \theta \leqq D N^{-\frac{1}{2}}
$$

for some $D>0$. Probabilities under this particular model will still be denoted by $P$. Note that (4.2), (4.3) and (4.4) together imply contiguity.

In Section 3 we also introduced an auxiliary model where $X_{1}, \ldots, X_{N}$ are supposed to be i.i.d. with common df $H=(1-\lambda) F+\lambda G$. In view of (4.3) this common df now becomes $H(x)=(1-\lambda) F(x)+\lambda F(x-\theta)$. Probabilities, expectations and variances under this model will be denoted by $P_{H}, E_{H}$ and $\sigma_{H}{ }^{2}$ as before. Similarly, $P_{F}, E_{F}$ and $\sigma_{F}{ }^{2}$ will indicate probabilities, expectations and variances under a third model where $X_{1}, \cdots, X_{N}$ are i.i.d. with common df $F$. Note that for $\theta=0$ these three models coincide.

Define

$$
\tilde{K}(x)=\Phi(x)-\phi(x) \sum_{k=0}^{5} \tilde{\alpha}_{k} H_{k}(x)
$$

where

$$
\begin{aligned}
& \tilde{\alpha}_{0}=\frac{1}{6}\left(\frac{\lambda(1-\lambda)}{\sum a_{j}{ }^{2}}\right)^{\frac{1}{2}}\left[3(1-2 \lambda) \theta^{2} \sum a_{j} E_{F} \psi_{2}\left(Z_{j}\right)-6 N^{-1} \theta \sum a_{j} E_{F} \psi_{1}\left(Z_{j}\right)\right. \\
& -\theta^{3} \sum a_{j} E_{F}\left\{\left(1-3 \lambda+3 \lambda^{2}\right) \psi_{3}\left(Z_{j}\right)-6 \lambda(1-\lambda) \psi_{1}\left(Z_{j}\right) \psi_{2}\left(Z_{j}\right)\right. \\
& \left.\left.+3 \lambda(1-\lambda) \psi_{1}^{3}\left(Z_{j}\right)\right\}\right] \text {, } \\
& \tilde{\alpha}_{1}=\frac{1}{8 \sum a_{j}{ }^{2}}\left[-4(1-2 \lambda) \theta \sum a_{j}{ }^{2} E_{F} \psi_{1}\left(Z_{j}\right)+2(1-2 \lambda)^{2} \theta^{2} \sum a_{j}{ }^{2} E_{F} \psi_{2}\left(Z_{j}\right)\right. \\
& -4 \lambda(1-\lambda) \theta^{2} \sum a_{j}^{2} E_{F} \psi_{1}^{2}\left(Z_{j}\right)+4 \lambda(1-\lambda) \theta^{2} \sigma_{F}^{2}\left(\sum a_{j} \psi_{1}\left(Z_{j}\right)\right) \\
& -4(1-2 \lambda)^{2} N^{-1} \theta^{2}\left\{\sum a_{j} E_{F} \psi_{1}\left(Z_{j}\right)\right\}^{2} \\
& \left.+\lambda(1-\lambda)(1-2 \lambda)^{2} \theta^{4}\left\{\sum a_{j} E_{F} \psi_{2}\left(Z_{j}\right)\right\}^{2}\right]+\frac{1}{2 N}, \\
& \tilde{\alpha}_{2}=\frac{1}{12\{\lambda(1-\lambda)\}^{\frac{1}{2}}\left(\sum a_{j}{ }^{2}\right)^{\frac{3}{2}}}\left[2(1-2 \lambda) \sum a_{j}{ }^{3}-2\left(1-6 \lambda+6 \lambda^{2}\right)\right. \\
& \times \theta \sum a_{j}{ }^{3} E_{F} \psi_{1}\left(Z_{j}\right)+6(1-2 \lambda)^{2} N^{-1} \theta \sum a_{j}{ }^{2} \sum a_{j} E_{F} \psi_{1}\left(Z_{j}\right) \\
& \left.-3 \lambda(1-\lambda)(1-2 \lambda)^{2} \theta^{3} \sum a_{j}^{2} E_{F} \psi_{1}\left(Z_{j}\right) \sum a_{j} E_{F} \psi_{2}\left(Z_{j}\right)\right] \text {, } \\
& \tilde{\alpha}_{3}=\frac{1}{24 \lambda(1-\lambda)\left(\sum a_{j}{ }^{2}\right)^{2}}\left\{\left(1-6 \lambda+6 \lambda^{2}\right) \sum a_{j}{ }^{4}+3 \lambda(1-\lambda)(1-2 \lambda)^{2} \theta^{2}\right. \\
& \left.\times\left\{\sum a_{j}{ }^{2} E_{F} \psi_{1}\left(Z_{j}\right)\right\}^{2}+2 \lambda(1-\lambda)(1-2 \lambda)^{2} \theta^{2} \sum a_{j}{ }^{3} \sum a_{j} E_{F} \psi_{2}\left(Z_{j}\right)\right] \\
& -\frac{(1-2 \lambda)^{2}}{8 \lambda(1-\lambda) N}
\end{aligned}
$$




$$
\begin{aligned}
& \tilde{\alpha}_{4}=-\frac{(1-2 \lambda)^{2} \theta \sum a_{j}{ }^{3} \sum a_{j}{ }^{2} E_{F} \psi_{1}\left(Z_{j}\right)}{12\{\lambda(1-\lambda)\}^{\frac{1}{2}}\left(\sum a_{j}{ }^{2}\right)^{\frac{5}{2}}}, \\
& \tilde{\alpha}_{5}=\frac{(1-2 \lambda)^{2}\left(\sum a_{j}{ }^{3}\right)^{2}}{72 \lambda(1-\lambda)\left(\sum a_{j}{ }^{3}\right)^{3}},
\end{aligned}
$$

and let

$$
\eta=-\left(\frac{\lambda(1-\lambda)}{\sum a_{j}^{2}}\right)^{\frac{1}{2}} \theta \sum a_{j} E_{F} \psi_{1}\left(Z_{j}\right)
$$

We shall show that $\tilde{K}(x-\eta)$ is an expansion for the $\mathrm{df}$ of $\left\{\lambda(1-\lambda) \sum a_{j}{ }^{2}\right\}^{-\frac{1}{2}} T$. The expansion will be established in Theorem 4.1 and an evaluation of the order of the remainder will be given in Theorem 4.2.

Let $\pi(F, \theta)$ denote the power of the one-sided level $\alpha$ test based on $T$ for the hypothesis $F=G$ against the alternative $G(x)=F(x-\theta)$. Suppose that

$$
\varepsilon^{\prime \prime} \leqq \alpha \leqq 1-\varepsilon^{\prime \prime},
$$

for some $\varepsilon^{\prime \prime}>0$. We shall prove that an expansion for $\pi(F, \theta)$ is given by

$$
\tilde{\pi}(F, \theta)=1-\Phi\left(u_{\alpha}-\eta\right)+\phi\left(u_{\alpha}-\eta\right) \sum_{k=0}^{5} \tilde{\beta}_{k} H_{k}\left(u_{\alpha}-\eta\right),
$$

where $u_{\alpha}=\Phi^{-1}(1-\alpha)$ is the upper $\alpha$-point of the standard normal distribution and

$$
\begin{aligned}
& \tilde{\beta}_{0}= \tilde{\alpha}_{0}-\frac{(1-2 \lambda) \sum a_{j}{ }^{3}}{6\{\lambda(1-\lambda)\}^{\frac{1}{2}}\left(\sum a_{j}{ }^{2}\right)^{\frac{3}{2}}}\left(u_{\alpha}{ }^{2}-1\right)+2 \tilde{\alpha}_{5}\left(2 u_{\alpha}{ }^{3}-5 u_{\alpha}\right)-\frac{u_{\alpha}}{2 N} \\
&-\left\{\frac{\left(1-6 \lambda+6 \lambda^{2}\right) \sum a_{j}^{4}}{24 \lambda(1-\lambda)\left(\sum a_{j}^{2}\right)^{2}}-\frac{(1-2 \lambda)^{2}}{8 \lambda(1-\lambda) N}\right\}\left(u_{\alpha}{ }^{3}-3 u_{\alpha}\right), \\
& \tilde{\beta}_{1}= \tilde{\alpha}_{1}+\tilde{\alpha}_{5}\left(u_{\alpha}{ }^{2}-1\right)^{2}-\frac{(1-2 \lambda)^{2}}{12\left(\sum a_{j}{ }^{2}{ }^{2}\right.} \theta^{2} \sum a_{j}{ }^{3} \sum a_{j} E_{F} \psi_{2}\left(Z_{j}\right)\left(u_{\alpha}{ }^{2}-1\right), \\
& \tilde{\beta}_{2}=\tilde{\alpha}_{2}-\tilde{\alpha}_{4}\left(u_{\alpha}{ }^{2}-1\right), \\
& \tilde{\beta}_{3}=\tilde{\alpha}_{3}-2 \tilde{\alpha}_{5}\left(u_{\alpha}{ }^{2}-1\right), \\
& \tilde{\beta}_{k}=\tilde{\alpha}_{k} \quad \text { for } \quad k=4,5 .
\end{aligned}
$$

Theorem 4.1. Suppose that (3.1) and (4.3) hold and that positive numbers $c, C$, $C^{\prime}, D, \delta, \varepsilon$ and $\varepsilon^{\prime}$ exist such that (3.10), (2.62), (3.19), (4.2) and (4.4) are satisfied. Define

$$
\begin{aligned}
M= & N^{-\frac{5}{4}}+N^{-\frac{1}{2}} \theta^{3}\left[\sum\left\{E_{F}\left|\psi_{1}\left(Z_{j}\right)-E_{F} \psi_{1}\left(Z_{j}\right)\right|^{3}\right\}^{\frac{4}{3}}\right]^{\frac{9}{4}} \\
& +N^{-\frac{3}{4}} \theta^{3}\left[\sum\left\{E_{F}\left(\psi_{2}\left(Z_{j}\right)-E_{F} \psi_{2}\left(Z_{j}\right)\right)^{2}\right\}^{\frac{2}{3}}\right]^{\frac{3}{2}} .
\end{aligned}
$$

Then there exists $B>0$ depending only on $c, C, C^{\prime}, D, \delta, \varepsilon$ and $\varepsilon^{\prime}$ such that

$$
\sup _{x}\left|P\left(\frac{T}{\left\{\lambda(1-\lambda) \sum a_{j}^{2}\right\}^{\frac{1}{2}}} \leqq x\right)-\tilde{K}(x-\eta)\right| \leqq B M
$$

If, in addition, (4.8) is satisfied there exists $B^{\prime}>0$ depending only on $c, C, C^{\prime}, D$, $\delta, \varepsilon, \varepsilon^{\prime}$ and $\varepsilon^{\prime \prime}$ such that

$$
|\pi(F, \theta)-\tilde{\pi}(F, \theta)| \leqq B^{\prime} M
$$


Proof. The proof of (4.12) hinges on Taylor expansion with respect to $\theta$ of the moments under $P_{H}$ of functions of $P=\left(P_{1}, \cdots, P_{N}\right)$ occurring in expansion (3.20). Since both $H$ and $P$ depend on $\theta$ the argument is highly technical and laborious and it is therefore given in the appendix. Theorem 3.1, Corollary A.1, (A.12) and (A.13) immediately yield (4.12).

The one-sided level $\alpha$ test based on $T$ rejects the hypothesis if $T\left\{\lambda(1-\lambda) \sum a_{j}^{2}\right\}^{-\frac{1}{2}} \geqq \xi_{\alpha}$ with possible randomization if equality occurs. Using (4.12) for $\theta=0$ (or Corollary 2.1), (3.10), (3.19) and (4.8) we easily show that

$$
\begin{aligned}
\xi_{\alpha}=u_{\alpha} & +\frac{(1-2 \lambda) \sum a_{j}{ }^{3}}{6\{\lambda(1-\lambda)\}^{\frac{1}{2}}\left(\sum a_{j}{ }^{2}\right)^{\frac{3}{2}}}\left(u_{\alpha}{ }^{2}-1\right)-2 \tilde{\alpha}_{5}\left(2 u_{\alpha}{ }^{3}-5 u_{\alpha}\right)+\frac{u_{\alpha}}{2 N} \\
& +\left\{\frac{\left(1-6 \lambda+6 \lambda^{2}\right) \sum a_{j}{ }^{4}}{24 \lambda(1-\lambda)\left(\sum a_{j}{ }^{2}\right.}-\frac{(1-2 \lambda)^{2}}{8 \lambda(1-\lambda) N}\right\}\left(u_{\alpha}{ }^{3}-3 u_{\alpha}\right)+O\left(N^{-\frac{5}{4}}\right),
\end{aligned}
$$

where, in this proof, $O(x)$ denotes a quantity bounded by $B_{1}|x|$ with $B_{1}$ depending only on $c, C, C^{\prime}, D, \delta, \varepsilon, \varepsilon^{\prime}$ and $\varepsilon^{\prime \prime}$. Because of (4.12),

$$
\pi(F, \theta)=1-\tilde{K}\left(\xi_{\alpha}-\eta\right)+O(M) .
$$

Using (4.14), (4.8) and the bounds provided by Corollary A.1, we now expand $\tilde{K}\left(\xi_{\alpha}-\eta\right)$ about the point $\left(u_{\alpha}-\eta\right)$ and arrive at (4.13).

Define

$$
\Psi_{i}(t)=\phi_{i}\left(F^{-1}(t)\right)=\frac{f^{(2)}\left(F^{-1}(t)\right)}{f\left(F^{-1}(t)\right)}, \quad i=1, \ldots, 4 .
$$

THEOREM 4.2. Let $M$ be defined by (4.11) and suppose that positive numbers $D$, $C$ and $\delta$ exist such that (4.4) is satisfied and that $\left|\Psi_{1}^{\prime}(t)\right| \leqq C\{t(1-t)\}^{-\frac{5}{4}+\delta}$ and $\left|\Psi_{2}{ }^{\prime}(t)\right| \leqq C\{t(1-t)\}^{-\frac{3}{2}+\delta}$. Then there exist $B>0$ depending only $D, C$ and $\delta$ such that

$$
M \leqq B N^{-\frac{5}{3}} .
$$

Proof. The proof is similar to that of Corollary A2.1 in ABZ (1976). To deal with the second term of $M$ we take $h=\Psi_{1}$ and replace $\frac{4}{3}$ by $\frac{5}{4}$ in the proof of that corollary. For the third term of $M$ we take $h=\Psi_{2}$, replace $\frac{4}{3}$ by $\frac{3}{2}$, appeal to condition $R_{2}$ instead of $R_{3}$ and otherwise proceed as in the proof of Corollary A2.1 of ABZ (1976).

5. Exact and approximate scores. A further simplification of the expansions in Section 4 may obtained if we make certain smoothness assumptions about the scores $a_{j}$. Consider a continuous function $J$ on $(0,1)$ and let $U_{1: N}<U_{2: N}<\ldots$ $<U_{N: N}$ denote order statistics of a sample of size $N$ from the uniform distribution on $(0,1)$. For $N=1,2, \ldots$ we define the exact scores generated by $J$ by

$$
a_{j}=a_{j, N}=E J\left(U_{j: N}\right),
$$$$
j=1, \cdots, N \text {, }
$$

and the approximate scores generated by $J$ by

$$
a_{j}=a_{j, N}=J\left(\frac{j}{N+1}\right), \quad j=1, \cdots, N .
$$


For exact scores and general $J$ Theorem 5.1 will provide expansions for the $\mathrm{df}$ of $T$ under contiguous location alternatives of type $F$ and for the power of the rank test against these alternatives. In Theorem 5.2 we consider the special case $J=-\Psi_{1}$, with $\Psi_{1}$ as in (4.15), for exact as well as approximate scores. Note that the exact scores generated by $-\Psi_{1}$ define the locally most powerful rank test.

As in Section 4 of ABZ (1976) it is now no longer feasible to keep the order of the remainder in our expansions down to $O\left(N^{-\frac{5}{4}}\right)$ and we shall be content with $o\left(N^{-1}\right)$. Also as in ABZ (1976) we shall formulate the results in this section for a fixed scores generating function $J$ and a fixed df $F$, leaving the construction of uniformity classes to the reader.

Definition 5.1. $\mathscr{J}$ is the class of functions $J$ on $(0,1)$ that are twice continuously differentiable and nonconstant on $(0,1)$ and satisfy

$$
\begin{gathered}
\int_{0}^{1} J(t) d t=0, \\
\lim _{t \rightarrow 0,1}\{t(1-t)\}^{\frac{7}{5}} J^{\prime}(t)=0, \\
\lim \sup _{t \rightarrow 0,1} t(1-t)\left|\frac{J^{\prime \prime}(t)}{J^{\prime}(t)}\right|<\frac{3}{2} .
\end{gathered}
$$

$\mathscr{F}$ is the class of df's $F$ on $R^{1}$ with positive and four times differentiable densities $f$ and such that, for $\phi_{i}=f^{(i)} / f, \Psi_{i}=\phi_{i}\left(F^{-1}\right), m_{1}=6, m_{2}=3, m_{3}=\frac{4}{3}$, $m_{4}=1$,

$$
\begin{gathered}
\lim \sup _{y \rightarrow 0} \int_{-\infty}^{\infty}\left|\psi_{i}(x+y)\right|^{m} i f(x) d x<\infty, \quad i=1, \cdots, 4, \\
\lim \sup _{t \rightarrow 0,1} t(1-t)\left|\frac{\Psi_{1}^{\prime \prime}(t)}{\Psi^{\prime}(t)}\right|<\frac{3}{2} .
\end{gathered}
$$

Note that one can argue as in the proof of Corollary A2.1 of ABZ (1976) to show that, in conjunction with (5.5), condition (5.4) is weaker than the assumption $\int J^{6}(t) d t<\infty$. Define

$$
\begin{aligned}
& \bar{\alpha}_{0}=\frac{1}{6}\left(\frac{\lambda(1-\lambda)}{N \int J^{2}(t) d t}\right)^{\frac{1}{2}}\left[3(1-2 \lambda) N \theta^{2} \int J(t) \Psi_{2}(t) d t-6 \theta \int J(t) \Psi_{1}(t) d t\right. \\
&-N \theta^{3} \int J(t)\left\{\left(1-3 \lambda+3 \lambda^{2}\right) \Psi_{3}(t)-6 \lambda(1-\lambda) \Psi_{1}(t) \Psi_{2}(t)\right. \\
&\left.\left.+3 \lambda(1-\lambda) \Psi_{1}^{3}(t)\right\} d t\right] \\
& \bar{\alpha}_{1}=\frac{1}{8 \int J^{2}(t) d t}\left[-4(1-2 \lambda) \theta \int J^{2}(t) \Psi_{1}(t) d t\right. \\
& \quad+2(1-2 \lambda)^{2} \theta^{2} \int J^{2}(t) \Psi_{2}(t) d t-4 \lambda(1-\lambda) \theta^{2} \int J^{2}(t) \Psi_{1}^{2}(t) d t \\
& \quad+4 \lambda(1-\lambda) \theta^{2} \iint J(s) J(t) \Psi_{1}^{\prime}(s) \Psi_{1}^{\prime}(t)[s \wedge t-s t] d s d t \\
& \quad-4(1-2 \lambda)^{2} \theta^{2}\left\{\int J(t) \Psi_{1}(t) d t\right\}^{2} \\
&\left.\quad+\lambda(1-\lambda)(1-2 \lambda)^{2} N \theta^{4}\left\{\int J(t) \Psi_{2}(t) d t\right\}^{2}\right]+\frac{1}{2 N},
\end{aligned}
$$




$$
\begin{aligned}
& \bar{\alpha}_{2}=\frac{1}{12\{\lambda(1-\lambda) N\}^{\frac{1}{2}}\left\{\int J^{2}(t) d t\right\}^{\frac{3}{2}}}\left[2(1-2 \lambda) \int J^{3}(t) d t\right. \\
& -2\left(1-6 \lambda+6 \lambda^{2}\right) \theta \int J^{3}(t) \Psi_{1}(t) d t \\
& +6(1-2 \lambda)^{2} \theta \int J^{2}(t) d t \int J(t) \Psi_{1}(t) d t \\
& \left.-3 \lambda(1-\lambda)(1-2 \lambda)^{2} N \theta^{3} \int J^{2}(t) \Psi_{1}(t) d t \int J(t) \Psi_{2}(t) d t\right], \\
& \bar{\alpha}_{3}=\frac{1}{24 \lambda(1-\lambda) N\left\{\int J^{2}(t) d t\right\}^{2}}\left[\left(1-6 \lambda+6 \lambda^{2}\right) \int J^{4}(t) d t\right. \\
& +3 \lambda(1-\lambda)(1-2 \lambda)^{2} N \theta^{2}\left\{\int J^{2}(t) \Psi_{1}(t) d t\right\}^{2} \\
& \left.+2 \lambda(1-\lambda)(1-2 \lambda)^{2} N \theta^{2} \int J^{3}(t) d t \int J(t) \Psi_{2}(t) d t\right]-\frac{(1-2 \lambda)^{2}}{8 \lambda(1-\lambda) N}, \\
& \bar{\alpha}_{4}=-\frac{(1-2 \lambda)^{2} \theta}{12\{\lambda(1-\lambda) N\}^{\frac{1}{2}}} \frac{\int J^{3}(t) d t \int J^{2}(t) \Psi_{1}(t) d t}{\left\{\int J^{2}(t) d t\right\}^{\frac{3}{2}}}, \\
& \bar{\alpha}_{5}=\frac{(1-2 \lambda)^{2}}{72 \lambda(1-\lambda) N} \frac{\left\{\int J^{3}(t) d t\right\}^{2}}{\left\{\int J^{2}(t) d t\right\}^{3}}, \\
& \bar{K}_{1}(x)=\Phi(x)-\phi(x)\left\lceil\sum_{k=0}^{5} \bar{\alpha}_{k} H_{k}(x)\right. \\
& +\frac{1}{2}\left(\frac{\lambda(1-\lambda)}{N \int J^{2}(t) d t}\right)^{\frac{1}{2}} \theta\left\{2 \sum_{j=1}^{N} \operatorname{Cov}\left(J\left(U_{j: N}\right), \Psi_{1}\left(U_{j: N}\right)\right)\right. \\
& \left.\left.-\frac{\int J(t) \Psi_{1}(t) d t}{\int J^{2}(t) d t} \sum_{j=1}^{N} \sigma^{2}\left(J\left(U_{j: N}\right)\right)\right\}\right] \\
& \bar{K}_{2}(x)=\Phi(x)-\phi(x)\left[\sum_{k=0}^{5} \bar{\alpha}_{k} H_{k}(x)\right. \\
& +\frac{1}{2}\left(\frac{\lambda(1-\lambda)}{N \int J^{2}(t) d t}\right)^{\frac{1}{2}} \theta\left\{2 \int_{N^{-1}}^{1-N-1} J^{\prime}(t) \Psi_{1}^{\prime}(t) t(1-t) d t\right. \\
& \left.\left.-\frac{\int J(t) \Psi_{1}(t) d t}{\int J^{2}(t) d t} \int_{N-1}^{1-N-1}\left(J^{\prime}(t)\right)^{2} t(1-t) d t\right\}\right] \\
& \bar{\eta}=-\left(\frac{\lambda(1-\lambda) N}{\int J^{2}(t) d t}\right)^{\frac{1}{2}} \theta \int J(t) \Psi_{1}(t) d t,
\end{aligned}
$$
where all integrals are over $(0,1)$ unless otherwise indicated. We shall show
that $\bar{K}_{1}(x-\bar{\eta})$ and $\bar{K}_{2}(x-\bar{\eta})$ are expansions for the df of $\left\{\lambda(1-\lambda) \sum a_{j}{ }^{2}\right\}^{-\frac{1}{2}} T$ for exact scores. Furthermore let

$$
\begin{aligned}
\bar{\beta}_{0}=\bar{\alpha}_{0} & -\frac{(1-2 \lambda)}{6\{\lambda(1-\lambda) N\}^{\frac{1}{2}}} \frac{\int J^{3}(t) d t}{\left\{\int J^{2}(t) d t\right\}^{\frac{3}{2}}}\left(u_{\alpha}{ }^{2}-1\right)+2 \bar{\alpha}_{5}\left(2 u_{\alpha}{ }^{3}-5 u_{\alpha}\right)-\frac{u_{\alpha}}{2 N} \\
& -\left\{\frac{\left(1-6 \lambda+6 \lambda^{2}\right)}{24 \lambda(1-\lambda) N} \frac{\int J^{4}(t) d t}{\left\{\int J^{2}(t) d t\right\}^{2}}-\frac{(1-2 \lambda)^{2}}{8 \lambda(1-\lambda) N}\right\}\left(u_{\alpha}{ }^{3}-3 u_{\alpha}\right),
\end{aligned}
$$

$$
\bar{\beta}_{1}=\bar{\alpha}_{1}+\bar{\alpha}_{5}\left(u_{\alpha}{ }^{2}-1\right)^{2}-\frac{(1-2 \lambda)^{2} \theta^{2}}{12} \frac{\int J^{3}(t) d t \int J(t) \Psi_{2}(t) d t}{\left\{\int J^{2}(t) d t\right\}^{2}}\left(u_{\alpha}{ }^{2}-1\right),
$$




$$
\begin{aligned}
& \bar{\beta}_{2}=\bar{\alpha}_{2}-\bar{\alpha}_{4}\left(u_{\alpha}{ }^{2}-1\right), \\
& \bar{\beta}_{3}=\bar{\alpha}_{3}-2 \bar{\alpha}_{5}\left(u_{\alpha}{ }^{2}-1\right), \\
& \bar{\beta}_{k}=\bar{\alpha}_{k} \quad \text { for } \quad k=4,5, \\
& \begin{aligned}
\bar{\pi}_{i}(F, \theta)=1 & -\bar{K}_{i}\left(u_{\alpha}-\bar{\eta}\right) \\
& \quad+\phi\left(u_{\alpha}-\bar{\eta}\right) \sum_{k=0}^{5}\left(\tilde{\beta}_{k}-\bar{\alpha}_{k}\right) H_{k}\left(u_{\alpha}-\bar{\eta}\right), \quad i=1,2,
\end{aligned}
\end{aligned}
$$

i.e., $\bar{\pi}_{i}(F, \theta)$ equals $1-\bar{K}_{i}\left(u_{\alpha}-\tilde{\eta}\right)$ with $\bar{\alpha}_{k}$ replaced by $\bar{\beta}_{k}, k=0, \ldots, 3$.

Theorem 5.1. Let $F \in \mathscr{F}, J \in \mathscr{F}, a_{j}=E J\left(U_{j: N}\right)$ for $j=1, \ldots, N, G(x)=$ $F(x-\theta), 0 \leqq \theta \leqq D N^{-\frac{1}{2}}, \varepsilon \leqq \lambda \leqq 1-\varepsilon$ and $\varepsilon^{\prime} \leqq \alpha \leqq 1-\varepsilon^{\prime}$ for positive $D, \varepsilon$ and $\varepsilon^{\prime}$. Then, for every fixed $F, J, D, \varepsilon$ and $\varepsilon^{\prime}$, there exist positive numbers $B$, $\delta_{1}, \delta_{2}, \ldots$ such that $\lim _{N \rightarrow \infty} \delta_{N}=0$ and for every $N$

$$
\begin{aligned}
& \sup _{x}\left|P\left(\frac{T}{\left\{\lambda(1-\lambda) \sum a_{j}^{2}\right\}^{\frac{1}{2}}} \leqq x\right)-\bar{K}_{1}(x-\bar{\eta})\right| \leqq \delta_{N} N^{-1}, \\
& \sup _{x}\left|P\left(\frac{T}{\left\{\lambda(1-\lambda) \sum a_{j}^{2}\right\}^{\frac{1}{2}}} \leqq x\right)-\bar{K}_{2}(x-\bar{\eta})\right| \\
& \quad \leqq \delta_{N} N^{-1}+B N^{-\frac{3}{2}} \int_{N^{-1}}^{1-1}\left|J^{\prime}(t)\right|\left(\left|J^{\prime}(t)\right|+\left|\Psi_{1}^{\prime}(t)\right|\right)\{t(1-t)\}^{\frac{1}{2}} d t, \\
& \quad\left|\pi(F, \theta)-\bar{\pi}_{1}(F, \theta)\right| \leqq \delta_{N} N^{-1}, \\
& \quad \leqq \pi(F, \theta)-\bar{\pi}_{2}(F, \theta) \mid \\
& \quad \delta_{N} N^{-1}+B N^{-\frac{3}{2}} \int_{N^{-1}}^{1-N^{-1}}\left|J^{\prime}(t)\right|\left(\left|J^{\prime}(t)\right|+\left|\Psi_{1}^{\prime}(t)\right|\right)\{t(1-t)\}^{\frac{1}{2}} d t .
\end{aligned}
$$

Proof. In the first part of the proof we shall not need requirement (5.4) but only the weaker assumption $\int J^{4}(t) d t<\infty$. We proceed as in the proof of Theorem 4.1 in ABZ (1976), drawing heavily on the results in Appendix 2 of ABZ (1976). Note that these results remain valid in the present context even though the definition of the functions $\Psi_{i}$ is slightly different here. Throughout the proof we shall make use of $O$ and $o$ symbols that are uniform for fixed $F$, $J, D, \varepsilon$ and $\varepsilon^{\prime}$.

Because $\sum a_{j}=N \int J(t) d t=0$ and in view of the remark made at the end of Section 3, the assumptions of Theorem 4.1 are satisfied. The proof of Corollary A2.1 of ABZ (1976) shows that (5.6) and (5.7) imply that

$$
\Psi_{1}{ }^{\prime}(t)=o\left(\{t(1-t)\}^{-\frac{7}{6}}\right) \quad \text { for } \quad t \rightarrow 0,1 .
$$

Hence, because of $(5.7), \Psi_{1}^{\prime \prime}(t)=o\left(\{t(1-t)\}^{-\frac{18}{6}}\right)$ and $\Psi_{1}(t)=o\left(\{t(1-t)\}^{-\frac{1}{6}}\right)$ for $t \rightarrow 0,1$. Since $f\left(F^{-1}\right)$ has a summable derivative $\Psi_{1}$ on $(0,1), f\left(F^{-1}\right)$ must have limits at 0 and 1 ; as $f$ is positive on $R^{1}$, these limits must be equal to 0 . It follows that $f\left(F^{-1}(t)\right)=o\left(\{t(1-t)\}^{8}\right)$ for $t \rightarrow 0,1$. Combining these facts with the identity $\Psi_{2}^{\prime}(t)=\Psi_{1}^{\prime \prime}(t) f\left(F^{-1}(t)\right)+3 \Psi_{1}(t) \Psi_{1}^{\prime}(t)$, we find that

$$
\Psi_{2}{ }^{\prime}(t)=o\left(\{t(1-t)\}^{-4}\right) \quad \text { for } \quad t \rightarrow 0,1 .
$$

Thus the assumptions of Theorem 4.2 are also satisfied and we can take the expansions of Section 4 as a starting point for proving Theorem 5.1. 
In $\tilde{\alpha}_{0}, \cdots, \tilde{\alpha}_{5}, \tilde{\beta}_{0}, \cdots, \tilde{\beta}_{5}$ defined by (4.6) and (4.10) we may replace $E_{F}, \sigma_{F}^{3}$ and $\phi_{i}\left(Z_{j}\right)$ by $E, \sigma^{2}$ and $\phi_{i}\left(F^{-1}\left(U_{j: N}\right)\right)=\Psi_{i}\left(U_{j: N}\right)$ without changing anything. Next, arguing as in Corollary A2.2 of ABZ (1976), we see that for all sums of the form $\sum a_{j}{ }^{k}$ and $\sum a_{j}{ }^{k} E h\left(U_{j: N}\right)$ occurring in $\tilde{\alpha}_{0}, \ldots, \tilde{\alpha}_{5}, \tilde{\beta}_{0}, \ldots, \tilde{\beta}_{5}$ we may write

$$
\begin{gathered}
\frac{1}{N} \sum a_{j}{ }^{k}=\int J^{k}(t) d t+o(1), \\
\frac{1}{N} \sum a_{j}{ }^{k} E h\left(U_{j: N}\right)=\int J^{k}(t) h(t) d t+o(1),
\end{gathered}
$$

and also

$$
\frac{1}{N} \sigma^{2}\left(\sum a_{j} \Psi_{1}\left(U_{j: N}\right)\right)=\iint J(s) J(t) \Psi_{1}{ }^{\prime}(s) \Psi_{1}{ }^{\prime}(t)[s \wedge t-s t] d s d t+o(1) .
$$

We note that $\bar{\alpha}_{0}, \ldots, \bar{\alpha}_{5}, \bar{\beta}_{0}, \ldots, \bar{\beta}_{5}$ are obtained from $\tilde{\alpha}_{0}, \ldots, \tilde{\alpha}_{5}, \tilde{\beta}_{0}, \ldots, \tilde{\beta}_{5}$ by replacing every expression of the form $(5.20)-(5.22)$ by the corresponding integral on the right in $(5.20)-(5.22)$. Since $\int J^{2}(t) d t>0$, we know that for those terms in $\tilde{\alpha}_{0}, \ldots, \tilde{\alpha}_{5}, \tilde{\beta}_{0}, \ldots, \tilde{\beta}_{5}$ that are $O\left(N^{-1}\right)$, this substitution can only introduce errors that are $o\left(N^{-1}\right)$.

The first terms in $\tilde{\alpha}_{0}, \tilde{\alpha}_{1}$ and $\tilde{\alpha}_{2}$ as well as the second term in $\tilde{\beta}_{0}$ are generally not $O\left(N^{-1}\right)$ but only $O\left(N^{-\frac{1}{2}}\right)$, and here the substitution of integrals for sums gives rise to more complicated remainder terms. This creates problems we did not encounter in the one-sample case where certain symmetries prohibit the occurrence of $O\left(N^{-\frac{1}{2}}\right)$ terms. We have

$$
\begin{gathered}
\frac{1}{N} \sum a_{j}{ }^{2}=\int J^{2}(t) d t-\frac{1}{N} \sum \sigma^{2}\left(J\left(U_{j: N}\right)\right), \\
\frac{1}{N} \sum a_{j}{ }^{3}=\int J^{3}(t) d t-\frac{1}{N} \sum \operatorname{Cov}\left(J\left(U_{j: N}\right), J^{2}\left(U_{j: N}\right)\right)-\frac{1}{N} \sum E J\left(U_{j: N}\right) \sigma^{2}\left(J\left(U_{j: N}\right)\right), \\
\frac{1}{N} \sum a_{j} E \Psi_{2}\left(U_{j: N}\right)=\int J(t) \Psi_{2}(t) d t-\frac{1}{N} \sum \operatorname{Cov}\left(J\left(U_{j: N}\right), \Psi_{2}\left(U_{j: N}\right)\right) \\
\frac{1}{N} \sum a_{j}{ }^{2} E \Psi_{1}\left(U_{j: N}\right)=\int J^{2}(t) \Psi_{1}(t) d t-\frac{1}{N} \sum \operatorname{Cov}\left(J^{2}\left(U_{j: N}\right), \Psi_{1}\left(U_{j: N}\right)\right) \\
-\frac{1}{N} \sum E \Psi_{1}\left(U_{j: N}\right) \sigma^{2}\left(J\left(U_{j: N}\right)\right) .
\end{gathered}
$$

By (A2.22) in $\mathrm{ABZ}(1976), N^{-\frac{3}{2}} \sum \sigma^{2}\left(J\left(U_{j: N}\right)\right)=o\left(N^{-1}\right)$. It follows that for $k=0, \cdots, 5$,

$$
\begin{aligned}
\tilde{\alpha}_{k}-\tilde{\alpha}_{k} & =o\left(N^{-1}\right)+O\left(M_{1}\right), \quad \tilde{\beta}_{k}-\bar{\beta}_{k}=o\left(N^{-1}\right)+O\left(M_{1}\right), \\
M_{1}=(1 & -2 \lambda) N^{-\frac{3}{2}}\left[\left|\sum \operatorname{Cov}\left(J\left(U_{j: N}\right), J^{2}\left(U_{j: N}\right)\right)\right|\right. \\
& +\left|\sum E J\left(U_{j: N}\right) \sigma^{2}\left(J\left(U_{j: N}\right)\right)\right|+\left|\sum \operatorname{Cov}\left(J\left(U_{j: N}\right), \Psi_{2}\left(U_{j: N}\right)\right)\right| \\
& \left.+\left|\sum \operatorname{Cov}\left(J^{2}\left(U_{j: N}\right), \Psi_{1}\left(U_{j: N}\right)\right)\right|+\left|\sum E \Psi_{1}\left(U_{j: N}\right) \sigma^{2}\left(J\left(U_{j: N}\right)\right)\right|\right] .
\end{aligned}
$$


By (A2.17), (A2.22) and (A2.23) in ABZ (1976) we have

$$
\begin{aligned}
\eta= & \bar{\eta} \\
& \frac{1}{2}\left(\frac{\lambda(1-\lambda)}{N \int J^{2}(t) d t}\right)^{\frac{1}{2}} \theta\left[2 \sum \operatorname{Cov}\left(J\left(U_{j: N}\right), \Psi_{1}\left(U_{j: N}\right)\right)\right. \\
& \left.\quad-\frac{\int J(t) \Psi_{1}(t) d t}{\int J^{2}(t) d t} \sum \sigma^{2}\left(J\left(U_{j: N}\right)\right)\right]+o\left(N^{-1}\right) \\
= & \bar{\eta}+o\left(N^{-\frac{1}{2}}\right) .
\end{aligned}
$$

Hence, uniformly in $x$,

$$
\begin{aligned}
\tilde{K}(x-\eta)= & \Phi(x-\bar{\eta})-\phi(x-\bar{\eta})\left[(\eta-\bar{\eta})+\sum_{k=0}^{5} \bar{\alpha}_{k} H_{k}(x-\bar{\eta})\right] \\
& \quad+o\left(N^{-1}\right)+O\left(M_{1}\right) \\
= & \bar{K}_{1}(x-\bar{\eta})+o\left(N^{-1}\right)+O\left(M_{1}\right)
\end{aligned}
$$

and similarly

$$
\tilde{\pi}(F, \theta)=\bar{\pi}_{1}(F, \theta)+o\left(N^{-1}\right)+O\left(M_{1}\right) .
$$

It follows that, in order to prove (5.14) and (5.16), it suffices to show that $M_{1}=o\left(N^{-1}\right)$. Since (5.15) and (5.17) are immediate consequences of (5.14) and (5.16) on the one hand and (A2.22) and (A2.23) in ABZ (1976) on the other, the proof of the theorem will then be complete.

At this point we finally need condition (5.4) rather than the weaker assumption $\int J^{4}(t) d t<\infty$. Using (5.4), (5.18) and (5.19) and proceeding as in the proof of Corollary A2.1 in ABZ (1976), we find that each term of $M_{1}$ is

$$
o\left(N^{-\frac{3}{2}} \int_{N^{-1}}^{1-N^{-1}}\{t(1-t)\}^{-\frac{3}{2}} d t\right)=o\left(N^{-1}\right) .
$$

REMARK. In the above we have stressed the fact that the only reason for requiring (5.4) rather than assuming $\int J^{4}(t) d t<\infty$ is that we have to show that $M_{1}=o\left(N^{-1}\right)$. However, there are special cases of interest where $\int J^{4}(t) d t<\infty$ suffices. If either $\lambda=\frac{1}{2}$, or $f$ is a symmetric density and $J(t)$ is antisymmetric about $t=\frac{1}{2}$, then $M_{1}=0$. Less trivially, since $\int J^{4}(t) d t<\infty$ and (5.5) imply that $J^{\prime}(t)=o\left(\{t(1-t)\}^{-\frac{5}{4}}\right)$, we can follow the reasoning leading to (5.26) while retaining the factor $(1-2 \lambda)$, to arrive at

$$
M_{1}=o\left(|1-2 \lambda| N^{-\frac{3}{2}} \int_{N^{-1}}^{1-1}\{t(1-t)\}^{-\frac{7}{4}} d t\right)=o\left(|1-2 \lambda| N^{-\frac{3}{4}}\right) .
$$

Hence in the special cases where either $\lambda=\frac{1}{2}+O\left(N^{-\frac{1}{4}}\right)$, or $f$ is a symmetric density and $J$ is antisymmetric about the point $\frac{1}{2}$, the conclusions of Theorem 5.1 will hold if condition (5.4) is replaced by the assumption $\int J^{4}(t) d t<\infty$. Comparison with $\mathrm{ABZ}$ (1976) shows that in these special cases the conditions under which Theorem 5.1 holds are essentially the same as the conditions of the comparable Theorem 4.1 in ABZ (1976) for the one-sample problem. This is not surprising as one may think of the one-sample case under contiguous alternatives as a two-sample situation with $\lambda=\frac{1}{2}+O_{P}\left(N^{-\frac{1}{2}}\right)$.

We now turn to the special case $J=-\Psi_{1}$. For $F \in \widetilde{F}$ we obtain by partial 
integration

$$
\begin{aligned}
& \int \Psi_{1}(t) \Psi_{2}(t) d t=\frac{1}{2} \int \Psi_{1}{ }^{3}(t) d t \\
& \int \Psi_{1}{ }^{2}(t) \Psi_{2}(t) d t=\frac{2}{3} \int \Psi_{1}{ }^{4}(t) d t \\
& \int \Psi_{1}(t) \Psi_{3}(t) d t=\frac{2}{3} \int \Psi_{1}{ }^{4}(t) d t-\int \Psi_{2}{ }^{2}(t) d t \\
& \iint \Psi_{1}(s) \Psi_{1}(t) \Psi_{1}{ }^{\prime}(s) \Psi_{1}{ }^{\prime}(t)[s \wedge t-s t] d s d t=\frac{1}{4} \int \Psi_{1}{ }^{4}(t) d t-\frac{1}{4}\left(\int \Psi_{1}{ }^{2}(t) d t\right)^{2}
\end{aligned}
$$

Substitution of $J=-\Psi_{1}$ and application of (5.28) considerably simplifies the expressions (5.8) and (5.12) for $\bar{\alpha}_{k}$ and $\bar{\beta}_{k}$. Note that $\bar{\eta}$ defined by (5.11) reduces to

$$
\eta^{*}=\theta\left\{\lambda(1-\lambda) N \int \Psi_{1}^{2}(t) d t\right\}^{\frac{1}{2}}
$$

The expressions for $\bar{\alpha}_{k}$ and $\bar{\beta}_{k}$ simplify somewhat further if we express $\theta$ in terms of $\eta^{*}$ throughout. Finally we rearrange the terms in $\sum \bar{\alpha}_{k} H_{k}\left(x-\eta^{*}\right)$ and $\sum \bar{\beta}_{k} H_{k}\left(u_{\alpha}-\eta^{*}\right)$ according to the integrals involved and substitute the explicit expressions (2.43) for the Hermite polynomials $H_{k}$. In this way we find after laborious but straightforward calculations that for $J=-\Psi_{1}$,

$$
\begin{aligned}
& \Phi(x-\bar{\eta})-\phi(x-\bar{\eta}) \sum_{k=0}^{5} \bar{\alpha}_{k} H_{k}(x-\bar{\eta})=L_{0}(x), \\
& 1-\Phi\left(u_{\alpha}-\bar{\eta}\right)+\phi\left(u_{\alpha}-\bar{\eta}\right) \sum_{k=0}^{5} \bar{\beta}_{k} H_{k}\left(u_{\alpha}-\bar{\eta}\right)=\pi_{0}^{*}(F, \theta),
\end{aligned}
$$

where

$$
\begin{aligned}
& L_{0}(x)=\Phi\left(x-\eta^{*}\right) \\
& -\frac{\phi\left(x-\eta^{*}\right)}{288}\left[\frac{24(1-2 \lambda)}{\{\lambda(1-\lambda) N\}^{\frac{1}{2}}} \frac{\int \Psi_{1}^{3}(t) d t}{\left\{\int \Psi_{1}^{2}(t) d t\right\}^{\frac{3}{2}}}\right. \\
& \times\left\{-2\left(x^{2}-1\right)-2 \eta^{*} x+\eta^{* 2}\right\}+\frac{4}{\lambda(1-\lambda) N} \frac{\int \Psi_{1}^{4}(t) d t}{\left\{\int \Psi_{1}^{2}(t) d t\right\}^{2}} \\
& \times\left\{3\left(1-6 \lambda+6 \lambda^{2}\right)\left(x^{3}-3 x+\eta^{*}\left(x^{2}-1\right)\right)-3\left(1-5 \lambda+5 \lambda^{2}\right) \eta^{* 2} x\right. \\
& \left.+5\left(1-3 \lambda+3 \lambda^{2}\right) \eta^{* 3}\right\}-\frac{48}{\lambda(1-\lambda) N} \frac{\int \Psi_{2}^{2}(t) d t}{\left\{\int \Psi_{1}^{2}(t) d t\right\}^{2}} \\
& \times\left(1-3 \lambda+3 \lambda^{2}\right) \eta^{* 3}+\frac{(1-2 \lambda)^{2}}{\lambda(1-\lambda) N} \frac{\left\{\int \Psi_{1}{ }^{3}(t) d t\right\}^{2}}{\left\{\int \Psi_{1}{ }^{2}(t) d t\right\}^{3}} \\
& \times\left\{4\left(x^{5}-10 x^{3}+15 x\right)+4 \eta^{*}\left(x^{4}-6 x^{2}+3\right)-8 \eta^{* 2}\left(x^{3}-3 x\right)\right. \\
& \left.-4 \eta^{* 3}\left(x^{2}-1\right)+5 \eta^{* 4} x-\eta^{* 5}\right\}+\frac{144 x}{N}+\frac{36}{\lambda(1-\lambda) N} \\
& \times\left\{-(1-2 \lambda)^{2}\left(x^{3}-3 x+\eta^{*} x^{2}\right)+\eta^{*}+\left(1-5 \lambda+5 \lambda^{2}\right) \eta^{* 2} x\right. \\
& \left.\left.+\left(1-3 \lambda+3 \lambda^{2}\right) \eta^{* 3}\right\}\right] \\
& \pi_{0}^{*}(F, \theta)=1-\Phi\left(u_{\alpha}-\eta^{*}\right) \\
& +\frac{\eta^{*} \phi\left(u_{\alpha}-\eta^{*}\right)}{288}\left[\frac{24(1-2 \lambda)}{\{\lambda(1-\lambda) N\}^{\frac{1}{2}}} \frac{\int \Psi_{1}^{3}(t) d t}{\left\{\int \Psi_{1}^{2}(t) d t\right\}^{\frac{3}{2}}}\left(-2 u_{\alpha}+\eta^{*}\right)\right. \\
& +\frac{4}{\lambda(1-\lambda) N} \frac{\int \Psi_{1}^{4}(t) d t}{\left\{\int \Psi_{1}^{2}(t) d t\right\}^{2}}\left\{3\left(1-6 \lambda+6 \lambda^{2}\right)\left(u_{\alpha}^{2}-1\right)\right.
\end{aligned}
$$


Define

$$
\begin{aligned}
& \left.-3\left(1-5 \lambda+5 \lambda^{2}\right) \eta^{*} u_{\alpha}+5\left(1-3 \lambda+3 \lambda^{2}\right) \eta^{* 2}\right\} \\
& -\frac{48}{\lambda(1-\lambda) N} \frac{\int \Psi_{2}^{2}(t) d t}{\left\{\int \Psi_{1}^{2}(t) d t\right\}^{2}}\left(1-3 \lambda+3 \lambda^{2}\right) \eta^{* 2} \\
& +\frac{(1-2 \lambda)^{2}}{\lambda(1-\lambda) N} \frac{\left\{\int \Psi_{1}{ }^{3}(t) d t\right\}^{2}}{\left\{\int \Psi_{1}^{2}(t) d t\right\}^{3}}\left\{-8\left(2 u_{\alpha}{ }^{2}-1\right)\right. \\
& \left.+4 \eta^{*}\left(u_{\alpha}^{3}+3 u_{\alpha}\right)-8 \eta^{* 2}\left(u_{\alpha}^{2}-1\right)+5 \eta^{* 3} u_{\alpha}-\eta^{* 4}\right\} \\
& +\frac{36}{\lambda(1-\lambda) N}\left\{-(1-2 \lambda)^{2} u_{\alpha}^{2}+1+\left(1-5 \lambda+5 \lambda^{2}\right) \eta^{*} u_{\alpha}\right. \\
& \left.+\left(1-3 \lambda+3 \lambda^{2}\right) \eta^{* 2}\right\} .
\end{aligned}
$$

$$
\begin{aligned}
& L_{1}(x)=L_{0}(x)+\frac{\eta^{*} \phi\left(x-\eta^{*}\right)}{2 N \int \Psi_{1}^{2}(t) d t} \sum_{j=1}^{v} \sigma^{2}\left(\Psi_{1}\left(U_{j: N}\right)\right) \\
& L_{2}(x)=L_{0}(x)+\frac{\eta^{*} \phi\left(x-\eta^{*}\right)}{2 N \int \Psi_{1}^{2}(t) d t} \int_{N^{-1}}^{1-N^{-1}}\left(\Psi_{1}^{\prime}(t)\right)^{2} t(1-t) d t \\
& L_{3}(x)=L_{0}(x)+\frac{\eta^{*} \phi\left(x-\eta^{*}\right)}{2 N \int \Psi_{1}^{2}(t) d t} \sum_{j=1}^{v} E\left(\Psi_{1}\left(U_{j: N}\right)-\Psi_{1}\left(\frac{j}{N+1}\right)\right)^{2}, \\
& \pi_{1}^{*}(F, \theta)=\pi_{0}^{*}(F, \theta)-\frac{\eta^{*} \phi\left(u_{\alpha}-\eta^{*}\right)}{2 N \int \Psi_{1}^{2}(t) d t} \sum_{j=1}^{N} \sigma^{2}\left(\Psi_{1}\left(U_{j: N}\right)\right) \\
& \pi_{2}^{*}(F, \theta)=\pi_{0}^{*}(F, \theta)-\frac{\eta^{*} \phi\left(u_{\alpha}-\eta^{*}\right)}{2 N \int \Psi_{1}^{2}(t) d t} \int_{N^{-1}}^{1-N-1}\left(\Psi_{1}{ }^{2}(t)\right)^{2} t(1-t) d t \\
& \pi_{3}^{*}(F, \theta)=\pi_{0}^{*}(F, \theta) \\
& \quad-\frac{\eta^{*} \phi\left(u_{\alpha}-\eta^{*}\right)}{2 N \int \Psi_{1}^{2}(t) d t} \sum_{j=1}^{y} E\left(\Psi_{1}\left(U_{j: N}\right)-\Psi_{1}\left(\frac{j}{N+1}\right)\right)^{2} .
\end{aligned}
$$

Note that (5.9), (5.10), (5.11), (5.13), (5.30) and (5.31) imply that for $J=-\Psi_{1}$, $\bar{K}_{i}(x-\bar{\eta})=L_{i}(x)$ and $\bar{\pi}_{i}(F, \theta)=\pi_{i}^{*}(F, \theta)$ for $i=1,2$. The expansions $L_{3}$ and $\pi_{3}^{*}$ are connected only with approximate scores that were not considered so far.

THEOREM 5.2. Let $F \in \mathscr{T}, J=-\Psi_{1}, G(x)=F(x-\theta), 0 \leqq \theta \leqq D N^{-\frac{1}{2}}, \varepsilon \leqq$ $\lambda \leqq 1-\varepsilon$ and $\varepsilon^{\prime} \leqq \alpha \leqq 1-\varepsilon^{\prime}$ for positive $D, \varepsilon$ and $\varepsilon^{\prime}$. Then, for every fixed $F$, $D, \varepsilon$ and $\varepsilon^{\prime}$, there exist positive numbers $B, \delta_{1}, \delta_{2}, \cdots$ with $\lim _{N \rightarrow \infty} \delta_{N}=0$ such that the following statements hold for every $N$.

(i) For exact scores $a_{j}=-E \Psi_{1}\left(U_{j: N}\right)$,

$$
\sup _{x}\left|P\left(\frac{T}{\left\{\lambda(1-\lambda) \sum a_{j}^{2}\right\}^{\frac{1}{2}}} \leqq x\right)-L_{1}(x)\right| \leqq \delta_{N} N^{-1},
$$

$$
\begin{aligned}
\sup _{x}\left|P\left(\frac{T}{\left\{\lambda(1-\lambda) \sum a_{j}{ }^{2}\right\}^{\frac{1}{2}}} \leqq x\right)-L_{2}(x)\right| \\
\quad \leqq \delta_{N} N^{-1}+B N^{-\frac{3}{2}} \int_{N^{-1}}^{1-N^{-1}}\left(\Psi_{1}^{\prime}(t)\right)^{2}\{t(1-t)\}^{\frac{1}{2}} d t,
\end{aligned}
$$

(5.36) $\left|\pi(F, \theta)-\pi_{2}^{*}(F, \theta)\right| \leqq \delta_{N} N^{-1}+B N^{-\frac{3}{2}} \int_{N^{-1}}^{1-N^{-1}}\left(\Psi_{1}^{\prime}(t)\right)^{2}\{t(1-t)\}^{\frac{1}{2}} d t$; 
(ii) For approximate scores $a_{j}=-\Psi_{1}(j /(N+1))$,

$$
\begin{gathered}
\sup _{x}\left|P\left(\frac{T-\lambda \sum a_{j}}{\left\{\lambda(1-\lambda) \sum a_{j}^{2}\right\}^{\frac{1}{2}}} \leqq x\right)-L_{3}(x)\right| \leqq \delta_{N} N^{-1} \\
\sup _{x}\left|P\left(\frac{T-\lambda \sum a_{j}}{\left\{\lambda(1-\lambda) \sum a_{j}{ }^{2}\right\}^{\frac{1}{2}}} \leqq x\right)-L_{2}(x)\right| \\
\leqq \delta_{N} N^{-1}+B N^{-\frac{3}{2}} \int_{N^{-1}}^{1-N^{-1}}\left(\Psi_{1}{ }^{\prime}(t)\right)^{2}\{t(1-t)\}^{\frac{1}{2}} d t \\
\left|\pi(F, \theta)-\pi_{3}{ }^{*}(F, \theta)\right| \leqq \delta_{N} N^{-1}
\end{gathered}
$$

and (5.36) continues to hold.

Proof. For $F \in \mathscr{T}, \Psi_{1}$ is not constant on $(0,1), \int \Psi_{1}(t) d t=0$ and $\Psi_{1}{ }^{6}$ is summable. In view of the remark following Definition 5.1, this implies that $J \in \mathscr{J}$. We have already noted that $K_{i}(x-\eta)=L_{i}(x)$ and $\pi_{i}(F, \theta)=\pi_{i}{ }^{*}(F, \theta)$ for $i=$ 1,2 , if $J=-\Psi_{1}$. Part (i) of the theorem is therefore an immediate consequence of Theorem 5.1.

To prove part (ii) we retrace the proof of Theorem 5.1 for $J=-\Psi_{1}$ and approximate scores $a_{j}=-\Psi_{1}(j /(N+1))$. The first difficulty we encounter is that in general $\sum a_{j} \neq 0$. However, Lemma A2.3 of ABZ (1976), (5.7) and (5.18) yield

$$
a_{.}=\frac{1}{N} \sum_{j=1}^{y} a_{j}=-\int_{0}^{1} \Psi_{1}(t) d t+O\left(N^{-1} \int_{N^{-1}}^{1-N^{-1}}\left|\Psi_{1}^{\prime}(t)\right| d t\right)=o\left(N^{-\frac{5}{6}}\right)
$$

and one easily verifies that the conditions of Theorem 4.1 hold for the reduced scores $a_{j}-a_{.}$. Since the assumptions of Theorem 4.2 are also satisfied, we have

$$
\sup _{x}\left|P\left(\frac{T-\lambda \sum a_{j}}{\left\{\lambda(1-\lambda) \sum\left(a_{j}-a_{0}\right)^{2}\right\}^{\frac{1}{2}}} \leqq x\right)-\hat{K}(x-\hat{\eta})\right|=O\left(N^{-\frac{5}{4}}\right),
$$

where $\hat{K}$ and $\hat{\eta}$ are obtained from $\tilde{K}$ and $\eta$ by replacing $a_{j}$ by $a_{j}-a_{\bullet}$ throughout. Because, by (3.10) and (5.40),

$$
\sum\left(a_{j}-a_{0}\right)^{2}=\sum a_{j}{ }^{2}\left(1+o\left(N^{-\frac{5}{3}}\right)\right),
$$

we can change the norming constant $\sum\left(a_{j}-a_{0}\right)^{2}$ of $T$ in $(5.41)$ back to $\sum a_{j}{ }^{2}$ with impunity. As $\int \Psi_{1}(t) d t=0,(5.42)$ also ensures that $|\hat{\eta}-\eta|=o\left(N^{-\frac{5}{3}}\right)$. Finally (A2.16) of ABZ (1976) and (5.18) imply that $\sigma_{F}{ }^{2}\left(\sum a_{j} \psi_{1}\left(Z_{j}\right)\right)=O(N)$ for $J=-\Psi_{1}$ and, together with (5.42), (3.10), (5.6) and (5.40), this yields $\sup _{x}|\hat{K}(x)-\tilde{K}(x)|=o\left(N^{-\frac{4}{3}}\right)$. Combining these results we find

$$
\sup _{x}\left|P\left(\frac{T-\lambda \sum a_{j}}{\left\{\lambda(1-\lambda) \sum a_{j}^{2}\right\}^{\frac{1}{2}}} \leqq x\right)-\tilde{K}(x-\eta)\right|=O\left(N^{-\frac{5}{4}}\right)
$$

and similarly

$$
|\pi(F, \theta)-\tilde{\pi}(F, \theta)|=O\left(N^{-\frac{5}{4}}\right) .
$$

The remainder of the proof parallels that of Theorem 5.1 for the special case $J=-\Psi_{1}$. We replace all sums as well as $\sigma^{2}\left(\sum a_{j} \Psi_{1}\left(U_{j: N}\right)\right)$ by the appropriate 
integrals. The reasoning of Corollary A2.2 of ABZ (1976) shows that for those terms in the expansions that are $O\left(N^{-1}\right)$, this substitution will only lead to errors that are $o\left(N^{-1}\right)$. For the $O\left(N^{-\frac{1}{2}}\right)$ terms the error committed is $O\left(M_{1}\right)+O\left(M_{2}\right)$, where $M_{1}$ is given by (5.24) with $J=-\Psi_{1}$ and $M_{2}$ originates from the difference between exact and approximate scores. It was shown in the proof of Theorem 5.1 that $M_{1}=o\left(N^{-1}\right)$. With regard to $M_{2},(5.7)$, Lemma A2.3 of ABZ (1976), (5.18) and (5.19) imply that, uniformly in $j$,

$$
\begin{aligned}
& \left|\left\{E \Psi_{1}\left(U_{j: N}\right)\right\}^{k}-\Psi_{1}{ }^{k}\left(\frac{j}{N+1}\right)\right| \\
& =O\left(N^{-1}\right)+o\left(N^{-1}\left\{\frac{j(N-j+1)}{(N+1)^{2}}\right\}^{-1-k / 6}\right) \\
& \left|E \Psi_{1}\left(U_{j: N}\right)\right|=O(1)+o\left(\left\{\frac{j(N-j+1)}{(N+1)^{2}}\right\}^{-\frac{1}{6}}\right) \\
& \left|E \Psi_{2}\left(U_{j: N}\right)\right|=O(1)+o\left(\left\{\frac{j(N-j+1)}{(N+1)^{2}}\right\}^{-\frac{1}{3}}\right)
\end{aligned}
$$

where $k=1,2,3$. It follows that $M_{2}$ is of the form (5.26) and is therefore $o\left(N^{-1}\right)$.

It remains to replace $\eta$ by $\eta^{*}$. Because of (5.7), (5.18) and Lemma A2.3 of $\operatorname{ABZ}(1976), N^{-1} \sum \sigma^{2}\left(\Psi_{1}\left(U_{j: N}\right)\right)=o\left(N^{-\frac{2}{3}}\right)$, and in view of $(5.45)$,

$$
\begin{aligned}
& \frac{1}{N} \sum \Psi_{1}\left(\frac{j}{N+1}\right) E \Psi_{1}\left(U_{j: N}\right)-\int \Psi_{1}{ }^{2}(t) d t \\
&=-\frac{1}{N} \sum E \Psi_{1}\left(U_{j: N}\right)\left[\Psi_{1}\left(U_{j: N}\right)-\Psi_{1}\left(\frac{j}{N+1}\right)\right]=o\left(N^{-\frac{2}{3}}\right) \\
& \frac{1}{N} \sum \Psi_{1}^{2}\left(\frac{j}{N+1}\right)-\int \Psi_{1}^{2}(t) d t=-\frac{1}{N} \sum\left[E \Psi_{1}{ }^{2}\left(U_{j: N}\right)-\Psi_{1}{ }^{2}\left(\frac{j}{N+1}\right)\right] \\
&=o\left(N^{-\frac{2}{3}}\right) .
\end{aligned}
$$

Hence, for $J=-\Psi_{1}$,

$$
\begin{aligned}
\eta & =\eta^{*}-\frac{\eta^{*}}{2 N \int \Psi_{1}^{2}(t) d t} \sum E\left\{\Psi_{1}\left(U_{j: N}\right)-\Psi_{1}\left(\frac{j}{N+1}\right)\right\}^{2}+o\left(N^{-\frac{f}{3}}\right) \\
& =\eta^{*}+o\left(N^{-\frac{2}{3}}\right)
\end{aligned}
$$

and a comparison with (5.25) for $J=-\Psi_{1}$ show that (5.37) and (5.39) will hold if $L_{3}$ and $\pi_{3}^{*}$ can be obtained from $L_{1}$ and $\pi_{1}^{*}$ by replacing $\sum \sigma^{2}\left(\Psi_{1}\left(U_{j: N}\right)\right)$ by $\sum E\left\{\Psi_{1}\left(U_{j: N}\right)-\Psi_{1}(j /(N+1))\right\}^{2}$. Since this is true, (5.37) and (5.39) are proved. The validity of (5.38) and (5.36) for approximate scores is a consequence of (5.37), (5.39) and Corollary A2.2 of ABZ (1976). The proof of the theorem is complete.

At this point it is appropriate to repeat some remarks made in ABZ (1976). The correspondence between expansions (5.34) and (5.38) and the fact that 
(5.36) holds for both exact and approximate scores seem to be typical for the case $J=-\Psi_{1}$. In the general case where $J \neq-\Psi_{1}$, expansions (5.15) and (5.17) will not hold for approximate scores even if $T$ is replaced by $T-\lambda \sum a_{j}$ in (5.15). A second remark is that the growth conditions on $J^{\prime}$ and $\Psi_{1}^{\prime}$ implicit in our assumptions (viz. (5.4) and (5.18)) do not guarantee that the right-hand side in (5.15), (5.17), (5.34), (5.36) and (5.38) is indeed $o\left(N^{-1}\right)$ as is our aim. For this we would need $J^{\prime}(t)=o\left(\{t(1-t)\}^{-1}\right)$ and $\Psi_{1}^{\prime}(t)=o\left(\{t(1-t)\}^{-1}\right)$. This may explain the presence of the remaining expansions in Theorems 5.1 and 5.2, which are less explicit but do have remainder $o\left(N^{-1}\right)$ under the conditions stated. Note that their presence in Theorem 5.2 also indicates that even for $J=-\Psi_{1}$, expansions for exact and approximate scores are not necessarily identical to $o\left(N^{-1}\right)$. Finally we should point out that similar expansions with remainder $o\left(N^{-1}\right)$ might have been given in Theorem 4.2 of $\mathrm{ABZ}(1976)$ where they were unfortunately omitted.

We conclude this section with a few examples of the power expansions in Theorems 5.1 and 5.2. First we consider the powers $\pi_{W}(\Phi, \theta)$ and $\pi_{W}(\Lambda, \theta)$ of Wilcoxon's two-sample test $(W)$ against normal and logistic location alternatives $(\Phi(x), \Phi(x-\theta))$ and $(\Lambda(x), \Lambda(x-\theta))$ respectively, where $\Lambda(x)=$ $(1+\exp \{-x\})^{-1}$ and $\theta=O\left(N^{-\frac{1}{2}}\right)$. We find

$$
\begin{aligned}
\pi_{W}(\Phi, \theta)=1 & -\Phi\left(u_{\alpha}-\bar{\eta}\right)+\frac{\bar{\eta} \phi\left(u_{\alpha}-\bar{\eta}\right)}{N} \\
& \times\left[-\frac{1}{2}-\frac{37-217 \lambda+217 \lambda^{2}}{20 \lambda(1-\lambda)}\left(u_{\alpha}^{2}-1\right)\right. \\
& +\frac{1}{\lambda(1-\lambda)}\left\{\frac{3^{\frac{1}{2}}}{6}+\frac{67-437 \lambda+437 \lambda^{2}}{20}\right\} u_{\alpha} \bar{\eta} \\
& -\frac{1}{\lambda(1-\lambda)}\left\{\frac{3^{\frac{1}{2}}}{6}+\frac{\pi}{36}+\frac{29-219 \lambda+219 \lambda^{2}}{20}\right\} \bar{\eta}^{2} \\
& \left.+\frac{\left(1-6 \lambda+6 \lambda^{2}\right)}{\lambda(1-\lambda)} \frac{6 \arctan 2^{\frac{1}{2}}}{\pi}\left\{u_{\alpha}{ }^{2}-1-2 u_{\alpha} \bar{\eta}+\bar{\eta}^{2}\right\}\right] \\
& +o\left(N^{-1}\right),
\end{aligned}
$$

where $\bar{\eta}=(3 \lambda(1-\lambda) N / \pi)^{\frac{1}{2}} \theta$, and

$$
\begin{aligned}
\pi_{W}(\Lambda, \theta)=1 & -\Phi\left(u_{\alpha}-\eta^{*}\right)+\frac{\eta^{*} \phi\left(u_{\alpha}-\eta^{*}\right)}{N} \\
& \times\left[-\frac{1}{2}-\frac{1-\lambda+\lambda^{2}}{20 \lambda(1-\lambda)}\left(u_{\alpha}^{2}-1\right)+\frac{1-5 \lambda+5 \lambda^{2}}{20 \lambda(1-\lambda)} u_{\alpha} \eta^{*}\right. \\
& \left.-\frac{1-3 \lambda+3 \lambda^{2}}{20 \lambda(1-\lambda)} \eta^{* 2}\right]+o\left(N^{-1}\right)
\end{aligned}
$$

where $\eta^{*}=(\lambda(1-\lambda) N / 3)^{\frac{1}{2}} \theta$.

As a second example we compute expansions for the powers $\pi_{N S}(\Phi, \theta)$ and $\pi_{N S}(\Lambda, \theta)$ of the two-sample normal scores test against the normal and logistic 
location alternatives described above. One of the integrals occurring in this computation is

$$
\int_{N^{-1}}^{1-N^{-1}} \frac{t(1-t)}{\left\{\phi\left(\Phi^{-1}(t)\right)\right\}^{2}} d t=2 \int_{0}^{\Phi^{-1}\left(1-N^{-1}\right)} \frac{\Phi(x)(1-\Phi(x))}{\phi(x)} d x
$$

and since its asymptotic evaluation is not entirely trivial, we provide some details. Let $\gamma$ denote Euler's constant

$$
\gamma=\lim _{k \rightarrow \infty}\left\{\sum_{j=1}^{k} \frac{1}{j}-\log k\right\}=0.577216 \ldots
$$

and note that (cf. Ryshik and Gradstein (1957), page 197)

$$
\int_{0}^{\infty} e^{-u} \log u d u=-\gamma,
$$

$$
\int_{0}^{\infty} \phi(u) \log u d u=-\frac{1}{4} \log 2-\frac{1}{4} \gamma .
$$

To evaluate (5.49) we begin by writing for $z>0$

$$
\begin{aligned}
\int_{0}^{z} \frac{1-\Phi(x)}{\phi(x)} d x & =\int_{0}^{z} d x \int_{x}^{\infty} e^{-\frac{1}{2}(y-x)(y+x)} d y=\frac{1}{2} \int_{0}^{\infty} d u \int_{u}^{2 z+u} e^{-\frac{1}{2} u v} d v \\
& =\int_{0}^{\infty} \frac{1}{u} e^{-\frac{1}{2} u^{2}}\left(1-e^{-z u}\right) d u .
\end{aligned}
$$

It follows from (5.53) and (5.51) that for $z \rightarrow \infty$,

$$
\begin{aligned}
\int_{0}^{z} \frac{1-\Phi(x)}{\phi(x)} d x= & \int_{0}^{z^{-\frac{1}{2}}} \frac{1}{u}\left(1-e^{-z u}\right) d u+\int_{z^{-\frac{1}{2}}}^{\infty} \frac{1}{u} e^{-\frac{1}{2} u^{2}} d u+o(1) \\
= & \int_{0}^{z^{\frac{1}{2}}} \frac{1}{u}\left(1-e^{-u}\right) d u+\frac{1}{2} \int_{(2 z)-1}^{\infty} \frac{1}{u} e^{-u} d u+o(1) \\
= & \frac{1}{2} \log z-\int_{0}^{z^{\frac{1}{2}}} e^{-u} \log u d u+\frac{1}{2} \log (2 z) \\
& \quad+\frac{1}{2} \int_{(2 z)-1}^{\infty} e^{-u} \log u d u+o(1) \\
= & \log z+\frac{1}{2} \log 2+\frac{1}{2} \gamma+o(1) .
\end{aligned}
$$

Similarly (5.53), (5.51) and (5.52) imply that

$$
\begin{aligned}
\int_{0}^{\infty} \frac{(1-\Phi(x))^{2}}{\phi(x)} d x & =\int_{0}^{\infty} \phi(x) d x \int_{0}^{\infty} \frac{1}{u} e^{-\frac{1}{2} u^{2}}\left(1-e^{-x u}\right) d u \\
& =\int_{0}^{\infty} \frac{1}{u}\left\{\frac{1}{2} e^{-\frac{1}{2} u^{2}}-(1-\Phi(u))\right\} d u \\
& =\int_{0}^{\infty} \log u\left\{\frac{1}{2} u e^{-\frac{1}{2} u^{2}}-\phi(u)\right\} d u \\
& =\frac{1}{4} \log 2+\frac{1}{4} \int_{0}^{\infty} e^{-x} \log x d x-\int_{0}^{\infty} \phi(u) \log u d u \\
& =\frac{1}{2} \log 2 .
\end{aligned}
$$

Since $\log \Phi^{-1}\left(1-N^{-1}\right)=\frac{1}{2} \log \log N+\frac{1}{2} \log 2+o(1)$ for $N \rightarrow \infty,(5.49),(5.54)$ and (5.55) imply that

$$
\int_{N^{-1}}^{1-N^{-1}} \frac{t(1-t)}{\left\{\phi\left(\Phi^{-1}(t)\right)\right\}^{2}} d t=\log \log N+\log 2+\gamma+o(1) .
$$


With the aid of (5.56) we find

$$
\begin{aligned}
\pi_{N S}(\Phi, \theta)=1 & -\Phi\left(u_{\alpha}-\eta^{*}\right)+\frac{\eta^{*} \phi\left(u_{\alpha}-\eta^{*}\right)}{N}\left[-\frac{1}{2} \log \log N\right. \\
& \left.-\frac{1}{2} \log 2-\frac{1}{2} \gamma+\frac{1}{2}-\frac{1}{4}\left(u_{\alpha}^{2}-1\right)\right]+o\left(N^{-1}\right)
\end{aligned}
$$

where now $\eta^{*}=\{\lambda(1-\lambda) N\}^{\frac{1}{2}} \theta$, and

$$
\begin{aligned}
\pi_{N S}(\Lambda, \theta)=1 & -\Phi\left(u_{\alpha}-\bar{\eta}\right)+\frac{\bar{\eta} \phi\left(u_{\alpha}-\bar{\eta}\right)}{N}\left[\frac{1}{2} \log \log N+\frac{1}{2} \log 2+\frac{1}{2} \gamma\right. \\
& -\frac{3}{2}-\frac{1-3 \lambda+3 \lambda^{2}}{12 \lambda(1-\lambda)}\left(u_{\alpha}^{2}-1\right) \\
& +\left\{\frac{3 \frac{1}{2}(1-2 \lambda)^{2}}{4 \lambda(1-\lambda)}-\frac{\pi}{6}-\frac{4-21 \lambda+21 \lambda^{2}}{12 \lambda(1-\lambda)}\right\} u_{\alpha} \bar{\eta} \\
& +\left\{\frac{6\left(1-5 \lambda+5 \lambda^{2}\right)}{\lambda(1-\lambda)} \arctan 2^{\frac{1}{2}}-\frac{3 \frac{1}{2}(1-2 \lambda)^{2}}{4 \lambda(1-\lambda)}\right. \\
& \left.\left.-\frac{11 \pi\left(1-5 \lambda+5 \lambda^{2}\right)}{6 \lambda(1-\lambda)}+\frac{5-21 \lambda+21 \lambda^{2}}{12 \lambda(1-\lambda)}\right\} \bar{\eta}^{2}\right] \\
& +o\left(N^{-1}\right),
\end{aligned}
$$

where now $\bar{\eta}=\{\lambda(1-\lambda) N / \pi\}^{\frac{1}{2}} \theta$. Note that Theorem 5.2 ensures that expansion (5.57) is also valid for van der Waerden's two-sample test which is based on the approximate scores $a_{j}=\Phi^{-1}(j /(N+1))$.

It may be useful to remark here that an integral similar to (5.56) also occurs in $\mathrm{ABZ}$ (1976), formula (4.25) on page 130, where its asymptotic behavior is determined numerically. However, the numerically computed value is incorrect and in formulas (4.25) and (6.8) in $\mathrm{ABZ}(1976)$ the number $\frac{1}{2} \log 2+0.05832 \ldots$ should be replaced by $\frac{1}{2} \gamma=0.288608 \ldots$ (cf. the correction note in this issue).

6. The permutation test based on the sample means. In ABZ (1976) two results were given for permutation tests in the one-sample problem. The first of these is an asymptotic expansion for the power of the locally most powerful permutation test against contiguous shift alternatives. Secondly it was shown that the difference between the powers of the permutation test based on the sum of transformed observations $\sum j\left(X_{i}\right)$ and Student's test applied to $j\left(X_{1}\right), \cdots, j\left(X_{N}\right)$ is $o\left(N^{-1}\right)$ for a large class of alternatives.

In the present paper we shall forego the two-sample analogue of the first mentioned result; the expansion can be obtained in a straightforward manner in much the same way as in the one-sample case but the computations will be extremely tedious. We shall concentrate on the comparison with Student's test. For simplicity we take $j$ to be the identity, thus comparing the two-sample permutation test based on the sample means with Student's two-sample test. Also, we restrict attention to contiguous location alternatives. 
As before, we assume that $X_{1}, \cdots, X_{N}$ are independent, $X_{1}, \cdots, X_{m}$ having common df $F$ and $X_{m+1}, \cdots, X_{N}$ having common df $G(x)=F(x-\theta) ; Z=$ $\left(Z_{1}, \cdots, Z_{N}\right)$ denotes the vector of order statistics of $X_{1}, \cdots, X_{N}$. We wish to test the hypothesis $\theta=0$ against the alternative $\theta>0$ at a fixed level $\alpha \in(0,1)$. We denote probabilities and expectations under the alternative by $P$ and $E$, and under the hypothesis by $P_{F}$ and $E_{F}$. Note that we do not assume that $F$ has a density, as we did in the previous sections.

The permutation test rejects the hypothesis if

$$
\sum_{i=m+1}^{N} X_{i} \geqq \xi_{\alpha}(Z),
$$

possibly with randomization if equality occurs. Here $\xi_{\alpha}(Z)$ is chosen in such a way that

$$
P_{F}\left(\sum_{\imath=m+1}^{N} X_{i} \geqq \xi_{\alpha} \mid Z\right)=\alpha \quad \text { a.s. }
$$

with an obvious modification if there is randomization. If $F$ is known, Student's test rejects the hypothesis if

$$
\widetilde{T}=\frac{\{\lambda(1-\lambda) N(N-2)\}^{\frac{1}{2}}\left(X_{\bullet}{ }^{(2)}-X_{\bullet}{ }^{(1)}\right)}{\left[\sum_{i=1}^{m}\left(X_{i}-X_{\bullet}{ }^{(1)}\right)^{2}+\sum_{i=m+1}^{N}\left(X_{i}-X_{\bullet}{ }^{(2)}\right)^{2}\right]^{\frac{1}{2}}} \geqq t_{\alpha},
$$

where

$$
X_{\bullet}^{(1)}=\frac{1}{m} \sum_{i=1}^{m} X_{i}, \quad X_{\bullet}^{(2)}=\frac{1}{n} \sum_{i=m+1}^{N} X_{i}
$$

Here $t_{\alpha}$ depends on $F, N, \lambda$ and $\alpha$ and is chosen in such a way that the test has level $\alpha$. Again there may be randomization. Let $\pi_{P e}(F, \theta)$ and $\pi_{S t}(F, \theta)$ denote the power against the alternative $(F, F(\cdot-\theta))$ of the tests $(6.1)$ and $(6.3)$ respectively.

Theorem 6.1. Suppose that positive numbers $c, C, D, \varepsilon, \varepsilon^{\prime}, \delta$ and $r>8$ exist such that $F^{-1}$ is differentiable on an interval of length at least $\delta$ where

$$
\frac{d}{d t} F^{-1}(t) \geqq c,
$$

and such that $\int|x|^{r} d F(x) \leqq C, 0 \leqq \theta \leqq D N^{-\frac{1}{2}}, \varepsilon \leqq \lambda \leqq 1-\varepsilon$ and $\varepsilon^{\prime} \leqq \alpha \leqq 1-\varepsilon^{\prime}$. Then there exist $B>0$ depending only on $c, C, D, \varepsilon, \varepsilon^{\prime}$ and $\delta$, and $\beta>0$ depending only on $r$ such that

$$
\left|\pi_{P e}(F, \theta)-\pi_{S t}(F, \theta)\right| \leqq B N^{-1-\beta} .
$$

Proof. We shall draw heavily on the proof of Theorem 5.2 in ABZ (1976). The only essentially new problem is caused again by the occurrence of a term of order $N^{-\frac{1}{2}}$ in the expansions. The $O$ symbols in this proof are uniform for fixed $c, C, D, \varepsilon, \varepsilon^{\prime}$ and $\delta$. Since both tests are location invariant we may assume without loss of generality that $j x d F(x)=0$. 
We begin by collecting some results on moments that will be needed throughout the proof. Define

$$
\begin{gathered}
\beta=\min \left(\frac{r-8}{2 r+8}, \frac{1}{4}\right), \\
X_{\bullet}=Z_{\bullet}=\frac{1}{N} \sum_{i=1}^{N} X_{i}=\frac{1}{N} \sum_{j=1}^{N} Z_{j},
\end{gathered}
$$

and note that $E X_{N}{ }^{k}=E\left(X_{1}+\theta\right)^{k}$ and that $E X_{1}=0$. Proceeding as in the proof of Theorem 5.2 in $\mathrm{ABZ}$ (1976) we see that

$$
\frac{c^{2} \delta^{3}}{12} \leqq E X_{1}^{2} \leqq\left[E X_{1}^{4}\right]^{\frac{1}{2}} \leqq C^{2 / r},
$$

and that, uniformly on a set of probability $1-O\left(N^{-1-\beta}\right)$ under $P$ as well as under $P_{F}$,

$$
\begin{aligned}
\frac{1}{N} \sum_{i=1}^{m} X_{i}^{k}=(1-\lambda) E X_{1}{ }^{k}+O\left(N^{-\beta}\right), & & k=1, \ldots, 4, \\
\frac{1}{N} \sum_{i=m+1}^{N} X_{i}^{k}=\lambda E X_{1}{ }^{k}+O\left(N^{-\beta}\right), & k & =1, \ldots, 4, \\
\frac{1}{N} \sum_{i=1}^{m}\left(X_{i}-X_{\bullet}\right)^{k}=(1-\lambda) E X_{1}^{k}+O\left(N^{-\beta}\right), & k & =2, \ldots, 4, \\
\frac{1}{N} \sum_{i=m+1}^{N}\left(X_{i}-X_{\bullet}\right)^{k}=\lambda E X_{1}{ }^{k}+O\left(N^{-\beta}\right), & k & =2, \ldots, 4 .
\end{aligned}
$$

For $k=1,(6.9)$ and (6.10) are insufficient for our purposes. Arguing as in (5.13) in $\mathrm{ABZ}$ (1976) for $\tau=N^{-\frac{8}{8}}$, we find

$$
\frac{1}{N} \sum_{i=1}^{m} X_{i}=O\left(N^{-\frac{8}{8}}\right), \quad \frac{1}{N} \sum_{i=m+1}^{N} X_{i}=O\left(N^{-\frac{8}{8}}\right)
$$

uniformly with probability $1-O\left(N^{-1-\beta}\right)$ under both $P$ and $P_{F}$.

We shall also have to consider the quantity $l\left\{x: \exists i\left|x-X_{i}\right|<\zeta\right\}$ for some $\zeta \geqq N^{-\frac{3}{2}} \log N$, where $l$ denotes Lebesgue measure. Borrowing from the proof of Theorem 5.2 in $\mathrm{ABZ}$ (1976) again, we find that for $\zeta=N^{-\frac{3}{2}} \log N$,

$$
l\left\{x: \exists i \quad\left|x-X_{i}\right|<\zeta\right\} \geqq \frac{\delta N \zeta}{6}
$$

with probability $1-O\left(N^{-1-\beta}\right)$ both under $P$ and under $P_{F}$. Let $E_{1}$ be a set on which (6.9)-(6.14) hold uniformly, with $P\left(E_{1}\right)=1-O\left(N^{-1-\beta}\right)$ and $P_{F}\left(E_{1}\right)=$ $1-O\left(N^{-1-\beta}\right)$.

Under the hypothesis $P_{F}$ and conditional on $Z$ the df of

$$
N^{-\frac{1}{2}}\left(\sum_{i=m+1}^{N} X_{i}-\lambda \sum_{j=1}^{v} Z_{j}\right)
$$

equals $R(x, p)$ defined in (2.9) with $p_{j}=\lambda$ and $a_{j}=Z_{j}$ for $j=1, \cdots, N$. Hence Corollary 2.1 provides an expansion for this conditional df that holds 
uniformly on any set where the $a_{j}=Z_{j}$ satisfy (2.61) and (2.62) for some fixed positive $c, C$ and $\delta$, and in view of (6.8)-(6.14) such a set is contained in $E_{1}$. Since $\varepsilon^{\prime} \leqq \alpha \leqq 1-\varepsilon^{\prime}$, this yields an expansion for $\xi_{\alpha}(Z)$. We find (cf. (4.14))

$$
\begin{aligned}
& \frac{\xi_{\alpha}(Z)-\lambda \sum Z_{j}}{\left[\lambda(1-\lambda) \sum\left(Z_{j}-Z_{\bullet}\right)^{2}\right]^{\frac{1}{2}}} \\
& =u_{\alpha}+\frac{(1-2 \lambda) \sum\left(Z_{j}-Z_{\bullet}\right)^{3}}{6\{\lambda(1-\lambda)\}^{\frac{1}{2}}\left[\sum\left(Z_{j}-Z_{\bullet}\right)^{2}\right]^{\frac{3}{2}}}\left(u_{\alpha}{ }^{2}-1\right) \\
& \quad-\frac{(1-2 \lambda)^{2}\left[\sum\left(Z_{j}-Z_{\bullet}\right)^{3}\right]^{2}}{36 \lambda(1-\lambda)\left[\sum\left(Z_{j}-Z_{\bullet}\right)^{2}\right]^{3}}\left(2 u_{\alpha}{ }^{3}-5 u_{\alpha}\right)+\frac{u_{\alpha}}{2 N} \\
& \quad+\left\{\frac{\left(1-6 \lambda+6 \lambda^{2}\right) \sum\left(Z_{j}-Z_{\bullet}\right)^{4}}{24 \lambda(1-\lambda)\left[\sum\left(Z_{j}-Z_{\bullet}\right)^{2}\right.}-\frac{(1-2 \lambda)^{2}}{8 \lambda(1-\lambda) N}\right\}\left(u_{\alpha}{ }^{3}-3 u_{\alpha}\right) \\
& \quad+O\left(N^{-\frac{5}{4}}\right),
\end{aligned}
$$

uniformly on $E_{1}$.

Next we start to compute under the alternative $P$. We have $P\left(E_{1}\right)=1-$ $O\left(N^{-1-\beta}\right)$ and on $E_{1}$ we can use (6.8), (6.11) and (6.12) to replace the random terms of order $O\left(N^{-1}\right)$ on the right in $(6.15)$ by constants. In this way we arrive at

$$
\pi_{P e}(F, \theta)=P\left(T^{*} \geqq \xi_{\alpha}^{*}+U_{1}+O\left(N^{-1-\beta}\right)\right)+O\left(N^{-1-\beta}\right),
$$

where the first remainder term depends on $Z$ but may now be taken to be uniformly $O\left(N^{-1-\beta}\right)$, and where

$$
\begin{gathered}
T^{*}=\frac{\sum_{i=m+1}^{N} X_{i}-\lambda \sum Z_{j}}{\left[\lambda(1-\lambda) \sum\left(Z_{j}-Z_{\bullet}\right)^{2}\right]^{\frac{1}{2}}} \\
\xi_{\alpha}^{*}=u_{\alpha}+\frac{(1-2 \lambda) E X_{1}^{3}}{6\{\lambda(1-\lambda) N\}^{\frac{1}{2}}\left(E X_{1}^{2}\right)^{\frac{3}{2}}}\left(u_{\alpha}{ }^{2}-1\right) \\
-\frac{(1-2 \lambda)^{2}\left(E X_{1}^{3}\right)^{2}}{36 \lambda(1-\lambda) N\left(E X_{1}^{2}\right)^{3}}\left(2 u_{\alpha}^{3}-5 u_{\alpha}\right)+\frac{u_{\alpha}}{2 N} \\
+\left\{\frac{\left(1-6 \lambda+6 \lambda^{2}\right) E X_{1}^{4}}{24 \lambda(1-\lambda) N\left(E X_{1}^{2}\right)^{2}}-\frac{(1-2 \lambda)^{2}}{8 \lambda(1-\lambda) N}\right\}\left(u_{\alpha}{ }^{3}-3 u_{\alpha}\right), \\
U_{1}=\frac{(1-2 \lambda)\left(u_{\alpha}{ }^{2}-1\right)}{6\{\lambda(1-\lambda)\}^{\frac{1}{2}}}\left\{\frac{\sum\left(Z_{j}-Z\right)^{3}}{\left[\sum\left(Z_{j}-Z_{\bullet}\right)^{2}\right]^{\frac{3}{2}}}-\frac{N^{-\frac{1}{2}} E X_{1}^{3}}{\left(E X_{1}{ }^{3}\right)^{\frac{3}{2}}}\right\} .
\end{gathered}
$$

The basic problem is now to show that the rv $U_{1}$ originating from the $O\left(N^{-\frac{1}{2}}\right)$ term in (6.15), may be omitted in (6.16). Since $U_{1}$ is a rv of order $N^{-1}$, this problem is nontrivial. We shall show that because $U_{1}$ depends only on $Z$ and is approximately centered, a cancellation occurs which makes its contribution to (6.16) of negligible order. Several methods of proof are possible. We choose one that does not require any additional assumptions.

In (6.16), $P$ may be replaced by $P_{F}$ if $X_{i}$ is replaced by $X_{i}+\theta$ for $i=$ $m+1, \cdots, N$, which transforms $T^{*}$ and $U_{1}$ into $T^{*}(\theta)$ and $U_{1}(\theta)$, say. On the set $E_{1},(6.8)-(6.13)$ ensure that we can expand $T^{*}(\theta)$ and $U_{1}(\theta)$ about $T^{*}$ and 
$U_{1}$. Replacing rv's by their expected values if the difference is of negligible order, a simple calculation shows that under $P_{F}$ we have, uniformly on the set $E_{1}$,

$$
\begin{gathered}
T^{*}(\theta)=T^{*}\left[1-\frac{3 \lambda(1-\lambda) N \theta^{2}}{2 \sum\left(Z_{j}-Z_{\bullet}\right)^{2}}\right]-T^{* 2} \frac{\{\lambda(1-\lambda)\}^{\frac{1}{2}} \theta}{\left[\sum\left(Z_{j}-Z_{\bullet}\right)^{2}\right]^{\frac{1}{2}}} \\
+\frac{\{\lambda(1-\lambda)\}^{\frac{1}{2}} N \theta}{\left[\sum\left(Z_{j}-Z_{\bullet}\right)^{2}\right]^{\frac{1}{2}}}-\frac{\{\lambda(1-\lambda)\}^{\frac{3}{2}} N^{\frac{1}{2}} \theta^{3}}{2\left(E X_{1}^{2}\right)^{\frac{3}{2}}}+O\left(N^{-1-\beta}\right), \\
U_{1}(\theta)=U_{1}+O\left(N^{-1-\beta}\right) .
\end{gathered}
$$

Another easy calculation where we use (6.8)-(6.13) to bound the terms in (6.20) and (6.21) and to replace rv's by their expected values whenever possible, and where we note that $\xi_{\alpha}{ }^{*}=u_{\alpha}+O\left(N^{-\frac{1}{2}}\right)$, shows that uniformly on $E_{1}$, the inequality $T^{*}(\theta) \geqq \xi_{\alpha}{ }^{*}+U_{1}(\theta)+O\left(N^{-1-\beta}\right)$ is equivalent to $T^{*} \geqq \xi_{\alpha}{ }^{*}(\theta)-U_{0}+$ $U_{1}+O\left(N^{-1-\beta}\right)$, where

$$
\begin{gathered}
\xi_{\alpha} *(\theta)=\xi_{\alpha}{ }^{*}-\frac{\{\lambda(1-\lambda) N\}^{\frac{1}{2}} \theta}{\left(E X_{1}^{2}\right)^{\frac{1}{2}}}+\frac{\{\lambda(1-\lambda)\}^{\frac{1}{2}} \theta}{\left(N E X_{1}^{2}\right)^{\frac{1}{2}}} u_{\alpha}{ }^{2}-\frac{\lambda(1-\lambda) \theta^{2}}{2 E X_{1}^{2}} u_{\alpha}, \\
U_{0}=\{\lambda(1-\lambda)\}^{\frac{1}{2}} N \theta\left\{\frac{1}{\left[\sum\left(Z_{j}-Z_{\bullet}\right)^{2}\right]^{\frac{1}{2}}}-\frac{1}{\left(N E X_{1}^{2}\right)^{\frac{1}{2}}}\right\} .
\end{gathered}
$$

Since $P_{F}\left(E_{1}\right)=1-O\left(N^{-1-\beta}\right)$, this implies

$$
\begin{aligned}
\pi_{P e}(F, \theta) & =P_{F}\left(T^{*}(\theta) \geqq \xi_{\alpha}{ }^{*}+U_{1}(\theta)+O\left(N^{-1-\beta}\right)\right)+O\left(N^{-1-\beta}\right) \\
& =P_{F}\left(T^{*} \geqq \xi_{\alpha}{ }^{*}(\theta)-U_{0}+U_{1}+O\left(N^{-1-\beta}\right)\right)+O\left(N^{-1-\beta}\right),
\end{aligned}
$$

where the first remainder term in the last member depends on $X_{1}, \cdots, X_{N}$ but is uniformly $O\left(N^{-1-\beta}\right)$.

Since $U_{0}$ and $U_{1}$ depends on $X_{1}, \cdots, X_{N}$ only through $Z$, we can compute $P_{F}\left(T^{*} \leqq \xi_{\alpha}{ }^{*}(\theta)-U_{0}+U_{1}+O\left(N^{-1-\beta}\right)\right)$ by taking the expectation under $P_{F}$ of the conditional df of $T^{*}$ given $Z$ under $P_{F}$ evaluated at the point $\xi_{\alpha}{ }^{*}(\theta)-U_{0}+$ $U_{1}+O\left(N^{-1-\beta}\right)$. Corollary 2.1 provides an expansion for the conditional df of $T^{*}$ given $Z$ under $P_{F}$ that is valid uniformly on $E_{1}$, and $P_{F}\left(E_{1}\right)=1-O\left(N^{-1-\beta}\right)$. Combining these facts and simplifying as much as possible with the aid of (6.8)-(6.13) (note that (6.8), (6.11) and (6.12) imply that $U_{0}=O\left(N^{-\beta}\right)$ and $\left.U_{1}=O\left(N^{-\frac{1}{2}-\beta}\right)\right)$ we find

$$
\begin{aligned}
\pi_{P e}(F, \theta)=1 & -E_{F} \Phi\left(\xi_{\alpha}{ }^{*}(\theta)-U_{0}+U_{1}\right)+\frac{(1-2 \lambda)}{6\{\lambda(1-\lambda)\}^{\frac{1}{2}}} \\
& \times E_{F}\left[\frac{\sum\left(Z_{j}-Z_{\cdot}\right)^{3}}{\left[\sum\left(Z_{j}-Z_{\bullet}\right)^{2}\right]^{\frac{3}{2}}} \phi\left(\xi_{\alpha}{ }^{*}(\theta)-U_{0}\right) H_{2}\left(\xi_{\alpha} *(\theta)-U_{0}\right)\right] \\
& +\phi\left(\xi_{\alpha} *(\theta)\right)\left[\frac{1}{2 N} \xi_{\alpha}^{*}(\theta)+\left\{\frac{\left(1-6 \lambda+6 \lambda^{2}\right)}{24 \lambda(1-\lambda) N} \frac{E X_{1}^{4}}{\left(E X_{1}^{2}\right)^{2}}\right.\right. \\
& \left.-\frac{(1-2 \lambda)^{2}}{8 \lambda(1-\lambda) N}\right\} H_{3}\left(\xi_{\alpha}^{*}(\theta)\right)+\frac{(1-2 \lambda)^{2}}{72 \lambda(1-\lambda) N} \frac{\left(E X_{1}^{3}\right)^{2}}{\left(E X_{1}^{2}\right)^{3}} \\
& \left.\times H_{5}\left(\xi_{\alpha}{ }^{*}(\theta)\right)\right]+O\left(N^{-1-\beta}\right) .
\end{aligned}
$$


Thus we see that the contribution of $U_{1}$ to the expansion for $\pi_{P e}(F, \theta)$ is restricted to its contribution to $-E_{F} \Phi\left(\xi_{\alpha}{ }^{*}(\theta)-U_{0}+U_{1}\right)$. On the set $E_{1}$ we have $U_{1}=\tilde{U}_{1}+M$, where

$$
\tilde{U}_{1}=\frac{(1-2 \lambda)\left(u_{\alpha}{ }^{2}-1\right)}{6\{\lambda(1-\lambda) N\}^{\frac{1}{2}}}\left\{\left(\frac{\sum\left(Z_{j}-Z_{.}\right)^{3}}{N E X_{1}{ }^{3}}-1\right)-\frac{3}{2}\left(\frac{\sum\left(Z_{j}-Z_{\bullet}\right)^{2}}{N E X_{1}^{2}}-1\right)\right\},
$$

and

$N^{\frac{1}{2}} M=O\left(\left\{\frac{\sum\left(Z_{j}-Z_{\bullet}\right)^{2}}{N E X_{1}^{2}}-1\right\}^{2}+\left|\frac{\sum\left(Z_{j}-Z_{\bullet}\right)^{2}}{N E X_{1}^{2}}-1\right| \cdot\left|\frac{\sum\left(Z_{j}-Z_{\bullet}\right)^{3}}{N E X_{1}^{3}}-1\right|\right)$

uniformly on $E_{1}$; also $U_{0}=O\left(N^{-\beta}\right)$ uniformly on $E_{1}$. Let $\chi_{E_{1}}$ denote the indicator of $E_{1}$. Then, because $P_{F}\left(E_{1}\right)=1-O\left(N^{-1-\beta}\right)$,

$$
\begin{aligned}
E_{F} \Phi\left(\xi_{\alpha}{ }^{*}(\theta)-U_{0}+U_{1}\right)= & E_{F} \Phi\left(\xi_{\alpha}{ }^{*}(\theta)-U_{0} \chi_{E_{1}}+\tilde{U}_{1}+M \chi_{E_{1}}\right)+O\left(N^{-1-\beta}\right) \\
= & E_{F} \Phi\left(\xi_{\alpha}{ }^{*}(\theta)-U_{0} \chi_{E_{1}}\right)+E_{F} \phi\left(\xi_{\alpha}{ }^{*}(\theta)-U_{0} \chi_{E_{1}}\right) \tilde{U}_{1} \\
& \quad+O\left(N^{-1-\beta}+E_{F}\left\{\tilde{U}_{1}^{2}+|M| \chi_{E}\right\}\right) \\
= & E_{F} \Phi\left(\xi_{\alpha}{ }^{*}(\theta)-U_{0}\right)+\phi\left(\xi_{\alpha}{ }^{*}(\theta)\right) E_{F} \tilde{U}_{1} \\
& \quad+O\left(N^{-1-\beta}+E_{F}\left\{N^{-\beta}\left|\tilde{U}_{1}\right|+\tilde{U}_{1}^{2}+|M| \chi_{E}\right\}\right) .
\end{aligned}
$$

Noting that $\sum\left(Z_{j}-Z_{\bullet}\right)^{k}=\sum\left(X_{i}-X_{\bullet}\right)^{k}, E_{F} X_{i}=0$ and $E_{F}\left|X_{i}\right|^{r} \leqq C$ for some $r>8$, one easily verifies that $E_{F} \tilde{U}_{1}=O\left(N^{-\frac{3}{2}}\right), E_{F} \tilde{U}_{1}^{2}=O\left(N^{-2}\right)$ and $E_{F}|M| \chi_{E}=$ $O\left(N^{-\frac{3}{2}}\right)$. It follows that

$$
E_{F} \Phi\left(\xi_{\alpha}^{*}(\theta)-U_{0}+U_{1}\right)=E_{F} \Phi\left(\xi_{\alpha}^{*}(\theta)-U_{0}\right)+O\left(N^{-1-\beta}\right)
$$

and hence $U_{1}$ may be omitted in (6.25) because its contribution is of negligible order. Retracing our steps back to $(6.16)$ we conclude that the same must be true there, so that

$$
\pi_{P e}(F, \theta)=P\left(T^{*} \geqq \xi_{\alpha}^{*}+O\left(N^{-1-\beta}\right)\right)+O\left(N^{-1-\beta}\right) .
$$

The remainder of the proof parallels that of Theorem 5.2 in ABZ (1976). Let $\widetilde{T}$ be Student's statistic as defined in (6.3). The inequality $T^{*} \geqq a$ is algebraically equivalent to $\widetilde{T} \geqq a\left\{(N-2) /\left(N-a^{2}\right)\right\}^{\frac{1}{2}}$ on the set where $\sum\left(X_{i}-X_{0}\right)^{2} \neq 0$ and provided that $a^{2}<N$. Since $\sum\left(X_{i}-X_{\bullet}\right)^{2} \neq 0$ on $E_{1}$ for sufficiently large $N$ and $\varepsilon^{\prime} \leqq \alpha \leqq 1-\varepsilon^{\prime}$, this implies that

$$
\pi_{P e}(F, \theta)=P\left(\tilde{T} \geqq \xi_{\alpha}^{*}+\frac{u_{\alpha}^{3}-2 u_{\alpha}}{2 N}+O\left(N^{-1-\beta}\right)\right)+O\left(N^{-1-\beta}\right) .
$$

In the same way as in the proof of Theorem 5.2 in ABZ (1976) we show that

$$
\sup _{t} P\left(t \leqq \widetilde{T} \leqq t+O\left(N^{-1-\beta}\right)\right)=O\left(N^{-1-\beta}\right)
$$

and hence

$$
\pi_{P e}(F, \theta)=P\left(\widetilde{T} \geqq \xi_{\alpha}{ }^{*}+\frac{u_{\alpha}{ }^{3}-2 u_{\alpha}}{2 N}\right)+O\left(N^{-1-\beta}\right) .
$$

Now $\xi_{\alpha}{ }^{*}$ depends only on $N, \lambda, \alpha$ and $F$ but not on $\theta$, and arguing as in the 
proof of Theorem 5.2 in $\mathrm{ABZ}(1976)$ we find that this together with $\pi_{P e}(F, 0)=\alpha$ ensures that

$$
t_{\alpha}=\xi_{\alpha}^{*}+\frac{u_{\alpha}^{3}-2 u_{\alpha}}{2 N}+O\left(N^{-1-\beta}\right),
$$

with $t_{\alpha}$ defined as in (6.3). Combination of (6.29), (6.30) and (6.31) completes the proof.

Although we have conducted the proof in such a way as to avoid actually establishing expansions for $\pi_{P e}(F, \theta)$ and $\pi_{S t}(F, \theta)$, the excursion from (6.16) to (6.26) and back has, in fact, brought us rather close to obtaining such expansions. Suppose that the conditions of Theorem 6.1 are satisfied but drop the assumption $\int x d F(x)=0$ that was made in the proof merely for convenience. Define

$$
\begin{gathered}
\hat{\eta}=\frac{\{\lambda(1-\lambda) N\}^{\frac{1}{2}} \theta}{\sigma\left(X_{1}\right)}, \\
\kappa_{3}(F)=\frac{E\left(X_{1}-E X_{1}\right)^{3}}{\sigma^{3}\left(X_{1}\right)}, \quad \kappa_{4}(F)=\frac{E\left(X_{1}-E X_{1}\right)^{4}}{\sigma^{4}\left(X_{1}\right)}-3,
\end{gathered}
$$

where all moments are computed under $F$ since only $X_{1}$ is involved. A relatively straightforward computation starting with (6.25) and (6.26) yields

$$
\begin{aligned}
\pi_{P e}(F, \theta)=1 & -\Phi\left(u_{\alpha}-\hat{\eta}\right)+\frac{\hat{\eta} \phi\left(u_{\alpha}-\hat{\eta}\right)}{72}\left[\frac{12(1-2 \lambda) \kappa_{3}(F)}{\{\lambda(1-\lambda) N\}^{\frac{1}{2}}}\left(\hat{\eta}-2 u_{\alpha}\right)\right. \\
& +\frac{(1-2 \lambda)^{2} \kappa_{3}{ }^{2}(F)}{\lambda(1-\lambda) N}\left(-\hat{\eta}^{4}+5 u_{\alpha} \hat{\eta}^{3}-8 u_{\alpha}{ }^{2} \hat{\eta}^{2}+4 u_{\alpha}{ }^{3} \hat{\eta}+8 \hat{\eta}^{2}\right. \\
& \left.-24 u_{\alpha} \hat{\eta}+20 u_{\alpha}{ }^{2}-10\right) \\
& +\frac{3 \kappa_{4}(F)}{\lambda(1-\lambda) N}\left\{-\left(1-3 \lambda+3 \lambda^{2}\right)\left(\hat{\eta}^{2}-3\right)\right. \\
& \left.+3\left(1-5 \lambda+5 \lambda^{2}\right) u_{\alpha} \hat{\eta}-3\left(1-6 \lambda+6 \lambda^{2}\right) u_{\alpha}{ }^{2}\right\} \\
& \left.-\frac{18 u_{\alpha}{ }^{2}}{N}\right]+O\left(N^{-1-\beta}\right),
\end{aligned}
$$

where $\beta$ is given by (6.6) and the $O$ symbol is uniform for fixed $c, C, D, \varepsilon, \varepsilon^{\prime}$ and $\delta$. Theorem 6.1 ensures that the same expansion is valid for $\pi_{S t}(F, \theta)$.

The case where $F$ is normal is perhaps of most interest because both tests are then asymptotically efficient. Since $\Phi$ satisfies the stronger regularity conditions needed to replace $\beta$ by $\frac{1}{2}$ we find in this case

$$
\begin{aligned}
\pi_{P e}(\Phi, \theta) & =\pi_{S t}(\Phi, \theta)+O\left(N^{-\frac{3}{2}}\right) \\
& =1-\Phi\left(u_{\alpha}-\eta^{*}\right)-\frac{u_{\alpha}{ }^{2} \eta^{*} \phi\left(u_{\alpha}-\eta^{*}\right)}{4 N}+O\left(N^{-\frac{3}{2}}\right),
\end{aligned}
$$

where $\eta^{*}=\{\lambda(1-\lambda) N\}^{\frac{1}{2}} \theta$.

7. Deficiencies of distributionfree tests. In analogy to the one-sample case we want to compare the distributionfree tests discussed so far to the best parametric tests for the two-sample problem when the hypothesis and the alternative are 
both simple. The situation is more complicated than in the one-sample case because of the shift invariance of the distributionfree tests involved. Let $X_{1}, \cdots, X_{N}$ be independent and let $(F, G)$ denote the hypothesis that $X_{1}, \cdots, X_{m}$ have common df $F$ and $X_{m+1}, \cdots, X_{N}$ have common df $G$. For fixed $F$ and $\theta$ and varying $\Delta \in R^{1}$, consider the simple hypothesis $H_{F}$ and the simple alternative $K_{F, \theta, \Delta}$, where

$$
H_{F}=(F, F), \quad K_{F, \theta, \Delta}=(F(\cdot+\Delta \theta), F(\cdot-(1-\Delta) \theta)) .
$$

The shift invariance of the distributionfree tests ensures that their power against $K_{F, \theta, \Delta}$ is independent of $\Delta$, so that it was sufficient to consider only alternatives with $\Delta=0$ in the preceding sections. Note that the form of the locally most powerful rank test against $K_{F, \theta, \Delta}$ is also independent of $\Delta$. However, the envelope power $\pi^{+}(F, \theta, \Delta)$, i.e., the power of the most powerful level $\alpha$ test of $H_{F}$ against $K_{F, \theta, \Delta}$, does depend on $\Delta$ and the "right" $\Delta$ against which comparisons should be made is thus the value $\Delta_{0}$ that minimizes the envelope power. It is given to first order by $\Delta_{0} \sim \lambda$. For values of $\Delta$ whose asymptote is different there is not even an asymptotically efficient shift invariant test, so that the deficiency of a shift invariant test with respect to the best test is not of much interest in this case. Of course we shall have to provide a more precise asymptotic evaluation of $\Delta_{0}$ because we are concerned with second order terms.

Suppose that $F$ is a fixed df with density $f$ that is positive and five times differentiable on $R^{1}$. The most powerful level $\alpha$ test for $H_{F}$ against $K_{F, \theta, \Delta}$ rejects $H_{F}$ for large values of the statistic

$$
S_{\theta, \Delta}=\sum_{i=1}^{m} \log \frac{f\left(X_{i}+\Delta \theta\right)}{f\left(X_{i}\right)}+\sum_{i=m+1}^{N} \log \frac{f\left(X_{i}-(1-\Delta) \theta\right)}{f\left(X_{i}\right)} .
$$

This statistic is a sum of independent rv's and we can therefore obtain an Edgeworth expansion for its df under $H_{F}$ and under $K_{F, \theta, \Delta}$ and hence for the power $\pi^{+}(F, \theta, \Delta)$ by proceeding in the classical manner and expanding the cumulants of the statistic. In this expansion for $\pi^{+}(F, \theta, \Delta)$ we minimize with respect to $\Delta$. We shall give each of these expansions but we omit the tedicus computations.

Define $\Psi_{i}$ by (4.15) for $i=1, \cdots, 5$, and take

$$
\begin{aligned}
\tilde{\pi}^{+}(F, \theta, \Delta) & \\
=1 & -\Phi\left(u_{\alpha}-\tilde{\eta}\right)-\frac{\tilde{\eta} \phi\left(u_{\alpha}-\tilde{\eta}\right)}{288}\left[\frac{24}{N^{\frac{1}{2}}} \frac{\tau_{3} \int \Psi_{1}{ }^{3}(t) d t}{\left\{\tau_{2} \int \Psi_{1}{ }^{2}(t) d t\right\}^{\frac{3}{2}}}\left(-2 u_{\alpha}+\tilde{\eta}\right)\right. \\
& +\frac{4}{N} \frac{\tau_{4} \int \Psi_{1}^{4}(t) d t}{\left\{\tau_{2} \int \Psi_{1}^{2}(t) d t\right\}^{2}}\left\{-3\left(u_{\alpha}{ }^{2}-1\right)+3 \tilde{\eta} u_{\alpha}-2 \tilde{\eta}^{2}\right\} \\
& +\frac{12}{N} \frac{\tau_{4} \int \Psi_{2}{ }^{2}(t) d t}{\left\{\tau_{2} \int \Psi_{1}^{2}(t) d t\right\}^{2}} \bar{\eta}^{2}+\frac{1}{N} \frac{\left\{\tau_{3} \int \Psi_{1}^{3}(t) d t\right\}^{2}}{\left\{\tau_{2} \int \Psi_{1}^{2}(t) d t\right\}^{3}} \\
& \times\left\{8\left(2 u_{\alpha}{ }^{2}-1\right)-4 \tilde{\eta}\left(u_{\alpha}{ }^{3}+3 u_{\alpha}\right)+\tilde{\eta}^{2}\left(8 u_{\alpha}{ }^{2}+1\right)-5 \tilde{\eta}^{3} u_{\alpha}+\tilde{\eta}^{4}\right\} \\
& \left.-\frac{36}{N} \frac{\tau_{4}}{\tau_{2}{ }^{2}}\left(-u_{\alpha}{ }^{2}+1+\tilde{\eta}_{\alpha}\right)\right],
\end{aligned}
$$


where

$$
\begin{array}{cl}
\tilde{\eta}=\left[N\left\{(1-\lambda) \Delta^{2}+\lambda(1-\Delta)^{2}\right\} \int \Psi_{1}^{2}(t) d t\right]^{\frac{1}{2}} \theta, & \\
\tau_{k}=(1-\lambda) \Delta^{k}+\lambda(\Delta-1)^{k}, & k=2,3,4 .
\end{array}
$$

LemMa 7.1. Let $F$ satisfy (5.6) for $m_{i}=5 / i, i=1, \ldots, 5$, and suppose that positive numbers $D, D^{\prime}, \varepsilon$ and $\varepsilon^{\prime}$ exist such that $0 \leqq \theta \leqq D N^{-\frac{1}{2}},|\Delta \theta| \leqq D^{\prime} N^{-\frac{1}{2}}$, $\varepsilon \leqq \lambda \leqq 1-\varepsilon$ and $\varepsilon^{\prime} \leqq \alpha \leqq 1-\varepsilon^{\prime}$. Then there exists $B>0$ depending only on $F, D, D^{\prime}, \varepsilon$ and $\varepsilon^{\prime}$ such that

$$
\left|\pi^{+}(F, \theta, \Delta)-\tilde{\pi}^{+}(F, \theta, \Delta)\right| \leqq B N^{-\frac{3}{2}} .
$$

Proof. Under the conditions of the lemma we find that under $H_{F}=(F, F)$

$$
\begin{aligned}
P_{F}\left(\frac{S_{\theta, \Delta}+\frac{1}{2} \tilde{\eta}^{2}}{\tilde{\eta}} \leqq x\right) \\
=\Phi(x)-\frac{\phi(x)}{288}\left[\frac{24}{N^{\frac{1}{2}}} \frac{\tau_{3} \int \Psi_{1}^{3}(t) d t}{\left\{\tau_{2} \int \Psi_{1}^{2}(t) d t\right\}^{\frac{3}{2}}}\left\{2\left(x^{2}-1\right)-3 \tilde{\eta} x+\tilde{\eta}^{2}\right\}\right. \\
\quad+\frac{4}{N} \frac{\tau_{4} \int \Psi_{1}^{4}(t) d t}{\left\{\tau_{2} \int \Psi_{1}^{2}(t) d t\right\}^{2}}\left\{3\left(x^{3}-3 x\right)-6 \tilde{\eta}\left(x^{2}-1\right)+5 \tilde{\eta}^{2} x-2 \tilde{\eta}^{3}\right\} \\
+\frac{12}{N} \frac{\tau_{4} \int \Psi_{2}^{2}(t) d t}{\left\{\tau_{2} \int \Psi_{1}^{2}(t) d t\right\}^{2}}\left\{-\tilde{\eta}^{2} x+\tilde{\eta}^{3}\right\} \\
+\frac{1}{N} \frac{\left\{\tau_{3} \int \Psi_{1}^{3}(t) d t\right\}^{2}}{\left\{\tau_{2} \int \Psi_{1}^{2}(t) d t\right\}^{3}}\left\{4\left(x^{5}-10 x^{3}+15 x\right)-12 \tilde{\eta}\left(x^{4}-6 x^{2}+3\right)\right. \\
\left.+13 \tilde{\eta}^{2}\left(x^{3}-3 x\right)-6 \tilde{\eta}^{3}\left(x^{2}-1\right)+\tilde{\eta}^{4} x\right\} \\
\left.\quad+\frac{36 \tau_{4}}{N \tau_{2}^{2}}\left\{-\left(x^{3}-3 x\right)+2 \tilde{\eta}\left(x^{2}-1\right)-\tilde{\eta}^{2} x\right\}\right]+O\left(N^{-\frac{3}{2}}\right)
\end{aligned}
$$

whereas under $K_{F, \theta, \Delta}$,

$$
\begin{aligned}
P\left(\frac{S_{\theta, \Delta}-\frac{1}{2} \tilde{\eta}^{2}}{\tilde{\eta}} \leqq x\right) \\
=\Phi(x)-\frac{\phi(x)}{288}\left[\frac{24}{N^{\frac{1}{2}}} \frac{\tau_{3} \int \Psi_{1}^{3}(t) d t}{\left\{\tau_{2} \int \Psi_{1}^{2}(t) d t\right\}^{\frac{3}{2}}}\left\{2\left(x^{2}-1\right)+3 \tilde{\eta} x+\tilde{\eta}^{2}\right\}\right. \\
+\frac{4}{N} \frac{\tau_{4} \int \Psi_{1}^{4}(t) d t}{\left\{\tau_{2} \int \Psi_{1}^{2}(t) d t\right\}^{2}}\left\{3\left(x^{3}-3 x\right)+6 \tilde{\eta}\left(x^{2}-1\right)+5 \tilde{\eta}^{2} x+2 \tilde{\eta}^{3}\right\} \\
+\frac{12}{N} \frac{\tau_{4} \int \Psi_{2}^{2}(t) d t}{\left\{\tau_{2} \int \Psi_{1}^{2}(t) d t\right\}^{2}}\left\{-\tilde{\eta}^{2} x-\tilde{\eta}^{3}\right\} \\
+\frac{1}{N} \frac{\left\{\tau_{3} \int \Psi_{1}^{3}(t) d t\right\}^{2}}{\left\{\tau_{2} \int \Psi_{1}^{2}(t) d t\right\}^{3}}\left\{4\left(x^{5}-10 x^{3}+15 x\right)+12 \tilde{\eta}\left(x^{4}-6 x^{2}+3\right)\right. \\
\left.+13 \tilde{\eta}^{2}\left(x^{3}-3 x\right)+6 \tilde{\eta}^{3}\left(x^{2}-1\right)+\tilde{\eta}^{4} x\right\} \\
\left.+\frac{36 \tau_{4}}{N \tau_{2}^{2}}\left\{-\left(x^{3}-3 x\right)-2 \tilde{\eta}\left(x^{2}-1\right)-\tilde{\eta}^{2} x\right\}\right]+O\left(N^{-\frac{3}{2}}\right) .
\end{aligned}
$$

The remainder terms $O\left(N^{-\frac{3}{2}}\right)$ are uniform for fixed $F, D, D^{\prime} \varepsilon$ and $\varepsilon^{\prime}$. Together these expansions yield (7.4). 
For large values of $\left|\Delta \theta N^{\frac{1}{}}\right|$, both $\pi^{+}(F, \theta, \Delta)$ and $\tilde{\pi}^{+}(F, \theta, \Delta)$ will come close to 1 as $N \rightarrow \infty$. It follows that an asymptotic expansion for the value $\Delta_{0}$ that minimizes $\pi^{+}(F, \theta, \Delta)$ may be obtained by minimizing $\tilde{\pi}^{+}(F, \theta, \Delta)$ instead. This yields

$$
\Delta_{0}=\lambda+\frac{\{\lambda(1-\lambda)\}^{\frac{1}{2}}}{4 N^{\frac{1}{2}}} \frac{\int \Psi_{1}^{3}(t) d t}{\left\{\int \Psi_{1}^{2}(t) d t\right\}^{\frac{3}{2}}}\left(-2 u_{\alpha}+\eta^{*}\right)+O\left(N^{-1}\right)
$$

with $\eta^{*}$ as in (5.29). Since the derivative of $\tilde{\eta}$ with respect to $\Delta$ vanishes at $\Delta=\lambda,(7: 5)$ is sufficient to determine $\tilde{\eta}$ and $\tilde{\pi}^{+}$for $\Delta=\Delta_{0}$ up to a remainder $O\left(N^{-\frac{3}{2}}\right)$. Noting that indeed $\left|\Delta_{0} \theta\right|=O\left(N^{-\frac{1}{2}}\right)$, we substitute (7.5) for $\Delta$ in (7.1) (7.3) and neglecting terms that are $O\left(N^{-\frac{3}{2}}\right)$, we find that $\tilde{\pi}^{+}\left(F, \theta, \Delta_{0}\right)$ reduces to

$$
\begin{aligned}
\tilde{\pi}^{+}(F, \theta)=1 & -\Phi\left(u_{\alpha}-\eta^{*}\right)+\frac{\eta^{*} \phi\left(u_{\alpha}-\eta^{*}\right)}{288} \\
& \times\left[\frac{24(1-2 \lambda)}{\{\lambda(1-\lambda) N\}^{\frac{1}{2}}} \frac{\int \Psi_{1}{ }^{3}(t) d t}{\left\{\int \Psi_{1}{ }^{2}(t) d t\right\}^{\frac{3}{2}}}\left(-2 u_{\alpha}+\eta^{*}\right)\right. \\
& +\frac{4\left(1-3 \lambda+3 \lambda^{2}\right)}{\lambda(1-\lambda) N} \frac{\int \Psi_{1}{ }^{4}(t) d t}{\left\{\int \Psi_{1}{ }^{2}(t) d t\right\}^{2}} \\
& \times\left\{3\left(u_{\alpha}{ }^{2}-1\right)-3 \eta^{*} u_{\alpha}+2 \eta^{* 2}\right\} \\
& -\frac{12\left(1-3 \lambda+3 \lambda^{2}\right)}{\lambda(1-\lambda) N} \frac{\int \Psi_{2}{ }^{2}(t) d t}{\left\{\int \Psi_{1}{ }^{2}(t) d t\right\}^{2}} \eta^{* 2}-\frac{9}{N} \frac{\left\{\int \Psi_{1}{ }^{3}(t) d t\right\}^{2}}{\left\{\int \Psi_{1}^{2}(t) d t\right\}^{3}} \\
& \times\left(2 u_{\alpha}-\eta^{*}\right)^{2}+\frac{(1-2 \lambda)^{2}}{\lambda(1-\lambda)^{2} N} \frac{\left\{\int \Psi_{1}{ }^{2}(t) d t\right\}^{2}}{\left\{\int \Psi_{1}{ }^{2}(t) d t\right\}^{3}}\left\{-8\left(2 u_{\alpha}{ }^{2}-1\right)\right. \\
& \left.+4 \eta^{*}\left(u_{\alpha}{ }^{3}+3 u_{\alpha}\right)-\eta^{* 2}\left(8 u_{\alpha}{ }^{2}+1\right)+5 \eta^{*} u_{\alpha}-\eta^{* 4}\right\} \\
& \left.+\frac{36\left(1-3 \lambda+3 \lambda^{2}\right)}{\lambda(1-\lambda) N}\left\{-\left(u_{\alpha}{ }^{2}-1\right)+\eta^{*} u_{\alpha}\right\}\right]
\end{aligned}
$$

with $\eta^{*}$ as in (5.29). Summarizing, we have

Lemma 7.2. Let $F$ satisfy (5.6) for $m_{i}=5 / i, i=1, \ldots, 5$, and suppose that positive numbers $D, \varepsilon$ and $\varepsilon^{\prime}$ exist such that $0 \leqq \theta \leqq D N^{-\frac{1}{2}}, \varepsilon \leqq \lambda \leqq 1-\varepsilon$ and $\varepsilon^{\prime} \leqq \alpha \leqq 1-\varepsilon^{\prime}$. Then there exists $B>0$ depending only on $F, D, \varepsilon$ and $\varepsilon^{\prime}$ such that

$$
\left|\pi^{+}\left(F, \theta, \Delta_{0}\right)-\tilde{\pi}^{+}(F, \theta)\right| \leqq B N^{-\frac{3}{2}} .
$$

For the same testing problem Theorem 5.2 provides an expansion for the power $\pi(F, \theta)$ of the locally most powerful rank test. Together, Theorem 5.2 and Lemma 7.2 enable us to find an asymptotic expression for the deficiency $d_{N}$ of the locally most powerful rank test with respect to the most powerful parametric test for $H_{F}$ against $K_{F, \theta, \Delta_{0}}$. To ensure that $F$ satisfies the assumptions of both Theorem 5.2 and Lemma 7.2, we require that $F \in \mathscr{F}_{1}$, where

Definition 7.1. $\mathscr{F}_{1}$ is the class of df's $F$ on $R^{1}$ with positived and five times differentiable densities $f$ and such that (5.6) is satisfied for $i=1, \ldots, 5$ with $m_{1}=6, m_{2}=3, m_{3}=\frac{5}{3}, m_{4}=\frac{5}{4}, m_{5}=1$, and such that (5.7) holds. 
Furthermore, we define

$$
\begin{aligned}
\tilde{d}_{N, 0}=\frac{1}{48} & {\left[\frac{4 \int \Psi_{1}^{4}(t) d t}{\left\{\int \Psi_{1}^{2}(t) d t\right\}^{2}}\left\{3\left(u_{\alpha}^{2}-1\right)-2 \eta^{*} u_{\alpha}\right\}\right.} \\
& -\frac{4\left(1-3 \lambda+3 \lambda^{2}\right)}{\lambda(1-\lambda)}\left\{\frac{\int \Psi_{1}^{4}(t) d t-3 \int \Psi_{2}^{2}(t) d t}{\left\{\int \Psi_{1}^{2}(t) d t\right\}^{2}}+3\right\} \eta^{* 2} \\
& -\frac{3\left\{\int \Psi_{1}{ }^{3}(t) d t\right\}^{2}}{\left\{\int \Psi_{1}^{2}(t) d t\right\}^{3}}\left(2 u_{\alpha}-\eta^{*}\right)^{2}-\frac{3(1-2 \lambda)^{2}}{\lambda(1-\lambda)} \frac{\left\{\int \Psi_{1}{ }^{3}(t) d t\right\}^{2}}{\left\{\int \Psi_{1}^{2}(t) d t\right\}^{3}} \eta^{* 2} \\
& \left.-12\left\{u_{\alpha}^{2}+3-2 \eta^{*} u_{\alpha}\right\}\right] \\
\tilde{d}_{N, 1}=\tilde{d}_{N, 0} & +\frac{1}{\int \Psi_{1}^{2}(t) d t} \sum_{j=1}^{N} \sigma^{2}\left(\Psi_{1}\left(U_{j: N}\right)\right) \\
\tilde{d}_{N, 2}=\tilde{d}_{N, 0} & +\frac{1}{\int \Psi_{1}^{2}(t) d t} \int_{N^{-1}}^{1-N-1}\left(\Psi_{1}^{\prime}(t)\right)^{2} t(1-t) d t \\
\tilde{d}_{N, 3}= & \tilde{d}_{N, 0}+\frac{1}{\int \Psi_{1}^{2}(t) d t} \sum_{j=1}^{N} E\left(\Psi_{1}\left(U_{j: N}\right)-\Psi_{1}\left(\frac{j}{N+1}\right)\right)^{2}
\end{aligned}
$$

where $\Psi_{i}$ and $\eta^{*}$ are given by (4.15) and (5.29) and $U_{j: N}$ is the $j$ th order statistic of a sample of size $N$ from the uniform distribution on $(0,1)$.

THEOREM 7.1. Let $d_{N}$ be the deficiency of the locally most powerful rank test with respect to the most powerful test for testing $H_{F}$ against $K_{F, \theta, \Delta_{0}}$ on the basis of $X_{1}, \cdots, X_{N}$ and at level $\alpha$. Suppose that $F \in \mathscr{F}_{1}, c N^{-\frac{1}{2}} \leqq \theta \leqq C N^{-\frac{1}{2}}, \varepsilon \leqq \lambda \leqq$ $1-\varepsilon$ and $\varepsilon^{\prime} \leqq \alpha \leqq 1-\varepsilon^{\prime}$ for positive $c, C$, $\varepsilon$ and $\varepsilon^{\prime}$. Then, for every fixed $F, c$, $C, \varepsilon$ and $\varepsilon^{\prime}$, there exist positive numbers $B, \delta_{1}, \delta_{2}, \cdots$ with $\lim _{N \rightarrow \infty} \delta_{N}=0$ such that

$$
\begin{gathered}
\left|d_{N}-\tilde{d}_{N, 1}\right| \leqq \delta_{N} \\
\left|d_{N}-\tilde{d}_{N, 2}\right| \leqq \delta_{N}+B N^{-\frac{1}{2}} \int_{N^{-1}}^{1-1}\left(\Psi_{1}^{\prime}(t)\right)^{2}\{t(1-t)\}^{\frac{1}{2}} d t
\end{gathered}
$$

If in the above the locally most powerful rank test is replaced by the rank test with the corresponding approximate scores $a_{j}=-\Psi_{1}(j /(N+1))$ then

$$
\left|d_{N}-\tilde{d}_{N, 3}\right| \leqq \delta_{N}
$$

and (7.10) continues to hold.

Proof. Let us first consider the locally most powerful rank test and show that the expansions (5.35) and (7.7) yield (7.9). The conditions of the theorem ensure that $\eta^{*},\{\lambda(1-\lambda)\}^{-1}$ and $u_{\alpha}$ are bounded. As $\mathscr{F}_{1}^{-} \subset F^{*},(5.18)$ holds and the reasoning leading up to $(5.46)$ gives

$$
\begin{aligned}
N^{-1} \sum_{j=1}^{N} \sigma^{2}\left(\Psi_{1}\left(U_{j: N}\right)\right) & \leqq N^{-1} \sum_{j=1}^{N} E\left\{\Psi_{1}\left(U_{j: N}\right)-\Psi_{1}\left(\frac{j}{N+1}\right)\right\}^{2} \\
& =o\left(N^{-\frac{2}{3}}\right) .
\end{aligned}
$$

In view of these remarks we find from $(5.35),(5.32)$ and $(5.31)$ that the power 
$\pi(F, \theta)$ of the locally most powerful rank test satisfies

$$
\begin{aligned}
\pi(F, \theta)=1 & -\Phi\left(u_{\alpha}-\eta^{*}\right) \\
& +\frac{\eta^{*} \phi\left(u_{\alpha}-\eta^{*}\right)}{12} \frac{(1-2 \lambda)}{\{\lambda(1-\lambda) N\}^{\frac{1}{2}}} \frac{\int \Psi_{1}{ }^{3}(t) d t}{\left\{\int \Psi_{1}^{2}(t) d t\right\}^{\frac{3}{2}}}\left(-2 u_{\alpha}+\eta^{*}\right) \\
& +o\left(N^{-\frac{3}{3}}\right) .
\end{aligned}
$$

From Lemma 7.2 and (7.6) it is clear that $\pi^{+}\left(F, \theta, \Delta_{0}\right)$ also equals the right-hand side of (7.13). Since $d_{N}$ is obtained by replacing $N$ and $\eta^{*}$ by $\left(N+d_{N}\right)$ and $\eta^{*}\left(1+d_{N^{\prime}} N^{-1}\right)^{\frac{1}{2}}$ in $\pi(F, \theta)$ and equating the result to $\pi^{+}\left(F, \theta, \Delta_{0}\right)$, and since $\eta^{*}$ is bounded away from zero, we find that $d_{v}=o\left(N^{\frac{1}{3}}\right)$.

Having obtained this crude bound for $d_{N}$ we study the effect of the substitution of $\left(N+d_{v}\right)$ and $\eta^{*}\left(1+d_{v} N^{-1}\right)^{\frac{1}{2}}$ for $N$ and $\eta^{*}$ a bit more carefully. The effect on $\pi_{0}^{*}(F, \theta)$ as given in (5.31) is obviously the addition of a term

$$
\frac{\eta^{*} \phi\left(u_{\alpha}-\eta^{*}\right)}{2 N} d_{N}+o\left(N^{-\frac{7}{6}}\right)
$$

to prove that this remains true for $\pi_{1}^{*}(F, \theta)$ in $(5.32)$ it is clearly sufficient to show that

$$
\frac{1}{N} \sum_{j=1}^{i} \sigma^{2}\left(\Psi_{1}\left(U_{j: N}\right)\right)=\frac{1}{N+1} \sum_{j=1}^{Y+1} \sigma^{2}\left(\Psi_{1}\left(U_{j: N+1}\right)\right)+o\left(N^{-\frac{5}{3}}\right) .
$$

Once this has been established, (5.35) and (7.7) imply that an expansion for $d_{N}$ may be obtained by equating (7.14) to $\tilde{\pi}^{+}(F, \theta)-\pi_{1}^{*}(F, \theta)+o\left(N^{-1}\right)$ and an easy computation yields (7.9).

To prove (7.15), we let $b_{j, v}$ denote the density of $U_{j: N}$ and we note the wellknown recurrence relation $(N+1) b_{j, N}=j b_{j+1, N+1}+(N-j+1) b_{j, N+1}$. We have

$$
\begin{aligned}
\sigma^{2}\left(\Psi_{1}\left(U_{j: N}\right)\right)= & \frac{j}{N+1} E\left\{\Psi_{1}\left(U_{j+1: N+1}\right)-E \Psi_{1}\left(U_{j: N}\right)\right\}^{2} \\
& \quad+\frac{N-j+1}{N+1} E\left\{\Psi_{1}\left(U_{j: N+1}\right)-E \Psi_{1}\left(U_{j: N}\right)\right\}^{2} \\
= & \frac{j}{N+1} \sigma^{2}\left(\Psi_{1}\left(U_{j+1: N+1}\right)\right)+\frac{N-j+1}{N+1} \sigma^{2}\left(\Psi_{1}\left(U_{j: N+1}\right)\right) \\
& \quad+\frac{j(N-j+1)}{(N+1)^{2}}\left\{E\left[\Psi_{1}\left(U_{j+1: N+1}\right)-\Psi_{1}\left(U_{j: N+1}\right)\right]\right\}^{2} .
\end{aligned}
$$

Summation on $j$ gives

$$
\begin{aligned}
& \frac{1}{N} \sum_{j=1}^{N} \sigma^{2}\left(\Psi_{1}\left(U_{j: N}\right)\right) \\
& =\frac{1}{N+1} \sum_{j=1}^{v+1} \sigma^{2}\left(\Psi_{1}\left(U_{j: N+1}\right)\right) \\
& \quad+\sum_{j=1}^{N} \frac{j(N-j+1)}{N(N+1)^{2}}\left\{E\left[\Psi_{1}\left(U_{j+1: N+1}\right)-\Psi_{1}\left(U_{j: N+1}\right)\right]\right\}^{2}
\end{aligned}
$$


By Fubini's theorem and (5.18),

$$
\begin{aligned}
\left|E\left[\Psi_{1}\left(U_{j+1: N+1}\right)-\Psi_{1}\left(U_{j: N+1}\right)\right]\right| & \leqq E \int_{U_{j: N+1}^{j} j: N+1}^{U_{j: 1}}\left|\Psi_{1}{ }^{\prime}(t)\right| d t \\
& =\int_{0}^{1}\left|\Psi_{1}{ }^{\prime}(t)\right| P\left(U_{j: N+1} \leqq t<U_{j+1: N+1}\right) d t \\
& =\left(\begin{array}{c}
N+1 \\
j
\end{array}\right) \int_{0}^{1}\left|\Psi_{1}^{\prime}(t)\right| t^{j}(1-t)^{N+1-j} d t \\
& \leqq M\left(\frac{j}{N+1}\right) \frac{(N+1)^{\frac{4}{3}}}{\{j(N+1-j)\}^{\frac{7}{6}}},
\end{aligned}
$$

where $M$ is a bounded function on $(0,1)$ with $\lim _{t \rightarrow 0,1} M(t)=0$. Hence

$$
\begin{aligned}
\sum_{j=1}^{N} \frac{j(N-j+1)}{N(N+1)^{2}} & \left\{E\left[\Psi_{1}\left(U_{j+1: N+1}\right)-\Psi_{1}\left(U_{j: N+1}\right)\right]\right\}^{2} \\
= & o\left(N^{-2} \int_{N^{-1}}^{1-N-1}\{t(1-t)\}^{-\frac{4}{3}} d t\right)=o\left(N^{-\frac{5}{3}}\right) .
\end{aligned}
$$

Together with (7.16), this proves (7.15) and establishes expansion (7.9).

For the rank test based on the approximate scores the proof that (5.39) and (7.7) yield expansion (7.11) proceeds in the same way as above, the only difference being that instead of (7.15) we now show that

$$
\begin{aligned}
\frac{1}{N} \sum_{j=1}^{N} E & \left(\Psi_{1}\left(U_{j: N}\right)-\Psi_{1}\left(\frac{j}{N+1}\right)\right)^{2} \\
& =\frac{1}{N+1} \sum_{j=1}^{N+1} E\left(\Psi_{1}\left(U_{j: N+1}\right)-\Psi_{1}\left(\frac{j}{N+2}\right)\right)^{2}+o\left(N^{-\frac{5}{3}}\right) .
\end{aligned}
$$

Using the recurrence relation for $b_{j, N}$ again, we find after some arithmetic

$$
\begin{aligned}
\frac{1}{N} \sum_{j=1}^{N} E & \left(\Psi_{1}\left(U_{j: N}\right)-\Psi_{1}\left(\frac{j}{N+1}\right)\right)^{2} \\
= & \frac{1}{N+1} \sum_{j=1}^{N+1} E\left(\Psi_{1}\left(U_{j: N+1}\right)-\Psi_{1}\left(\frac{j}{N+2}\right)\right)^{2} \\
& +\frac{2}{N+1} \sum_{j=1}^{N+1}\left\{\Psi_{1}\left(\frac{j}{N+2}\right)-\frac{j-1}{N} \Psi_{1}\left(\frac{j-1}{N+1}\right)\right. \\
& \left.-\frac{N-j+1}{N} \Psi_{1}\left(\frac{j}{N+1}\right)\right\} E\left(\Psi_{1}\left(U_{j: N+1}\right)-\Psi_{1}\left(\frac{j}{N+2}\right)\right) \\
& +\frac{1}{N+1} \sum_{j=1}^{N+1}\left\{\frac{j-1}{N}\left(\Psi_{1}\left(\frac{j}{N+2}\right)-\Psi_{1}\left(\frac{j-1}{N+1}\right)\right)^{2}\right. \\
& \left.+\frac{N-j+1}{N}\left(\Psi_{1}\left(\frac{j}{N+2}\right)-\Psi_{1}\left(\frac{j}{N+1}\right)\right)^{2}\right\}
\end{aligned}
$$

Now (5.18) ensures that

$$
\begin{aligned}
& \left|\Psi_{1}\left(\frac{j}{N+2}\right)-\Psi_{1}\left(\frac{j-1}{N+1}\right)\right| \leqq \tilde{M}\left(\frac{j}{N+2}\right) \frac{(N-j+2)}{(N+2)^{2}}\left\{\frac{j(N-j+2)}{(N+2)^{2}}\right\}^{-\frac{7}{6}} \\
& \text { for } \quad j=2, \cdots, N+1, \\
& \left|\Psi_{1}\left(\frac{j}{N+2}\right)-\Psi_{1}\left(\frac{j}{N+1}\right)\right| \leqq \tilde{M}\left(\frac{j}{N+2}\right) \frac{j}{(N+2)^{2}}\left\{\frac{j(N-j+2)}{(N+2)^{2}}\right\}^{-\frac{7}{6}} \\
& \text { for } j=1, \ldots, N,
\end{aligned}
$$


where $\tilde{M}$ is a bounded function on $(0,1)$ with $\lim _{t \rightarrow 0,1} \tilde{M}(t)=0$. Similarly, $(5.7)$, Lemma A2.3 in ABZ (1976) and (5.18) imply that

$$
\begin{aligned}
& \left|E \Psi_{1}\left(U_{j: N+1}\right)-\Psi_{1}\left(\frac{j}{N+2}\right)\right| \\
& \quad \leqq \tilde{M}_{2}\left(\frac{j}{N+2}\right) N^{-1}\left\{\frac{j(N-j+2)}{(N+2)^{2}}\right\}^{-\frac{7}{6}} \text { for } \quad j=1, \cdots, N+1 .
\end{aligned}
$$

It follows that both the second and third terms on the right in (7.18) are

$$
o\left(N^{-2} \int_{N^{-1}}^{1-N^{-1}}\{t(1-t)\}^{-\frac{4}{3}} d t\right)=o\left(N^{-\frac{5}{3}}\right),
$$

which proves (7.17) and therefore (7.11).

Finally, the validity of expansion (7.10) for exact as well as approximate scores is a simple consequence of (7.9) and (7.11) and the fact that Theorem 5.2 clearly implies that both $\sum \sigma^{2}\left(\Psi_{1}\left(U_{j: N}\right)\right)$ and $\sum E\left(\Psi_{1}\left(U_{j: N}\right)-\Psi_{1}(j /(N+1))\right)^{2}$ equal

$$
\int_{N^{-1}}^{1-N^{-1}}\left(\Psi_{1}^{\prime}(t)\right)^{2} t(1-t) d t+o(1)+O\left(N^{-\frac{1}{2}} \int_{N^{-1}}^{1-N^{-1}}\left(\Psi_{1}^{\prime}(t)\right)^{2}\{t(1-t)\}^{\frac{1}{2}} d t\right) .
$$

This completes the proof of the theorem.

Like Theorems 5.1 and 5.2, Theorem 7.1 presents us with a choice between an expansion with remainder $o(1)$ and one which is more explicit but may have a remainder of larger order under the conditions of the theorem. If $\Psi_{1}^{\prime}(t)=$ $o\left(\{t(1-t)\}^{-1}\right)$ for $t \rightarrow 0,1$, then $d_{N}=\tilde{d}_{N, 2}+o(1)$ for exact as well as approximate scores and expansion (7.10) is obviously preferable. This appears to be the most common case. However, if $\Psi_{1}^{\prime}(t)$ is of exact order $\{t(1-t)\}^{-1}$, then (7.10) yields only

$$
d_{N}=\frac{\int_{N^{-1}}^{1-N^{-1}}\left(\Psi_{1}^{\prime}(t)\right)^{2} t(1-t) d t}{\int_{0}^{1} \Psi_{1}^{2}(t) d t}+O(1)=O(\log N) .
$$

Finally, if $\Psi_{1}^{\prime}(t) \sim\{t(1-t)\}^{-1-\delta}$ for $t \rightarrow 0,1$ and some $0<\delta<\frac{1}{6}$, then $(7.10)$ reduces to $d_{N}=O\left(N^{2 \delta}\right)$.

In general, all we can say under the conditions of Theorem 7.1 is that

$$
d_{N}=\frac{\sum \sigma^{2}\left(\Psi_{1}\left(U_{j: N}\right)\right)}{\int \Psi_{1}^{2}(t) d t}+O(1)=O\left(\int_{N^{-1}}^{1-N^{-1}}\left(\Psi_{1}^{\prime}(t)\right)^{2} t(1-t) d t\right)=o\left(N^{\frac{1}{3}}\right)
$$

for exact scores, and that

$$
\begin{aligned}
d_{N} & =\frac{\sum E\left(\Psi_{1}\left(U_{j: N}\right)-\Psi_{1}(j /(N+1))\right)^{2}}{\int \Psi_{1}^{2}(t) d t}+O(1) \\
& =O\left(\int_{N^{-1}}^{1-N^{-1}}\left(\Psi_{1}^{\prime}(t)\right)^{2} t(1-t) d t\right)=o\left(N^{\frac{1}{3}}\right)
\end{aligned}
$$

for approximate scores. Even this result, however, is rather surprising because one might have expected these deficiencies to be of the order $N^{\frac{1}{2}}$. The reason that they are of smaller order than $N^{\frac{1}{2}}$ is of course that the power expansions for the rank tests in Theorem 5.2 and for the most powerful test in Lemma 7.2 agree not only in their leading terms of order 1 but also in their second order terms 
of order $N^{-\frac{1}{2}}$. It is only in third order terms that differences begin to show up. Borrowing a phrase from Pfanzag1 (1977), who noted the same phenomenon in the parametric one-sample problem, first order efficiency apparently implies second order efficiency in the cases considered. Note that results very similar to (7.19) and (7.20) were obtained for one-sample rank tests in ABZ (1976). In that case, however, there is no cause for surprise because certain symmetries that are present in the nonparametric one-sample problem ensure that there is no term of order $N^{-\frac{1}{2}}$ in any of the power expansions. Finally we should perhaps point out that in the present two-sample case, the fact that we have evaluated the envelope power for $\Delta_{0}$ as given in (7.5) instead of for the conventional choice $\Delta=\lambda$, is of no consequence for these considerations. For $\Delta=\lambda$ the term involving $\left(2 u_{\alpha}-\eta^{*}\right)^{2}$ should simply be omitted from (7.6) and (7.8) and this does not influence the qualitative behavior of $\tilde{\pi}^{+}$or $\tilde{d}_{N, i}$.

To provide some examples of Theorem 7.1 we compute the expansion (7.10) for the special case where $F$ is the logistic df $\Lambda(x)=\left(1+e^{-x}\right)^{-1}$ or the normal df $\Phi$. The computations resemble those at the end of Section 5. Suppose that $c \leqq \theta N^{\frac{1}{2}} \leqq C, \varepsilon \leqq \lambda \leqq 1-\varepsilon$ and $\varepsilon^{\prime} \leqq \alpha \leqq 1-\varepsilon^{\prime}$ for positive $c, C, \varepsilon$ and $\varepsilon^{\prime}$. As both examples concern symmetric distributions for which $\int \Psi_{1}{ }^{3}(t) d t=0$, the second order term in (7.5) vanishes so that we may take $\Delta_{0}=\lambda$ in both cases. For $F=\Lambda$ we are therefore concerned with the problem of testing the hypothesis $(\Lambda, \Lambda)$ against the alternative $(\Lambda(\cdot+\lambda \theta), \Lambda(\cdot-(1-\lambda) \theta))$ and $d_{N}$ denotes the deficiency of Wilcoxon's two-sample test with respect to the most powerful test for this problem. We find

$$
d_{N}=\frac{1}{20}\left[4 u_{\alpha}{ }^{2}+16+4 \eta^{*} u_{\alpha}+\frac{1-3 \lambda+3 \lambda^{2}}{\lambda(1-\lambda)} \eta^{* 2}\right]+o(1)
$$

with $\eta^{*}=\{\lambda(1-\lambda) N / 3\}^{\frac{1}{2}} \theta$. In this example $d_{N}$ remains bounded as $N \rightarrow \infty$.

In the second example we consider the testing problem $(\Phi, \Phi)$ versus $(\Phi(\cdot+\lambda \theta), \Phi(\cdot-(1-\lambda) \theta))$. Now $d_{N}$ is the deficiency of the two-sample normal scores test (or van der Waerden's two-sample test) with respect to the most powerful test based on the difference of the sample means. We obtain

$$
d_{N}=\log \log N+\frac{1}{2}\left(u_{\alpha}^{2}-3\right)+\log 2+\gamma+o(1)
$$

where $\gamma$ denotes Euler's constant (cf. (5.50)). Now $d_{N} \sim \log \log N \rightarrow \infty$ as $N \rightarrow \infty$. Note that there is no dependence on $\theta$ or $\lambda$ in this expansion.

So far in this section we have compared distributionfree tests to the most powerful test for a simple hypothesis against a simple alternative. However, all distributionfree tests occurring in this paper-rank tests as well as the permutaion test discussed in Section 6-are invariant under changes of location and scale. It would therefore be more realistic to compare these tests to the uniformly most powerful location and scale invariant test, if such a test exists. For the two-sample normal location problem Student's test answers this description and its power would therefore be a more suitable basis for comparison than 
the envelope power. For the problem of testing $(\Phi, \Phi)$ against $(\Phi(\cdot+\lambda \theta)$, $\Phi(\cdot-(1-\lambda) \theta))$ the power of the most powerful test equals $1-\Phi\left(u_{\alpha}-\eta^{*}\right)$ with $\eta^{*}=\{\lambda(1-\lambda) N\}^{\frac{1}{2}} \theta$. Assuming again that $c \leqq \theta N^{\frac{1}{2}} \leqq C, \varepsilon \leqq \lambda \leqq 1-\varepsilon$ and $\varepsilon^{\prime} \leqq \alpha \leqq 1-\varepsilon^{\prime}$ for positive $c, C, \varepsilon$ and $\varepsilon^{\prime}$, the power of Student's two-sample test is given by (6.35) and its deficiency with respect to the most powerful test is therefore equal to $\frac{1}{2} u_{\alpha}{ }^{2}+o(1)$. It follows from (7.22) that the deficiency of the two-sample normal scores test (or van der Waerden's two-sample test) with respect to Student's two-sample test for the normal location problem is given by

$$
d_{N}=\log \log N-\frac{3}{2}+\log 2+\gamma+o(1),
$$

where now the expansion does not even depend on $\alpha$. Since both tests are location invariant, (7.23) also denotes the deficiency for testing $(\Phi, \Phi)$ against $(\Phi, \Phi(\cdot-\theta))$.

We conclude this section by comparing the permutation test discussed in Section 6 to Student's test. Theorem 7.2 is an immediate consequence of Theorem 6.1, expansion (6.34) and (6.8).

THEOREM 7.2. Suppose that positive numbers $c, c^{\prime}, C, D, \varepsilon, \varepsilon^{\prime}, \delta$ and $r>8$ exist such that the conditions of Theorem 6.1 are satisfied and that $\theta \geqq c^{\prime} N^{-\frac{1}{2}}$. Let $d_{N}$ denote the deficiency of the permutation test based on the sample means with respect to Student's two-sample test for testing $(F, F)$ against $(F, F(\cdot-\theta))$ on the basis of $X_{1}, \cdots, X_{N}$ and at level $\alpha$. Then there exist $B>0$ depending only on $c, c^{\prime}, C, D, \varepsilon$, $\varepsilon^{\prime}$ and $\delta$, and $\beta>0$ depending only on $r$ such that

$$
d_{N} \leqq B N^{-\beta} .
$$

The case $F=\Phi$ is of course of most interest because then the theorem asserts that for the normal location problem there exists a distributionfree test whose deficiency with respect to the best location and scale invariant test tends to zero. We note that the remark at the end of Section 6 implies that in this case (7.24) may be replaced by $d_{N} \leqq B N^{-\frac{1}{2}}$. For $F \neq \Phi$ the theorem merely shows how closely the permutation test resembles Student's test with the correct significance level for $F$.

8. Expansions and deficiencies for related estimators. Let $X_{1}, \cdots, X_{N}$ be independent and let $(F, G)$ denote the hypothesis that $X_{1}, \cdots, X_{m}$ have common df $F$ and $X_{m+1}, \cdots, X_{N}$ have common df $G$. Let $T=T\left(X_{1}, \cdots, X_{N}\right)$ be the rank statistic given by (2.2) and suppose that the scores $a_{j}$ are nondecreasing in $j=$ $1, \cdots, N$. Define the statistic $M$ by

$$
\begin{aligned}
& M\left(X_{1}, \cdots, X_{N}\right) \\
& =\frac{1}{2} \sup \left\{t: T\left(X_{1}, \cdots, X_{m}, X_{m+1}-t, \cdots, X_{N}-t\right)>\lambda \sum a_{j}\right\} \\
& \quad+\frac{1}{2} \inf \left\{t: T\left(X_{1}, \cdots, X_{m}, X_{m+1}-t, \cdots, X_{N}-t\right)<\lambda \sum a_{j}\right\} .
\end{aligned}
$$

Under the model $(F, F(\bullet-\mu)), M$ was proposed as an estimator of $\mu$ by Hodges and Lehmann (1963). They showed that the normal approximation to the power 
of the level $\frac{1}{2}$ test based on $T$ for contiguous location alternatives can be used to establish asymptotic normality of $M$. In the same way we shall show that a power expansion yields an expansion for the df of $N^{\frac{1}{2}}(M-\mu)$. Note that we do not make the assumption of Hodges and Lehmann (1963) that the distribution of $T$ under $(F, F)$ is symmetric about $\lambda \sum a_{j}$, which occurs, e.g., when either $\lambda=\frac{1}{2}$ or the scores are antisymmetric. As a result the power expansion involved will be for the test based on $T$ at level $\bar{\alpha}=\frac{1}{2}+O\left(N^{-\frac{1}{2}}\right)$ rather than at level $\frac{1}{2}$, but for our deficiency computations this will not make any difference. We shall restrict attention to the case where $T$ is the statistic of the locally most powerful rank test or its approximate scores analogue, so that the $a_{j}$ will be exact or approximate scores generated by the score function $-\Psi_{1}$, with $\Psi_{1}$ as in (4.15). To ensure that the scores are nondecreasing we require that the density $f$ of $F$ is strongly unimodal, i.e., that $\log f$ is concave.

Let $\mathscr{F}$ be given by Definition 5.1 , let $\pi(\alpha, F, \theta)$ denote the power of the level $\alpha$ right-sided test based on $T$ against the alternative $(F, F(\cdot-\theta))$ and define

$$
\bar{\alpha}=\frac{1}{2}+\frac{(1-2 \lambda)}{6\{2 \pi \lambda(1-\lambda) N\}^{\frac{1}{2}}} \frac{\int \Psi_{1}^{3}(t) d t}{\left\{\int \Psi_{1}^{2}(t) d t\right\}^{\frac{3}{2}}} .
$$

Furthermore define, with $\Psi_{i}$ as in (4.15),

$$
\begin{aligned}
& \bar{L}_{0}(x)=\Phi(x)-\frac{\phi(x)}{288}\left[\frac{24(1-2 \lambda)}{\{\lambda(1-\lambda) N\}^{\frac{1}{2}}} \frac{\int \Psi_{1}{ }^{3}(t) d t}{\left\{\int \Psi_{1}^{2}(t) d t\right\}^{\frac{3}{2}}}\left(x^{2}+2\right)\right. \\
& -\frac{4}{\lambda(1-\lambda) N} \frac{\int \Psi_{1}^{4}(t) d t}{\left\{\int \Psi_{1}^{2}(t) d t\right\}^{2}} \\
& \times\left\{5\left(1-3 \lambda+3 \lambda^{2}\right) x^{3}-3\left(1-6 \lambda+6 \lambda^{2}\right) x\right\} \\
& +\frac{48\left(1-3 \lambda+3 \lambda^{2}\right)}{\lambda(1-\lambda) N} \frac{\int \Psi_{2}^{2}(t) d t}{\left\{\int \Psi_{1}^{2}(t) d t\right\}^{2}} x^{3} \\
& +\frac{(1-2 \lambda)^{2}}{\lambda(1-\lambda) N} \frac{\left\{\int \Psi_{1}^{3}(t) d t\right\}^{2}}{\left\{\int \Psi_{1}^{2}(t) d t\right\}^{3}}\left(x^{5}-4 x^{3}-12 x\right) \\
& \left.-\frac{36}{\lambda(1-\lambda) N}\left\{\left(1-3 \lambda+3 \lambda^{2}\right) x^{3}+x\right\}\right] \text {, } \\
& \bar{L}_{1}(x)=\bar{L}_{0}(x)-\frac{x \phi(x)}{2 N \int \Psi_{1}^{2}(t) d t} \sum_{j=1}^{N} \sigma^{2}\left(\Psi_{1}\left(U_{j: N}\right)\right), \\
& \bar{L}_{2}(x)=\bar{L}_{0}(x)-\frac{x \phi(x)}{2 N \int \Psi_{1}^{2}(t) d t} \int_{N^{-1}}^{1-N-1}\left(\Psi_{1}^{\prime}(t)\right)^{2} t(1-t) d t, \\
& \bar{L}_{3}(x)=\bar{L}_{0}(x)-\frac{x \phi(x)}{2 N \int \Psi_{1}^{2}(t) d t} \sum_{j=1}^{N} E\left(\Psi_{1}\left(U_{j: N}\right)-\Psi_{1}\left(\frac{j}{N+1}\right)\right)^{2} .
\end{aligned}
$$

Probabilities under the model $(F, F(\cdot-\mu))$ are denoted by $P_{F, \mu}$.

THEOREM 8.1. Suppose that $F \in F$, that $f$ is strongly unimodal and that either $a_{j}=-E \Psi_{1}\left(U_{j: N}\right)$ for $j=1, \cdots, N$, or $a_{j}=-\Psi(j /(N+1))$ for $j=1, \cdots, N$. Let $\varepsilon$ and $C$ be positive numbers and suppose that $\varepsilon \leqq \lambda \leqq 1-\varepsilon$. Then there exist positive 
numbers $B, \delta_{1}, \delta_{2}, \cdots$, with $\lim _{N \rightarrow \infty} \delta_{N}=0$, which depend only on $F, \varepsilon$ and $C$, such that

$$
\sup _{|\xi| \leqq C}\left|P_{F, \mu}\left(N^{\frac{1}{2}}(M-\mu) \leqq \xi\right)-\left\{1-\pi\left(\bar{\alpha}, F,-\xi N^{-\frac{1}{2}}\right)\right\}\right| \leqq \delta_{N} N^{-1}
$$

and such that the following statements hold:

(i) for exact scores $a_{j}=-E \Psi_{1}\left(U_{j: N}\right)$,

$$
\begin{gathered}
\sup _{|x| \leqq C}\left|P_{F, \mu}\left(\left\{\lambda(1-\lambda) N \int \Psi_{1}{ }^{2}(t) d t\right\}^{\frac{1}{2}}(M-\mu) \leqq x\right)-\bar{L}_{1}(x)\right| \leqq \delta_{N} N^{-1} \\
\sup _{|x| \leqq C}\left|P_{F, \mu}\left(\left\{\lambda(1-\lambda) N \int \Psi_{1}{ }^{2}(t) d t\right\}^{\frac{1}{2}}(M-\mu) \leqq x\right)-\bar{L}_{2}(x)\right| \\
\leqq \delta_{N} N^{-1}+B N^{-\frac{3}{2}} \int_{N^{-1}}^{1-N-1}\left(\Psi_{1}{ }^{\prime}(t)\right)^{2}\{t(1-t)\}^{\frac{1}{2}} d t
\end{gathered}
$$

(ii) for approximate scores $a_{j}=-\Psi_{1}(j /(N+1))$,

$$
\sup _{|x| \leqq C}\left|P_{F, \mu}\left(\left\{\lambda(1-\lambda) N \int \Psi_{1}^{2}(t) d t\right\}^{\frac{1}{2}}(M-\mu) \leqq x\right)-\bar{L}_{3}(x)\right| \leqq \delta_{N} N^{-1}
$$

and (8.6) continues to hold.

Proof. In view of $(8.1)$ we have for $\theta=-\xi N^{-\frac{1}{2}}$,

$$
\begin{gathered}
P_{F, \mu}\left(N^{\frac{1}{2}}(M-\mu) \leqq \xi\right)=P_{F, \theta}(M \leqq 0) \\
P_{F, \theta}\left(T<\lambda \sum a_{j}\right) \leqq P_{F, \theta}(M \leqq 0) \leqq P_{F, \theta}\left(T \leqq \lambda \sum a_{j}\right) .
\end{gathered}
$$

For $\theta=-\xi N^{-\frac{1}{2}}$ and $\varepsilon^{\prime} \leqq \alpha \leqq 1-\varepsilon^{\prime}$, the conditions of Theorem 5.2 are satisfied except, of course, that $\theta<0$ if $\xi>0$. However, the theorem remains valid for $|\theta| \leqq D N^{-\frac{1}{2}}$; it was formulated for positive $\theta$ merely because we were discussing one-sided tests against one-sided alternatives at that point. It follows that $P_{F, \theta}\left(T=\lambda \sum a_{j}\right)=o\left(N^{-1}\right)$ uniformly for $|\xi| \leqq C$, so that

$$
\begin{aligned}
P_{F, \mu}\left(N^{\frac{1}{2}}(M-\mu) \leqq \xi\right) & =P_{F, \theta}\left(T \leqq \lambda \sum a_{j}\right)+o\left(N^{-1}\right) \\
& =1-\pi\left(\alpha, F,-\xi N^{-\frac{1}{2}}\right)+o\left(N^{-1}\right),
\end{aligned}
$$

where $\alpha$ is the level of the test that rejects if $T>\lambda \sum a_{j}$. Noting that $\sum a_{j}=0$ for exact scores, we find from (5.33) and (5.37) for $x=\eta^{*}=0$, that $\alpha=\bar{\alpha}+o\left(N^{-1}\right)$. In view of (5.35) and (5.39) this yields $\pi\left(\alpha, F,-\xi N^{-\frac{1}{2}}\right)=$ $\pi\left(\bar{\alpha}, F,-\xi N^{-\frac{1}{2}}\right)+o\left(N^{-1}\right)$ uniformly for $|\xi| \leqq C$ and together with (8.8) this proves (8.4). The remainder of the theorem follows from (8.8) and expansions (5.33), (5.34), (5.37) and (5.38) with $x$ and $\eta^{*}$ replaced by 0 and $-x$.

The natural parametric competitor of $M$ as an estimator of $\mu$ is of course the maximum likelihood estimator $M^{\prime}$. Under the model $K_{F, \mu, \Delta}=(F(\cdot+\Delta \mu)$, $F(\cdot-(1-\Delta) \mu)), M^{\prime}=M_{\Delta}^{\prime}$ is the solution of

$$
\Delta \sum_{i=1}^{m} \psi_{1}\left(X_{i}+\Delta M^{\prime}\right)-(1-\Delta) \sum_{i=m+1}^{N} \psi_{1}\left(X_{i}-(1-\Delta) M^{\prime}\right)=0
$$

with $\psi_{1}=f^{\prime} / f$ as in (4.1). Note that, in contrast to $M$, the estimator $M_{\Delta}{ }^{\prime}$ as well as its distribution under $K_{F, \mu, \Delta}$ depend on $\Delta$.

The df of $M_{\Delta}^{\prime}$ under $K_{F, \mu, \Delta}$ is connected with the power of the locally most powerful test for $H_{F}=(F, F)$ against $K_{F, \theta, \Delta}$. For $\theta>0$, this test rejects $H_{F}$ for 
large values of the statistic

$$
S_{\Delta}=\Delta \sum_{i=1}^{m} \psi_{1}\left(X_{i}\right)-(1-\Delta) \sum_{i=m+1}^{N} \psi_{1}\left(X_{i}\right) .
$$

Let $\pi(\alpha, F, \theta, \Delta)$ denote the power against $K_{F, \theta, \Delta}$ of this right-sided test at level $\alpha$. Suppose that $F$ is a fixed df with density $f$ that is positive and five times differentiable on $R^{1}$ and define

$$
\begin{aligned}
\tilde{\pi}(\alpha, F, \theta, \Delta) & \\
=1 & -\Phi\left(u_{\alpha}-\tilde{\eta}\right)-\frac{\tilde{\eta} \phi\left(u_{\alpha}-\tilde{\eta}\right)}{288}\left[\frac{24}{N^{\frac{1}{2}}} \frac{\tau_{3} \int \Psi_{1}^{3}(t) d t}{\left\{\tau_{2} \int \Psi_{1}^{2}(t) d t\right\}^{\frac{3}{2}}}\left(-2 u_{\alpha}+\tilde{\eta}\right)\right. \\
& +\frac{4}{N} \frac{\tau_{4} \int \Psi_{1}{ }^{4}(t) d t}{\left\{\tau_{2} \int \Psi_{1}^{2}(t) d t\right\}^{2}}\left\{-3\left(u_{\alpha}{ }^{2}-1\right)+3 \tilde{\eta} u_{\alpha}-5 \tilde{\eta}^{2}\right\} \\
& +\frac{48}{N} \frac{\tau_{4} \int \Psi_{2}{ }^{2}(t) d t}{\left\{\tau_{2} \int \Psi_{1}^{2}(t) d t\right\}^{2}} \tilde{\eta}^{2}+\frac{1}{N} \frac{\left\{\tau_{3} \int \Psi_{1}^{3}(t) d t\right\}^{2}}{\left\{\tau_{2} \int \Psi_{1}^{2}(t) d t\right\}^{3}} \\
& \times\left\{8\left(2 u_{\alpha}{ }^{2}-1\right)-4 \tilde{\eta}\left(u_{\alpha}{ }^{3}+3 u_{\alpha}\right)+8 \tilde{\eta}^{2}\left(u_{\alpha}{ }^{2}-1\right)-5 \tilde{\eta}^{3} u_{\alpha}+\tilde{\eta}^{4}\right\} \\
& \left.+\frac{36 \tau_{4}}{N \tau_{2}{ }^{2}}\left\{\left(u_{\alpha}{ }^{2}-1\right)-\tilde{\eta} u_{\alpha}-\tilde{\eta}^{2}\right\}\right],
\end{aligned}
$$

where $\tilde{\eta}$ and $\tau_{k}$ are given by (7.2) and (7.3).

LeMma 8.1. Let $F$ satisfy (5.6) for $m_{i}=5 / i, i=1, \cdots, 5$, and suppose that positive numbers $D, D^{\prime}$, $\varepsilon$ and $\varepsilon^{\prime}$ exist such that $|\theta| \leqq D N^{-\frac{1}{2}},|\Delta \theta| \leqq D^{\prime} N^{-\frac{1}{2}}, \varepsilon \leqq \lambda \leqq$ $1-\varepsilon$ and $\varepsilon^{\prime} \leqq \alpha \leqq 1-\varepsilon^{\prime}$. Then there exists $B>0$ depending only on $F, D, D^{\prime}$, $\varepsilon$ and $\varepsilon^{\prime}$ such that

$$
|\pi(\alpha, F, \theta, \Delta)-\tilde{\pi}(\alpha, F, \theta, \Delta)| \leqq B N^{-\frac{3}{2}} .
$$

Proof. The proof proceeds in the same manner as that of Lemma 7.1 and again we omit the details. Under the conditions of the lemma we find that under $K_{F, \theta, \Delta}$,

$$
\begin{aligned}
& P\left(\frac{S_{\Delta}}{\left\{N \tau_{2} \int \Psi_{1}^{2}(t) d t\right\}^{\frac{1}{2}}} \leqq x\right) \\
& =\Phi(x-\tilde{\eta})-\frac{\phi(x-\tilde{\eta})}{288}\left[\frac{24}{N^{\frac{1}{2}}} \frac{\tau_{3} \int \Psi_{1}{ }^{3}(t) d t}{\left\{\Psi_{1}{ }^{2}(t) d t\right\}^{\frac{3}{2}}}\left\{2\left(x^{2}-1\right)+2 \tilde{\eta} x-\tilde{\eta}^{2}\right\}\right. \\
& \quad+\frac{4}{N} \frac{\tau_{4} \int \Psi_{1}^{4}(t) d t}{\left\{\tau_{2} \int \Psi_{1}^{2}(t) d t\right\}^{2}}\left\{3\left(x^{3}-3 x\right)+3 \tilde{\eta}\left(x^{2}-1\right)-3 \tilde{\eta}^{2} x+5 \tilde{\eta}^{3}\right\} \\
& \quad-\frac{48}{N} \frac{\tau_{4} \int \Psi_{2}^{2}(t) d t}{\left\{\tau_{2} \int \Psi_{1}^{2}(t) d t\right\}^{2}} \tilde{\eta}^{3}+\frac{1}{N} \frac{\left\{\tau_{3} \int \Psi_{1}^{3}(t) d t\right\}^{2}}{\left\{\tau_{2} \int \Psi_{1}^{2}(t) d t\right\}^{3}}\left\{4\left(x^{5}-10 x^{3}+15 x\right)\right. \\
& \left.\quad+4 \tilde{\eta}\left(x^{4}-6 x^{2}+3\right)-8 \tilde{\eta}^{2}\left(x^{3}-3 x\right)-4 \tilde{\eta}^{3}\left(x^{2}-1\right)+5 \tilde{\eta}^{4} x-\tilde{\eta}^{5}\right\} \\
& \left.\quad+\frac{36 \tau_{4}}{N \tau_{2}^{2}}\left\{-\left(x^{3}-3 x\right)-\tilde{\eta}\left(x^{2}-1\right)+\tilde{\eta}^{2} x+\tilde{\eta}^{3}\right\}\right]+O\left(N^{-\frac{3}{2}}\right) .
\end{aligned}
$$

The remainder term is uniformly $O\left(N^{-\frac{3}{2}}\right)$ for fixed $F, D, D^{\prime}, \varepsilon$ and $\varepsilon^{\prime}$. This expansion yields $(8.12)$.

Note that the expansions (8.12) and (8.13) are valid also for negative values 
of $\theta$, but that the right-sided test considered here is not locally most powerful against these alternatives.

If the conditions of Lemma 8.1 are fulfilled and if, moreover, $f$ is strongly unimodal so that $\psi_{1}$ is nonincreasing, then we can establish the connection between $\pi(\alpha, F, \theta, \Delta)$ and the $\mathrm{df}$ of $M_{\Delta}^{\prime}$ by arguing as in the proof of Theorem 8.1. Writing $P_{F, \mu, \Delta}$ for probabilities under $K_{F, \mu, \Delta}$ and taking $\theta=-\xi N^{-\frac{1}{2}}$ we find that

$$
P_{F, \theta, \Delta}\left(S_{\Delta}<0\right) \leqq P_{F, \mu, \Delta}\left(N^{\frac{1}{2}}\left(M_{\Delta}{ }^{\prime}-\mu\right) \leqq \xi\right) \leqq P_{F, \theta, \Delta}\left(S_{\Delta} \leqq 0\right) .
$$

In view of (8.12) and (8.13) this implies that uniformly for $|\xi|,|x| \leqq C$,

$$
\begin{aligned}
& P_{F, \mu, \Delta}\left(N^{\frac{1}{2}}\left(M_{\Delta}^{\prime}-\mu\right) \leqq \xi\right)=1-\pi(\tilde{\alpha}, F, \theta, \Delta)+O\left(N^{-\frac{3}{2}}\right), \\
& \tilde{\alpha}=\frac{1}{2}-\frac{1}{6\{2 \pi N\}^{\frac{1}{2}}} \frac{\tau_{3} \int \Psi_{1}^{3}(t) d t}{\left\{\tau_{2} \int \Psi_{1}^{2}(t) d t\right\}^{\frac{3}{2}}}, \\
& P_{F, \mu, \Delta}\left(\left\{\tau_{2} N \int \Psi_{1}^{2}(t) d t\right\}^{\frac{1}{2}}\left(M_{\Delta}^{\prime}-\mu\right) \leqq x\right) \\
& =\Phi(x)-\frac{\phi(x)}{288}\left[-\frac{24}{N^{\frac{1}{2}}} \frac{\tau_{3} \int \Psi_{1}^{3}(t) d t}{\left\{\tau_{2} \int \Psi_{1}^{2}(t) d t\right\}^{\frac{3}{2}}}\left(x^{2}+2\right)\right. \\
& -\frac{4}{N} \frac{\tau_{4} \int \Psi_{1}^{4}(t) d t}{\left\{\tau_{2} \int \Psi_{1}^{2}(t) d t\right\}^{2}}\left(5 x^{3}-3 x\right)+\frac{48}{N} \frac{\tau_{4} \int \Psi_{2}^{2}(t) d t}{\left\{\tau_{2} \int \Psi_{1}^{2}(t) d t\right\}^{2}} x^{3} \\
& \left.+\frac{1}{N} \frac{\left\{\tau_{3} \int \Psi_{1}^{3}(t) d t\right\}^{2}}{\left\{\tau_{2} \int \Psi_{1}^{2}(t) d t\right\}^{3}}\left(x^{5}-4 x^{3}-12 x\right)-\frac{36 \tau_{4}}{N \tau_{2}{ }^{2}}\left(x^{3}+x\right)\right] \\
& +O\left(N^{-\frac{3}{2}}\right) \text {. }
\end{aligned}
$$

We have already remarked that the $\mathrm{df}$ of $\left(M_{\Delta}^{\prime}-\mu\right)$ under $K_{F, \mu, \Delta}$ depends on $\Delta$ and thus the same problem arises that we encountered in Section 7, viz. to determine the "right" $\Delta$ for which $M$ and $M^{\prime}$ should be compared. It is easy to see from (8.16) that the value $\Delta=\Delta^{0}$ that is least favorable for $M^{\prime}$ in the sense that it minimizes (maximizes) $P_{F, \mu, \Delta}\left(\left\{N \lambda(1-\lambda) \int \Psi_{1}^{2}(t) d t\right\}^{\frac{1}{2}}\left(M_{\Delta}^{\prime}-\mu\right) \leqq x\right)$ for positive (negative) $x$ is given by

$$
\Delta^{0}=\lambda-\frac{\{\lambda(1-\lambda)\}^{\frac{1}{2}}}{4 N^{\frac{1}{2}}} \frac{\int \Psi_{1}^{3}(t) d t}{\left\{\int \Psi_{1}^{2}(t) d t\right\}^{\frac{3}{2}}} \frac{x^{2}+2}{x}+O\left(N^{-1}\right) .
$$

However, we shall not take $\Delta=\Delta^{0}$ as a basis for comparing $M$ and $M^{\prime}$ but we shall simply choose $\Delta=\lambda$ instead. We advance three reasons for doing so. The reader who does not find these reasons sufficiently compelling should realize that we are merely granting the maximum likelihood estimator a slight additional advantage.

(i) The second order term of $\Delta^{0}$ depends on $x$ just as the second order term of $\Delta_{0}$ in (7.5) depends on $\theta$. This did not deter us from choosing $\Delta=\Delta_{0}$ as a basis for comparison in Section 7 , but we feel the situation is slightly different there. In Section 7 we were comparing with envelope power and in general this means comparing with a different most powerful test for each alternative $(\theta, \Delta)$. This being so, there seems to be little reason not to choose the least favorable testing problem for each value of $\theta$, i.e., to take $\Delta=\Delta_{0}$. All we are doing is 
locating a curve $\left(\theta, \Delta_{0}(\theta)\right)$ of least favorable alternatives in the set of all alternatives $(\theta, \Delta)$ and comparing with envelope power on that curve only.

Our attitude would have been different, however, if in Section 7 we had been comparing with the power of the locally most powerful test rather than with the envelope power. The locally most powerful test is of course independent of $\theta$ (cf. (8.10)) and for every fixed $\Delta$ we would therefore be comparing with a single fixed test for all $\theta$. In this case it would still be reasonable to choose $\Delta=\lambda$ which is least favorable to first order, but if $\int \Psi_{1}{ }^{3}(t) d t \neq 0$, it would seem to be rather extreme to compute the power of the locally most powerful test at each $\theta$ for $\Delta=\Delta_{0}=\Delta_{0}(\theta)$ which is least favorable to second order in this case too. After all, for every fixed $\Delta$ there would be a single locally most powerful test that does better than that for all values of $\theta$ except the one for which $\Delta_{0}(\theta)=\Delta$. It is precisely for such sets of alternatives ( $\Delta$ fixed, $\theta$ unknown) that the locally most powerful test is designed and it seems unrealistic to assess its performance only for a different one-parameter set of alternatives $\left(\theta, \Delta_{0}(\theta)\right)$.

The present problem for the maximum likelihood estimator is of course very similar to the one for the locally most powerful test. Again the choice $\Delta=\Delta^{0}$ depending on $x$ appears to be rather extreme because for every $\Delta$ the df of the maximum likelihood estimator is more concentrated around $\mu$ than this choice would indicate at all but at most two points.

(ii) Even though, in general, the distribution of $M_{\Delta}{ }^{\prime}$ under $K_{F, \mu, \Delta}$ is not symmetric about $\mu$, most reasonable measures of dispersion are built around the distribution of $\left|M_{\Delta}^{\prime}-\mu\right|$ rather than $\left(M_{\Delta}^{\prime}-\mu\right)$. It is clear from (8.16) that $P_{F, \mu, \Delta}\left(\left\{N \lambda(1-\lambda) \int \Psi_{1}^{2}(t) d t\right\}^{\frac{1}{2}}\left|M_{\Delta}^{\prime}-\mu\right| \leqq x\right)$ is minimized by $\Delta=\lambda+O\left(N^{-1}\right)$; it is also obvious from (8.16) that it makes no difference for our asymptotic results if we take $\Delta=\lambda$ instead (cf. the remark following (7.5)). Hence $\Delta=\lambda$ is the "right" choice of $\Delta$ for our asymptotic comparison of $M$ and $M^{\prime}$, provided that the comparison is made on the basis of the distributions of $|M-\mu|$ and $\left|M^{\prime}-\mu\right|$.

(iii) Our final argument is the rather more pedestrain one that any choice of $\Delta$ other than $\Delta=\lambda+o\left(N^{-\frac{1}{2}}\right)$ would to a certain extent destroy the simplicity of the main results in this section. We shall elaborate points (ii) and (iii) after proving Theorem 8.3.

We now substitute $\Delta=\lambda$ in $(8.14)-(8.16)$ and find that $\tilde{\alpha}$ reduces to $\bar{\alpha}$ as defined in (8.2) and that the expansion on the right in (8.16) becomes

$$
\begin{aligned}
L^{*}(x)=\Phi(x) & -\frac{\phi(x)}{288}\left[\frac{24(1-2 \lambda)}{\{\lambda(1-\lambda) N\}^{\frac{1}{2}}} \frac{\int \Psi_{1}{ }^{3}(t) d t}{\left\{\int \Psi_{1}^{2}(t) d t\right\}^{\frac{3}{2}}}\left(x^{2}+2\right)\right. \\
& -\frac{4\left(1-3 \lambda+3 \lambda^{2}\right)}{\lambda(1-\lambda) N} \frac{\int \Psi_{1}^{4}(t) d t}{\left\{\int \Psi_{1}^{2}(t) d t\right\}^{2}}\left(5 x^{3}-3 x\right)+\frac{48\left(1-3 \lambda+3 \lambda^{2}\right)}{\lambda(1-\lambda) N} \\
& \times \frac{\int \Psi_{2}^{2}(t) d t}{\left\{\int \Psi_{1}^{2}(t) d t\right\}^{2}} x^{3}+\frac{(1-2 \lambda)^{2}}{\lambda(1-\lambda) N} \frac{\left\{\int \Psi_{1}^{3}(t) d t\right\}^{2}}{\left\{\int \Psi_{1}^{2}(t) d t\right\}^{3}}\left(x^{5}-4 x^{3}-12 x\right) \\
& \left.-\frac{36\left(1-3 \lambda+3 \lambda^{2}\right)}{\lambda(1-\lambda) N}\left(x^{3}+x\right)\right] .
\end{aligned}
$$


We have proved

Theorem 8.2. Suppose that $F$ satisfies (5.6) for $m_{i}=5 / i, i=1, \ldots, 5$ and that $f$ is strongly unimodal. Let $\varepsilon$ and $C$ be positive numbers and suppose that $\varepsilon \leqq \lambda \leqq$ $1-\varepsilon$. Then there exists $B>0$ depending only on $F, \varepsilon$ and $C$, such that

$$
\begin{aligned}
& \sup _{|\xi| \leqq C}\left|P_{F, \mu, \lambda}\left(N^{\frac{1}{2}}\left(M_{\lambda}^{\prime}-\mu\right) \leqq \xi\right)-\left\{1-\pi\left(\bar{\alpha}, F,-\xi N^{-\frac{1}{2}}, \lambda\right)\right\}\right| \leqq B N^{-\frac{3}{2}}, \\
& \sup _{|x| \leqq C}\left|P_{F, \mu, \lambda}\left(\left\{\lambda(1-\lambda) N \int \Psi_{1}^{2}(t) d t\right\}^{\frac{1}{2}}\left(M_{\lambda}^{\prime}-\mu\right) \leqq x\right)-L^{*}(x)\right| \leqq B N^{-\frac{3}{2}} .
\end{aligned}
$$

There is no unique natural measure to assess the performance of the estimators $M$ and $M_{\lambda}^{\prime}$ on the basis of the expansions (8.5)-(8.7) and (8.19) and consequently there is no unique natural definition of the deficiency of $M$ with respect to $M_{\lambda}^{\prime}$ either. Let us, for a moment, indicate the dependence on the sample size $N$ in our notation and write $M_{N}$ and $M_{\lambda, N}^{\prime}$ for $M$ and $M_{\lambda}{ }^{\prime}$. For any real $\xi$ we define the deficiency $D_{N}(\xi)$ of the sequence of estimators $\left\{M_{N}\right\}$ with respect to the estimator $M_{\lambda, N}^{\prime}$ by equating the df's of $\left(M_{N+D_{N}}-\mu\right)$ under $P_{F, \mu, \lambda}\left(\right.$ or $\left.P_{F, \mu}\right)$ and of $\left(M_{\lambda, N}^{\prime}-\mu\right)$ under $P_{F, \mu, \lambda}$ at the point $\xi N^{-\frac{1}{2}}$, thus

$$
P_{F, \mu}\left(M_{N+D_{N}}-\mu \leqq \xi N^{-\frac{1}{2}}\right)=P_{F, \mu, \lambda}\left(M_{\lambda, N}^{\prime}-\mu \leqq \xi N^{-\frac{1}{2}}\right),
$$

with the usual convention that the probability on the left is defined by linear interpolation for nonintegral values of $N+D_{N}$. Of course, one will normally not be inclined to judge the performance of $\left\{M_{N}\right\}$ with respect to $M_{\lambda, N}^{\prime}$ on the basis of $D_{N}(\xi)$ for one value of $\xi$ only, but rather on the behavior of $D_{N}(\xi)$ as a function of $\xi$. In our asymptotic study this will not make any difference because the expansions for $D_{N}(\xi)$ will be found to be independent of $\xi$.

Turning to the corresponding tests, we let $d_{N}(\alpha, \theta)$ denote the deficiency in the usual sense of the locally most powerful rank test (or its approximate scores version) with respect to the locally most powerful test for the problem of testing $H_{F}=(F, F)$ against $K_{F, \theta, \lambda}=(F(\cdot+\lambda \theta), F(\cdot-(1-\lambda) \theta))$ at level $\alpha$. Since we shall be concerned with negative as well as positive values of $\theta$, we note that for positive (negative) $\theta$ the tests involved reject $H_{F}$ for large (small) values of the statistics given in (2.2) and (8.10), where the scores in (2.2) are exact or approximate scores generated by $-\Psi_{1}$.

Let $\mathscr{F}_{1}$ be given by Definition 7.1 and define

$$
\begin{aligned}
\tilde{D}_{N, 1}= & -\frac{1}{4} \frac{\int \Psi_{1}^{4}(t) d t}{\left\{\int \Psi_{1}^{2}(t) d t\right\}^{2}}-\frac{3}{4}+\frac{1}{\int \Psi_{1}^{2}(t) d t} \sum_{j=1}^{N} \sigma^{2}\left(\Psi_{1}\left(U_{j: N}\right)\right) \\
\tilde{D}_{N, 2}= & -\frac{1}{4} \frac{\int \Psi_{1}^{4}(t) d t}{\left\{\int \Psi_{1}^{2}(t) d t\right\}^{2}}-\frac{3}{4}+\frac{1}{\int \Psi_{1}^{2}(t) d t} \int_{N^{-1}}^{1-N^{-1}}\left(\Psi_{1}^{\prime}(t)\right)^{2} t(1-t) d t \\
\tilde{D}_{N, 3}=-\frac{1}{4} \frac{\int \Psi_{1}^{4}(t) d t}{\left\{\int \Psi_{1}^{2}(t) d t\right\}^{2}}-\frac{3}{4} & \\
& +\frac{1}{\int \Psi_{1}^{2}(t) d t} \sum_{j=1}^{N} E\left(\Psi_{1}\left(U_{j: N}\right)-\Psi_{1}\left(\frac{j}{N+1}\right)\right)^{2} .
\end{aligned}
$$

THEOREM 8.3. Let $d_{N}(\alpha, \theta)$ be the deficiency of the locally most powerful rank test with respect to the locally most powerful test for testing $H_{F}$ against $K_{F, \theta, \lambda}$ at 
level $\alpha$. Let $D_{N}(\xi)$ be the deficiency of the Hodges-Lehmann estimator associated with the locally most powerful rank test with respect to the maximum likelihood estimator for estimating $\mu$ under $K_{F, \mu, \lambda}$. Suppose that $F \in F_{1}$ and that $f$ is strongly unimodal. Let $c, C$ and $\varepsilon$ be positive numbers and suppose that $c \leqq|\xi| \leqq C$ and $\varepsilon \leqq \lambda \leqq 1-\varepsilon$. Then there exist positive numbers $B, \delta_{1}, \delta_{3}, \cdots$, with $\lim _{N \rightarrow \infty} \delta_{N}=0$, which depend only on $F, c, C$ and $\varepsilon$, such that

$$
\begin{gathered}
\left|D_{N}(\xi)-d_{N}\left(\frac{1}{2},-\xi N^{-\frac{1}{2}}\right)\right| \leqq \delta_{N}, \\
\left|D_{N}(\xi)-\tilde{D}_{N, 1}\right| \leqq \delta_{N}, \\
\left|D_{N}(\xi)-\tilde{D}_{N, 2}\right| \leqq \delta_{N}+B N^{-\frac{1}{2}} \int_{N^{-1}}^{1-1}\left(\Psi_{1}^{\prime}(t)\right)^{2}\{t(1-t)\}^{\frac{1}{2}} d t .
\end{gathered}
$$

If in the locally most powerful rank test and in the associated estimator, the exact scores are replaced by the approximate scores $a_{j}=-\Psi_{1}(j /(N+1))$, then $(8.22)$ and (8.24) remain valid and (8.23) is replaced by

$$
\left|D_{N}(\xi)-\tilde{D}_{N, 3}\right| \leqq \delta_{N}
$$

PROOF. Since $\mathscr{F}_{1} \subset \mathscr{F}$, the conditions of Theorems 8.1 and 8.2 are satisfied and (8.5)-(8.7) and (8.19) provide expansions for the df's of the estimators considered. Substituting the appropriate expansions in (8.20) and proceeding exactly as in the proof of Theorem 7.1, we arrive at (8.23) and (8.24) for the estimator associated with the locally most powerful rank test and at (8.24) and (8.25) for its approximate scores version.

Turning to the corresponding tests, (8.4) and (8.18) clearly imply that for negative values of $\xi$ the computation for obtaining an expansion for $d_{N}\left(\bar{\alpha},-\xi N^{-\frac{1}{2}}\right)$ is precisely the same as for $D_{N}(\xi)$. In view of (8.23) and (8.25) this computation determines the deficiency up to $o(1)$ and hence

$$
\left|D_{N}(\xi)-d_{N}\left(\bar{\alpha},-\xi N^{-\frac{1}{2}}\right)\right|=o(1), \quad \text { for } \quad-C \leqq \xi \leqq-c .
$$

For positive $\xi, d_{N}\left(\alpha,-\xi N^{-\frac{1}{2}}\right)$ refers to testing for negative shift and therefore to the left-sided tests rather than the right-sided tests whose powers appear in (8.4) and (8.18). Since the powers of the left-and right-sided versions of a test sum to 1 if their significance levels do, we find

$$
\left|D_{N}(\xi)-d_{N}\left(1-\bar{\alpha},-\xi N^{-\frac{1}{2}}\right)\right|=o(1), \quad \text { for } \quad c \leqq \xi \leqq C .
$$

Note that (8.26) and (8.27) hold for exact as well as approximate scores and that the remainder terms are uniformly $o(1)$ for fixed $F, c, C$ and $\varepsilon$.

It remains to show that $\bar{\alpha}$ may be replaced by $\frac{1}{2}$ in $(8.26)$ and (8.27). If we take $\Delta=\lambda$ in the power expansion for the locally most powerful test in Lemma 8.1 and compare the result with the power expansion for the most powerful test in Lemma 7.2, we see that the terms of orders 1 and $N^{-\frac{1}{2}}$ agree and that in the terms of order $N^{-1}$ only certain coefficients differ. Moreover, for $\Delta=\lambda$ the conditions of Lemma 8.1 are identical with those of Lemma 7.2. This means that if we replace the most powerful test by the locally most powerful test in Theorem 7.1, then the theorem will remain valid if some of the coefficients in 
$\tilde{d}_{N, 0}$ are changed. Thus, under the conditions of Theorem 7.1 there exists, for exact as well as approximate scores, an expansion for $d_{N}(\alpha, \theta)$ with a bounded derivative with respect to $\alpha$ and a remainder term $o(1)$. This statement remains correct for $-C N^{-\frac{1}{2}} \leqq \theta \leqq-c N^{-\frac{1}{2}}$ because the power expansions in Lemma 8.1 and Theorem 5.2 are valid for negative $\theta$ too (cf. the remark in the proof of Theorem 8.1) so that the only change in the expansion for $d_{N}(\alpha, \theta)$ is a change of sign of $u_{\alpha}$ to account for the switch from the right-sided to the left-sided tests. Noting that $c \leqq|\xi| \leqq C$ and that $\bar{\alpha}=\frac{1}{2}+O\left(N^{-\frac{1}{2}}\right)$ we find that we may indeed replace $\bar{\alpha}$ by $\frac{1}{2}$ in (8.26) and (8.27) without affecting the right-hand side and its uniformity for fixed $F, c, C$ and $\varepsilon$. This proves $(8.22)$ and the theorem.

A number of comments should be made at this point. First of all we recall remarks (ii) and (iii) in our discussion earlier in this section concerning the choice of $\Delta$ for which $M$ and $M^{\prime}$ should be compared. Suppose we define deficiencies $D_{N}{ }^{\prime}(\xi)$ by

$$
P_{F, \mu}\left(\left|M_{N+D_{N}^{\prime}}-\mu\right| \leqq \xi N^{-\frac{1}{2}}\right)=P_{F, \mu, \Delta}\left(\left|M_{\Delta, N}^{\prime}-\mu\right| \leqq \xi N^{-\frac{1}{2}}\right)
$$

for that value of $\Delta$ that minimizes the right-hand side. In view of remark (ii), Theorem 8.3 implies that $D_{N}{ }^{\prime}(\xi)$ is also asymptotically equivalent to the $\tilde{D}_{N, i}$. Thus our results can be thought of as corresponding exactly to those of ABZ (1976) where deficiencies are defined in terms of a positive quantile of the symmetrically distributed centered estimators in the one-sample problem. Since the deficiency is asymptotically independent of the value of $\xi$, we obtain the same answers for deficiencies based on reasonable functionals of the distributions of $N^{\frac{1}{2}}|M-\mu|$ and $N^{\frac{1}{2}}\left|M^{\prime}-\mu\right|$, such as the asymptotic second moment. This agrees with what was found in the one-sample case in Albers (1974).

The choice $\Delta=\lambda$ is less obvious in equation (8.20) which defines $D_{N}(\xi)$. In remark (iii) we pointed out that if we would not choose $\Delta=\lambda+o\left(N^{-\frac{1}{2}}\right)$, then our results would become essentially more complicated. The first source of trouble is the difference of the significance levels $\bar{\alpha}$ and $\tilde{\alpha}$ given by (8.2) and (8.15). Except in the trivial case where $\int \Psi_{1}{ }^{3}(t) d t=0$, we find that $(\tilde{\alpha}-\bar{\alpha})$ is of the order of $N^{-\frac{1}{2}}(\Delta-\lambda)$ and a change of the order of $N^{-\frac{1}{2}}(\Delta-\lambda)$ in the level of significance of one of the two tests produces a change of the same order in its power. Unless $\Delta-\lambda=o\left(N^{-\frac{1}{2}}\right)$ such an effect is not negligible for our purposes and this means that it would no longer be true that the deficiency for the estimators is asymptotically equivalent in the sense of (8.22) to the deficiency of the parent tests at the same level. In fact a correction term of the order of $N^{\frac{1}{2}}(\Delta-\lambda)$ would have to be introduced in (8.22) to ensure its validity. Note that there is no contradiction here with the fact that in the proof of Theorem 8.3 we could change $\bar{\alpha}$ to $\frac{1}{2}$ with impunity, because there we were concerned with the same change of level for both tests simultaneously. A second unpleasant consequence of choosing $\Delta=\Delta^{0}$ (or even $\Delta=\lambda+b N^{-\frac{1}{2}}$ with $b$ independent of $x$ ) would be that the expansions for $D_{N}(\xi)$ would no longer be independent of 
$\xi$. By taking $\Delta=\Delta^{0}$, we would therefore destroy at one stroke the two most striking features of Theorem 8.3.

Next we note that upon formal substitution of $\alpha=\frac{1}{2}$ and $\theta=0$ the expansions for $d_{N}$ in Theorem 7.1 reduce to the expansions for $D_{N}(\xi)$ in Theorem 8.3. This shows that for every $\xi \neq 0, D_{N}(\xi)$ is nonnegative for sufficiently large $N$.

In the proof of Theorem 8.3 we indicated how one can obtain expansions for the deficiency of the locally most powerful rank test or its approximate scores analogue with respect to the locally most powerful test. At that point there was no need to produce these expansions, but we shall do so now because they may be of independent interest. The simplest way to describe these results is the following. In the formulation of Theorem 7.1 change the words "most powerful test" to "locally most powerful test" and $K_{F, \theta, \Delta_{0}}$ to $K_{F, \theta, \lambda}$; change $\tilde{d}_{N, 0}$ in $(7.8)$ to

$$
\tilde{d}_{N, 0}=\frac{1}{4 \overline{8}}\left[\frac{4 \int \Psi_{1}^{4}(t) d t}{\left\{\int \Psi_{1}^{2}(t) d t\right\}^{2}}\left\{3\left(u_{\alpha}^{2}-1\right)-2 \eta^{*} u_{\alpha}\right\}-12\left(u_{\alpha}^{2}+3-2 \eta^{*} u_{\alpha}\right)\right] .
$$

With these changes Theorem 7.1 holds. When comparing the expansions for $d_{N}$ in (7.8) with those based on (8.28) we see that the expansions in (7.8) consist of three parts. The term involving $\left(2 u_{\alpha}-\eta^{*}\right)^{2}$ is due to the fact that comparisons with the most powerful test were made for $\Delta=\Delta_{0}$ rather than $\Delta=\lambda$ (cf. the discussion following Theorem 7.1). The other terms involving $\eta^{* 2}$ represent the deficiency of the locally most powerful test with respect to the most powerful test for $\Delta=\lambda$. The remaining terms are due to the transition from the locally most powerful test to the two rank tests. All four tests are efficient to second order, i.e., for each pair the deficiency is $o\left(N^{\frac{1}{2}}\right)$, and the reason for this is that the terms of orders 1 and $N^{-\frac{1}{2}}$ are the same in all four power expansions (cf. the discussion following Theorem 7.1).

We conclude with one example of Theorem 8.3. For estimating $\mu$ in the normal location model $(\Phi(\cdot+\lambda \mu), \Phi(\cdot-(1-\lambda) \mu))$, the deficiency of either one of the Hodges-Lehmann estimators associated with the normal scores test and with van der Waerden's test with respect to the difference of the sample means is given by

$$
D_{N}(\xi)=\log \log N-\frac{3}{2}+\log 2+\gamma+o(1),
$$

where $\gamma$ is Euler's constant as in (5.50). Note that this expansion is the same as expansion (7.23) for the deficiency of the normal scores test (or van der Waerden's test) with respect to Student's test for any $\alpha$.

\section{APPENDIX}

Expansions for the contiguous location case. In this appendix we provide the tools for deriving Theorem 4.1 from Theorem 3.1. The quantities appearing in the expansion of Theorem 3.1 are expected values under $P_{H}$ of functions of $P_{1}, \ldots, P_{N}$ and in the setup of Section 4 both $H$ and $P_{1}, \ldots, P_{N}$ depend on $\theta$. 
Our task is to provide Taylor expansions in $\theta$ with error bounds for these quantities, thus reducing expectations $E_{H}$ to expectations $E_{F}$ while at the same time expanding the rv's involved. Since we are only concerned with the models $P_{H}$ and $P_{F}$ under the assumptions of Section 4, we suppose throughout that $X_{1}, \cdots, X_{N}$ are i.i.d. with common density $h$ under $P_{H}$ and $f$ under $P_{F}$, where $h(x)=(1-\lambda) f(x)+\lambda f(x-\theta)$ and $f$ is positive and four times differentiable on $R^{1}$. Define $\xi(x, t), p(x, t)$ and $\tilde{p}(x, t)$ by

$$
(1-\lambda) F(\xi(x, t))+\lambda F(\xi(x, t)-t)=F(x),
$$

$$
p(x, t)=\frac{\lambda f(x-t)}{(1-\lambda) f(x)+\lambda f(x-t)} .
$$

$$
\tilde{p}(x, t)=p(\xi(x, t), t) \text {. }
$$

As in Appendix 1 of ABZ (1976), these functions are introduced because $\tilde{p}\left(Z_{1}, \theta\right), \cdots, \tilde{p}\left(Z_{N}, \theta\right)$ under $P_{F}$ have the same joint distribution as $P_{1}, \ldots, P_{N}$ under $P_{H}$. Our main problem is therefore to expand $\tilde{p}(x, t)$ as a function of $t$ around $t=0$.

With $\phi_{i}=f^{(i)} / f$ as in (4.1), we define for $i=1, \ldots, 4$,

$$
\chi_{i}(x, t)=\left|\psi_{i}(\xi(x, t))\right|+\left|\psi_{i}(\xi(x, t)-t)\right|
$$

and for any function $q$ of two variables we write

$$
q_{i, j}(x, t)=\frac{\partial^{i+j} q(x, t)}{\partial x^{i} \partial t^{j}}
$$

Then elementary but tedious computations yield

$$
\begin{aligned}
& \tilde{p}(x, 0)= \lambda \\
& \tilde{p}_{0,1}(x, 0)=-\lambda(1-\lambda) \psi_{1}(x) \\
& \tilde{p}_{0,2}(x, 0)=\lambda(1-\lambda)(1-2 \lambda) \psi_{2}(x) \\
& \tilde{p}_{0,3}(x, 0)=-\lambda(1-\lambda)\left\{\left(1-3 \lambda+3 \lambda^{2}\right) \psi_{3}(x)-6 \lambda(1-\lambda) \psi_{1}(x) \psi_{2}(x)\right. \\
&\left.+3 \lambda(1-\lambda) \psi_{1}^{3}(x)\right\} \\
& \\
&\left|\tilde{p}_{0,1}\right| \leqq b_{1} \chi_{1} \\
&\left|\tilde{p}_{0,2}\right| \leqq b_{2}\left(\chi_{2}+\chi_{1}^{2}\right) \\
&\left|\tilde{p}_{0,3}\right| \leqq b_{3}\left(\chi_{3}+\chi_{2}^{\frac{3}{2}}+\chi_{1}^{3}\right) \\
&\left|\tilde{p}_{0,4}\right| \leqq b_{4}\left(\chi_{4}+\chi_{3}^{3}+\chi_{2}^{2}+\chi_{1}^{4}\right)
\end{aligned}
$$

where $b_{1}, \cdots, b_{4}$ are positive constants.

Define $\pi_{j}=E_{H} P_{j}$ as in (3.16).

THEOREM A.1. Suppose that positive numbers $C, C^{\prime}$ and $\varepsilon^{\prime}$ exist such that $\sum a_{j}{ }^{4} \leqq C N, 0 \leqq \theta \leqq \varepsilon^{\prime}$ and (4.2) is satisfied. Then there exists $B>0$ depending 
only on $C, C^{\prime}$ and $\varepsilon^{\prime}$ such that

(A.7)

$$
\begin{aligned}
& \sum a_{j}\left(\pi_{j}-\lambda\right)=\lambda(1-\lambda)\left\{-\theta \sum a_{j} E_{F} \psi_{1}\left(Z_{j}\right)\right. \\
& +(1-2 \lambda) \frac{\theta^{2}}{2} \sum a_{j} E_{F} \psi_{2}\left(Z_{j}\right) \\
& -\frac{\theta^{3}}{6} \sum a_{j} E_{F}\left[\left(1-3 \lambda+3 \lambda^{2}\right) \psi_{3}\left(Z_{j}\right)\right. \\
& \left.\left.-6 \lambda(1-\lambda) \phi_{1}\left(Z_{j}\right) \phi_{2}\left(Z_{j}\right)+3 \lambda(1-\lambda) \dot{\varphi}_{1}{ }^{3}\left(Z_{j}\right)\right]\right\}+M_{1}, \\
& \left|M_{1}\right| \leqq B N^{\frac{5}{4}} \theta^{4} \\
& \sum a_{j}{ }^{2}\left(\pi_{j}-\lambda\right)=\lambda(1-\lambda)\left\{-\theta \sum a_{j}{ }^{2} E_{F} \psi_{1}\left(Z_{j}\right)\right. \\
& \left.+(1-2 \lambda) \frac{\theta^{2}}{2} \sum a_{j}^{2} E_{F} \psi_{2}\left(Z_{j}\right)\right\}+M_{2}, \\
& \left|M_{2}\right| \leqq B N^{\frac{5}{4}} \theta^{3} \\
& \text { (A.9) } \quad \sum a_{j}{ }^{3}\left(\pi_{j}-\lambda\right)=-\lambda(1-\lambda) \theta \sum a_{j}{ }^{3} E_{F} \psi_{1}\left(Z_{j}\right)+M_{3} \text {, } \\
& \left|M_{3}\right| \leqq B N^{\frac{13}{2}} \theta^{2} \text {; } \\
& \text { (A.10) } \quad \sum a_{j}{ }^{2} E_{H}\left(P_{j}-\lambda\right)^{2}=\lambda^{2}(1-\lambda)^{2} \theta^{2} \sum a_{j}{ }^{2} E_{F} \phi_{1}{ }^{2}\left(Z_{j}\right)+M_{4} \text {, } \\
& \left|M_{4}\right| \leqq B N^{\frac{5}{4}} \theta^{3} \text {; } \\
& \sigma_{H}^{2}\left(\sum a_{j} P_{j}\right)=\lambda^{2}(1-\lambda)^{2} \theta^{2} \sigma_{F}{ }^{2}\left(\sum a_{j} \psi_{1}\left(Z_{j}\right)\right)+M_{5},
\end{aligned}
$$

(A.11) $\quad\left|M_{5}\right| \leqq B\left\{N^{2} \theta^{24}+N \theta^{19}\left[E_{F}\left|\sum a_{j}\left(\phi_{1}\left(Z_{j}\right)-E_{F} \psi_{1}\left(Z_{j}\right)\right)\right|^{3}\right]^{\frac{1}{3}}\right.$

$$
\left.+\theta^{3} \sigma_{F}\left(\sum a_{j} \psi_{1}\left(Z_{j}\right)\right) \sigma_{F}\left(\sum a_{j} \psi_{2}\left(Z_{j}\right)\right)+\theta^{4} \sigma_{F}{ }^{2}\left(\sum a_{j} \psi_{2}\left(Z_{j}\right)\right)\right\}
$$

$$
E_{H}\left(\frac{\lambda g\left(X_{1}\right)}{h\left(X_{1}\right)}-\lambda\right)^{4} \leqq B \theta^{4}
$$

(A.13) $\quad\left[\sum\left\{E_{H}\left|P_{j}-\pi_{j}\right|^{3}\right\}^{\frac{9}{9}}\right]^{\frac{9}{4}} \leqq \theta^{3}\left[\sum\left\{E_{F} \mid \psi_{1}\left(Z_{j}\right)-E_{F} \psi_{1}\left(Z_{j}\right)^{\mid 3}\right\}^{\frac{9}{9}}\right]^{\frac{9}{4}}+B N^{\frac{9}{4}} \theta^{6}$.

Proof. Although the proof is very similar to that of Theorem A1.1 and the relevant part of Corollary A1.1 in ABZ (1976), there are additional complications due to the fact that now $\tilde{p}_{0,2}(x, 0) \not \equiv 0$. We begin by noting that the distribution of $\xi\left(X_{1}, t\right)$ under $F$ is that of $X_{1}$ under $\lambda F(x)+(1-\lambda) F(x-t)$, so that (4.2) and (A.6) imply the existence of $B_{1}>0$ depending only on $C^{\prime}$ and such that

$$
\sup \left\{E_{F}\left|\tilde{p}_{0, i}\left(X_{1}, \nu \theta\right)\right|^{m_{i}}: 0 \leqq \nu \leqq 1\right\} \leqq B_{1}, \quad i=1, \cdots, 4,
$$

where $m_{1}=6, m_{2}=3, m_{3}=\frac{4}{3}, m_{4}=1$.

Using Lemma A1.1 of ABZ (1976) together with $\sum a_{j}{ }^{4} \leqq C N$ and (A.14), we find that

$$
\begin{aligned}
\left|M_{1}\right| & \leqq \frac{\theta^{4}}{24} \sup \left\{\sum\left|a_{j}\right| E_{F}\left|\tilde{p}_{0,4}\left(Z_{j}, \nu \theta\right)\right|: 0 \leqq \nu \leqq 1\right\} \\
& \leqq \frac{(C N)^{\frac{1}{4}} \theta^{4}}{24} \sup \left\{N E_{F}\left|\tilde{p}_{0,4}\left(X_{1}, \nu \theta\right)\right|: 0 \leqq \nu \leqq 1\right\} \leqq \frac{B_{1} C^{\frac{1}{4}}}{24} N^{\frac{5}{4}} \theta^{4},
\end{aligned}
$$




$$
\begin{aligned}
& \left|M_{2}\right| \leqq \frac{\theta^{3}}{6} \sup \left\{\sum a_{j}{ }^{2} E_{F}\left|\tilde{p}_{0,3}\left(Z_{j}, \nu \theta\right)\right|: 0 \leqq \nu \leqq 1\right\} \\
& \leqq \frac{\theta^{3}}{6}\left(\sum a_{j}{ }^{8}\right)^{\frac{1}{4}} \sup \left\{\left[N E_{F}\left|\tilde{p}_{0,3}\left(X_{1}, \nu \theta\right)\right|^{\frac{9}{3}}\right]^{\frac{3}{4}}: 0 \leqq \nu \leqq 1\right\} \leqq \frac{B_{1}{ }^{\frac{3}{4}} C^{\frac{1}{2}}}{6} N^{\frac{5}{2}} \theta^{3} \text {, } \\
& \left|M_{3}\right| \leqq \frac{\theta^{2}}{2} \sup \left\{\sum\left|a_{j}\right|^{3} E_{F}\left|\tilde{p}_{0,2}\left(Z_{j}, \nu \theta\right)\right|: 0 \leqq \nu \leqq 1\right\} \\
& \leqq \frac{\theta^{2}}{2}\left(\sum\left|a_{j}\right|^{\frac{9}{2}}\right)^{\frac{2}{3}}\left(N B_{1}\right)^{\frac{1}{3}} \leqq \frac{B_{1}^{\frac{1}{3}} C^{\frac{3}{4}}}{2} N^{\frac{13}{12}} \theta^{2}, \\
& \left|M_{4}\right| \leqq \frac{\theta^{3}}{6} \sup \left\{\sum a_{j}{ }^{2} E_{F}\left[2\left|\tilde{p}_{0,3}\left(Z_{j}, \nu \theta\right)\right|+6\left|\tilde{p}_{0,1}\left(Z_{j}, \nu \theta\right) \tilde{p}_{0,2}\left(Z_{j}, \nu \theta\right)\right|\right]: 0 \leqq \nu \leqq 1\right\} \\
& \leqq \frac{\theta^{3}}{6}\left[2\left(\sum a_{j}\right)^{\frac{1}{4}}\left(N B_{1}\right)^{\frac{3}{4}}+6\left(\sum a_{j}\right)^{\frac{1}{2}}\left(N B_{1}\right)^{\frac{1}{2}}\right] \leqq\left(B_{1}^{\frac{1}{2}}+B_{1^{\frac{3}{4}}}\right) C^{\frac{1}{2}} N^{\frac{5}{2}} \theta^{3}, \\
& E_{H}\left(\frac{\lambda g\left(X_{1}\right)}{h\left(X_{1}\right)}-\lambda\right)^{4} \leqq \theta^{4} \sup \left\{E_{F} \tilde{p}_{0,1}^{4}\left(X_{1}, \nu \theta\right): 0 \leqq \nu \leqq 1\right\} \leqq B_{1}^{\frac{2}{3}} \theta^{4},
\end{aligned}
$$

which proves (A.7)-(A.10) and (A.12). To establish (A.13) we note that

$$
\begin{aligned}
\left|\tilde{p}\left(Z_{j}, \theta\right)-E_{F} \tilde{p}\left(Z_{j}, \theta\right)\right| \leqq & \theta\left|\tilde{p}_{0,1}\left(Z_{j}, 0\right)-E_{F} \tilde{p}_{0,1}\left(Z_{j}, 0\right)\right| \\
& +\frac{\theta^{2}}{2} \int_{0}^{1} 2(1-\nu)\left\{\left|\tilde{p}_{0,2}\left(Z_{j}, \nu \theta\right)\right|+E_{F}\left|\tilde{p}_{0,2}\left(Z_{j}, \nu \theta\right)\right|\right\} d \nu .
\end{aligned}
$$

Hence

$$
\begin{gathered}
E_{H}\left|P_{j}-\pi_{j}\right|^{3} \leqq \frac{\theta^{3}}{16} E_{F}\left|\psi_{1}\left(Z_{j}\right)-E_{F} \psi_{1}\left(Z_{j}\right)\right|^{3}+4 \theta^{6} \int_{0}^{1} 2(1-\nu) E_{F}\left|\tilde{p}_{0,2}\left(Z_{j}, \nu \theta\right)\right|^{3} d \nu, \\
\sum\left\{E_{H}\left|P_{j}-\pi_{j}\right|^{3}\right\}^{\frac{4}{5}} \leqq \theta^{3} \sum\left\{E_{F}\left|\psi_{1}\left(Z_{j}\right)-E_{F} \psi_{1}\left(Z_{j}\right)\right|^{3}\right\}^{\frac{4}{3}}+2\left(B_{1}+1\right) N \theta^{\frac{8}{3}},
\end{gathered}
$$

and (A.13) follows.

It remains to prove (A.11). We have

$$
\begin{aligned}
\tilde{p}(x, t) & -\lambda+\lambda(1-\lambda) t \psi_{1}(x)-\frac{1}{2} \lambda(1-\lambda)(1-2 \lambda) t^{2} \psi_{2}(x) \\
= & \frac{t^{2}}{2} \int_{0}^{1} 2(1-\nu)\left(\tilde{p}_{0,2}(x, \nu t)-\tilde{p}_{0,2}(x, 0)\right) d \nu=\frac{t^{3}}{6} \int_{0}^{1} 3(1-\nu)^{2} \tilde{p}_{0,3}(x, \nu t) d \nu,
\end{aligned}
$$

and as a result

$$
\begin{aligned}
&\left(\tilde{p}(x, t)-\lambda+\lambda(1-\lambda) t \psi_{1}(x)-\frac{1}{2} \lambda(1-\lambda)(1-2 \lambda) t^{2} \psi_{2}(x)\right)^{2} \\
& \leqq\left|\frac{t^{2}}{2} \int_{0}^{1} 2(1-\nu)\left(\tilde{p}_{0,2}(x, \nu t)-\tilde{p}_{0,2}(x, 0)\right) d \nu\right|^{\frac{6}{3}}\left|\frac{t^{3}}{6} \int_{0}^{1} 3(1-\nu)^{2} \tilde{p}_{0,3}(x, \nu t) d \nu\right|^{\frac{4}{3}} \\
& \leqq|t|^{24}\left\{\left|\frac{1}{2} \int_{0}^{1} 2(1-\nu)\left(\tilde{p}_{0,2}(x, \nu t)-\tilde{p}_{0,2}(x, 0)\right) d \nu\right|^{3}\right. \\
&\left.\quad \quad+\left|\frac{1}{6} \int_{0}^{1} 3(1-\nu)^{2} \tilde{p}_{0,3}(x, \nu t) d \nu\right|^{4}\right\} \\
& \leqq|t|^{24} \int_{0}^{1}\left\{\left|\tilde{p}_{0,2}(x, \nu t)\right|^{3}+\left|\tilde{p}_{0,2}(x, 0)\right|^{3}+\left|\tilde{p}_{0,3}(x, \nu t)\right|^{3}\right\} d \nu .
\end{aligned}
$$

Similarly,

$$
\begin{aligned}
\mid \tilde{p}(x, t)-\lambda+ & \lambda(1-\lambda) t \phi_{1}(x)-\left.\frac{1}{2} \lambda(1-\lambda)(1-2 \lambda) t^{2} \phi_{2}(x)\right|^{\frac{3}{2}} \\
& \leqq|t|^{\frac{2}{5}} \int_{0}^{1}\left\{\left|\tilde{p}_{0,2}(x, \nu t)\right|^{3}+\left|\tilde{p}_{0,2}(x, 0)\right|^{3}+\left|\tilde{p}_{0,3}(x, \nu t)\right|^{4}\right\} d \nu .
\end{aligned}
$$


It follows that

$$
\begin{aligned}
& \sigma_{F}^{2}\left(\sum a_{j}\left\{\tilde{p}\left(Z_{j}, \theta\right)+\lambda(1-\lambda) \theta \psi_{1}\left(Z_{j}\right)-\frac{1}{2} \lambda(1-\lambda)(1-2 \lambda) \theta^{2} \psi_{2}\left(Z_{j}\right)\right\}\right) \\
& \leqq N \sum a_{j}{ }^{2} E_{F}\left(\tilde{p}\left(X_{1}, \theta\right)-\lambda+\lambda(1-\lambda) \theta \psi_{1}\left(X_{1}\right)-\frac{1}{2} \lambda(1-\lambda)(1-2 \lambda) \theta^{2} \psi_{2}\left(X_{1}\right)\right)^{2} \\
& \leqq 3 B_{1} C^{\frac{1}{2}} N^{2} \theta^{24} \text {, } \\
& \mid \operatorname{Cov}_{F}\left(\sum a _ { j } \left\{\tilde{p}\left(Z_{j}, \theta\right)+\lambda(1-\lambda) \theta \psi_{1}\left(Z_{j}\right)\right.\right. \\
& \left.\left.-\frac{1}{2} \lambda(1-\lambda)(1-2 \lambda) \theta^{2} \psi_{2}\left(Z_{j}\right)\right\}, \sum a_{j} \psi_{1}\left(Z_{j}\right)\right) \\
& \leqq\left[E_{F} \mid \sum a_{j}\left\{\tilde{p}\left(Z_{j}, \theta\right)-\lambda+\lambda(1-\lambda) \theta \psi_{1}\left(Z_{j}\right)\right.\right. \\
& \left.\left.-\frac{1}{2} \lambda(1-\lambda)(1-2 \lambda) \theta^{2} \psi_{2}\left(Z_{j}\right)\right\}\left.\right|^{\frac{3}{2}}\right]^{\frac{2}{3}}\left[E_{F}\left|\sum a_{j}\left(\psi_{1}\left(Z_{j}\right)-E_{F} \psi_{1}\left(Z_{j}\right)\right)\right|^{3}\right]^{\frac{1}{3}} \\
& \leqq\left[\left(\sum\left|a_{j}{ }^{3}\right|\right)^{\frac{1}{2}} N E_{F} \mid \tilde{p}\left(X_{1}, \theta\right)-\lambda+\lambda(1-\lambda) \theta \psi_{1}\left(X_{1}\right)\right. \\
& \left.-\left.\frac{1}{2} \lambda(1-\lambda)(1-2 \lambda) \theta^{2} \psi_{2}\left(X_{1}\right)\right|^{\frac{8}{2}}\right]^{\frac{2}{3}}\left[E_{F}\left|\sum a_{j}\left(\psi_{1}\left(Z_{j}\right)-E_{F} \psi_{1}\left(Z_{j}\right)\right)\right|^{3}\right]^{\frac{1}{3}} \\
& \leqq\left(3 B_{1}\right)^{\frac{2}{3}} C^{\frac{1}{4}} N \theta^{\frac{14}{5}}\left[E_{F}\left|\sum a_{j}\left(\phi_{1}\left(Z_{j}\right)-E_{F} \psi_{1}\left(Z_{j}\right)\right)\right|^{3}\right]^{\frac{1}{3}} \text {, } \\
& \mid \operatorname{Cov}_{F}\left(\sum a _ { j } \left\{\tilde{p}\left(Z_{j}, \theta\right)+\lambda(1-\lambda) \theta \phi_{1}\left(Z_{j}\right)\right.\right. \\
& \left.\left.-\frac{1}{2} \lambda(1-\lambda)(1-2 \lambda) \theta^{2} \psi_{2}\left(Z_{j}\right)\right\}, \sum a_{j} \psi_{2}\left(Z_{j}\right)\right) \mid \\
& \leqq\left(3 B_{1}\right)^{\frac{1}{2}} C^{\frac{1}{4}} N \theta^{\frac{12}{5}} \sigma_{F}\left(\sum a_{j} \psi_{2}\left(Z_{j}\right)\right) \text {. }
\end{aligned}
$$

These inequalities ensure that there exists $B_{2}>0$ depending only on $B_{1}$ and $C$ such that

$$
\begin{aligned}
& \left|\sigma_{H}^{2}\left(\sum a_{j} P_{j}\right)-\sigma_{F}^{2}\left(\sum a_{j}\left\{\lambda(1-\lambda) \theta \phi_{1}\left(Z_{j}\right)-\frac{1}{2} \lambda(1-\lambda)(1-2 \lambda) \theta^{2} \psi_{2}\left(Z_{j}\right)\right\}\right)\right| \\
& \quad \leqq B_{2}\left\{N^{2} \theta^{24}+N \theta^{\frac{19}{5}}\left[E_{F}\left|\sum a_{j}\left(\phi_{1}\left(Z_{j}\right)-E_{F} \psi_{1}\left(Z_{j}\right)\right)\right|^{3}\right]^{\frac{1}{3}}+N \theta^{2 \frac{22}{5}} \sigma_{F}\left(\sum a_{j} \psi_{2}\left(Z_{j}\right)\right)\right\} .
\end{aligned}
$$

Since $N \theta^{23} \sigma_{F}\left(\sum a_{j} \psi_{2}\left(Z_{j}\right)\right) \leqq N^{2} \theta^{24}+\theta^{4}{\sigma_{F}}^{2}\left(\sum a_{j} \psi_{2}\left(Z_{j}\right)\right)$, (A.11) follows immediately and the proof of the theorem is complete.

Corollary A.1. Suppose that (3.1) and (4.3) hold and that positive numbers $c, C, C^{\prime}, D$, $\varepsilon$ and $\varepsilon^{\prime}$ exist such that (3.10), (3.19), (4.2) and (4.4) are satisfied. Let $K, \alpha_{i}, \tilde{K}, \tilde{\alpha}_{i}$ and $\eta$ be defined by (3.17), (3.18), (4.5), (4.6) and (4.7). Then there exists $B>0$ depending only on $c, C, C^{\prime}, D$, $\varepsilon$ and $\varepsilon^{\prime}$ such that

$$
\begin{gathered}
\sup _{x}\left|K\left(x-\frac{\sum a_{j} \pi_{j}}{\left\{\lambda(1-\lambda) \sum a_{j}{ }^{2}\right\}^{\frac{1}{2}}}\right)-\tilde{K}(x-\eta)\right| \\
\leqq B\left\{N^{-\frac{5}{4}}+N^{-\frac{1}{2}} \theta^{3}\left[\sum\left\{E_{F}\left|\psi_{1}\left(Z_{j}\right)-E_{F} \psi_{1}\left(Z_{j}\right)\right|^{3}\right\}^{\frac{9}{3}}\right]^{\frac{9}{4}}\right. \\
\left.+N^{-\frac{3}{4}} \theta^{3}\left[\sum\left\{E_{F}\left(\psi_{2}\left(Z_{j}\right)-E_{F} \psi_{2}\left(Z_{j}\right)\right)^{2}\right\}^{\frac{2}{3}}\right]^{\frac{3}{2}}\right\}
\end{gathered}
$$

(A.16) $\quad \theta^{2} \frac{\left|\sum a_{j} E_{F} \psi_{2}\left(Z_{j}\right)\right|}{\left(\sum a_{j}{ }^{2}\right)^{\frac{1}{2}}} \leqq B N^{-\frac{1}{2}}, \quad \theta \frac{\left|\sum a_{j}{ }^{2} E_{F} \psi_{1}\left(Z_{j}\right)\right|}{\sum a_{j}{ }^{2}} \leqq B N^{-\frac{1}{2}}$,

$$
\frac{\left|\sum a_{j}{ }^{3}\right|}{\left(\sum a_{j}{ }^{2}\right)^{\frac{3}{2}}} \leqq B N^{-\frac{1}{2}} \text {, }
$$

(A. 17) $\quad \theta^{2} \frac{\sigma_{F}{ }^{2}\left(\sum a_{j} \psi_{1}\left(Z_{j}\right)\right)}{\sum a_{j}{ }^{2}} \leqq B\left\{N^{-1}+N^{-\frac{1}{4}} \theta^{3}\left[\sum\left\{E_{F}\left|\psi_{1}\left(Z_{j}\right)-E_{F} \psi_{1}\left(Z_{j}\right)\right|^{3}\right\}^{\frac{9}{3}}\right]^{\frac{9}{4}}\right\}$

and all other terms occurring in $\tilde{\alpha}_{0}, \ldots, \tilde{\alpha}_{5}$ are bounded in absolute value by $B N^{-1}$. 
Proof. In this proof $O(x)$ will denote a quantity that is bounded by $B_{1}|x|$ with $B_{1}$ depending only on $c, C, C^{\prime}, D, \varepsilon$ and $\varepsilon^{\prime}$.

We begin by noting that (A.16) and the last statement in Corollary A.1 are immediate consequences of Hölder's inequality, (3.10), (4.2) and (4.4). Also

$$
\begin{aligned}
\theta^{2} \sigma_{F}{ }^{2}\left(\sum a_{j} \psi_{1}\left(Z_{j}\right)\right) & \leqq 1+\theta^{3} \sigma_{F}{ }^{3}\left(\sum a_{j} \psi_{1}\left(Z_{j}\right)\right) \\
& \leqq 1+\theta^{3} E_{F}\left|\sum a_{j}\left(\psi_{1}\left(Z_{j}\right)-E_{F} \psi_{1}\left(Z_{j}\right)\right)\right|^{3} \\
& \leqq 1+\theta^{3}\left[\sum\left|a_{j}\right|\left\{E_{F}\left|\psi_{1}\left(Z_{j}\right)-E_{F} \psi_{1}\left(Z_{j}\right)\right|^{3}\right\}^{3}\right]^{3} \\
& \leqq 1+\theta^{3}\left(\sum a_{j}^{4}\right)^{\frac{3}{2}}\left[\sum\left\{E_{F}\left|\psi_{1}\left(Z_{j}\right)-E_{F} \psi_{1}\left(Z_{j}\right)\right|^{3}\right\}^{3}\right]^{\frac{9}{4}}
\end{aligned}
$$

and in view of (3.10) and (4.4), this implies (A.17). For later use we note that similarly

$$
\sigma_{F}^{2}\left(\sum a_{j} \psi_{2}\left(Z_{j}\right)\right) \leqq C^{\frac{1}{2}} N^{\frac{1}{2}}\left[\sum\left\{E_{F}\left(\psi_{2}\left(Z_{j}\right)-E_{F} \psi_{2}\left(Z_{j}\right)\right)^{2}\right\}^{\frac{2}{3}}\right]^{\frac{3}{2}} .
$$

It remains to prove (A.15). Since (A.15) is trivially satisfied for $N<\left(D / \varepsilon^{\prime}\right)^{2}$, we may assume that $0 \leqq \theta \leqq \varepsilon^{\prime}$ so that Theorem A.1 applies. Because of (3.1), $\sum a_{j} \pi_{j}=\sum a_{j}\left(\pi_{j}-\lambda\right)$. In view of the bounds obtained above, we can truncate expansions (A.7) and (A.8) to

$$
\begin{aligned}
\sum a_{j} \pi_{j}= & \lambda(1-\lambda)\left\{-\theta \sum a_{j} E_{F} \psi_{1}\left(Z_{j}\right)\right. \\
& \left.+(1-2 \lambda) \frac{\theta^{2}}{2} \sum a_{j} E_{F} \psi_{2}\left(Z_{j}\right)\right\}+O\left(N \theta^{3}\right) \\
= & -\lambda(1-\lambda) \theta \sum a_{j} E_{F} \psi_{1}\left(Z_{j}\right)+O\left(N \theta^{2}\right)=O(N \theta),
\end{aligned}
$$

(A.21) $\quad \sum a_{j}{ }^{2}\left(\pi_{j}-\lambda\right)=-\lambda(1-\lambda) \theta \sum a_{j}{ }^{2} E_{F} \psi_{1}\left(Z_{j}\right)+O(1)=O\left(N^{\frac{1}{2}}\right)$.

Using (A.8)-(A.11), (A.20), (A.21), (3.10), (3.19) and (4.4) we expand $\alpha_{0}, \cdots, \alpha_{5}$ and find

$$
\begin{aligned}
\sup _{x}|K(x)-\hat{K}(x)|=O & \left(N^{-\frac{8}{4}}+\theta^{19}\left[E_{F}\left|\sum a_{j}\left(\psi_{1}\left(Z_{j}\right)-E_{F} \psi_{1}\left(Z_{j}\right)\right)\right|^{3}\right]^{\frac{1}{3}}\right. \\
& +N^{-1} \theta^{3} \sigma_{F}\left(\sum a_{j} \psi_{1}\left(Z_{j}\right)\right) \sigma_{F}\left(\sum a_{j} \psi_{2}\left(Z_{j}\right)\right) \\
& \left.+N^{-1} \theta^{4} \sigma_{F}^{2}\left(\sum a_{j} \psi_{2}\left(Z_{j}\right)\right)\right)
\end{aligned}
$$

where

$$
\begin{gathered}
\hat{K}(x)=\Phi(x)-\phi(x) \sum_{k=0}^{5} \hat{\alpha}_{k} H_{k}(x), \\
\hat{\alpha}_{0}=-\left(\frac{\lambda(1-\lambda)}{\sum a_{j}{ }^{2}}\right)^{\frac{1}{2}} N^{-1} \theta \sum a_{j} E_{F} \psi_{1}\left(Z_{j}\right), \\
\hat{\alpha}_{1}=\tilde{\alpha}_{1}-\frac{1}{8 \sum a_{j}^{2}} \lambda(1-\lambda)(1-2 \lambda)^{2} \theta^{4}\left\{\sum a_{j} E_{F} \psi_{2}\left(Z_{j}\right)\right\}^{2}, \\
(\mathrm{~A} .24) \quad \hat{\alpha}_{2}=\tilde{\alpha}_{2}-\frac{\{\lambda(1-\lambda)\}^{\frac{1}{2}}}{4\left(\sum a_{j}{ }^{2}\right)^{\frac{3}{2}}}(1-2 \lambda)^{2} \theta^{3} \sum a_{j}{ }^{2} E_{F} \psi_{1}\left(Z_{j}\right) \sum a_{j} E_{F} \psi_{2}\left(Z_{j}\right), \\
\hat{\alpha}_{3}=\tilde{\alpha}_{3}-\frac{1}{12\left(\sum a_{j}{ }^{2}\right)^{2}}(1-2 \lambda)^{2} \theta^{2} \sum a_{j}{ }^{3} \sum a_{j} E_{F} \psi_{2}\left(Z_{j}\right), \\
\hat{\alpha}_{k}=\tilde{\alpha}_{k} \quad \text { for } k=4,5,
\end{gathered}
$$


with $\tilde{\alpha}_{k}$ as given by (4.6). By applying elementary inequalities (A.22) may be simplified to

$$
\begin{aligned}
\text { (A.25) } \sup _{x}|K(x)-\hat{K}(x)|=O & \left(N^{-\frac{5}{4}}+N^{-\frac{5}{4}} \theta^{3} E_{F}\left|\sum a_{j}\left(\phi_{1}\left(Z_{j}\right)-E_{F} \psi_{1}\left(Z_{j}\right)\right)\right|^{3}\right. \\
& \left.+N^{-\frac{5}{4}} \theta^{3} \sigma_{F}^{2}\left(\sum a_{j} \psi_{2}\left(Z_{j}\right)\right)\right) .
\end{aligned}
$$

With the aid of (A.7), (A.20) and the bounds obtained in the first part of the proof we now expand $\hat{K}\left(x-\sum a_{j} \pi_{j}\left\{\lambda(1-\lambda) \sum a_{j}{ }^{2}\right\}^{-\frac{1}{2}}\right)$ about the point $(x-\eta)$ and obtain

$$
\begin{array}{r}
\sup _{x} \mid \hat{K}\left(x-\frac{\sum a_{j} \pi_{j}}{\left\{\lambda(1-\lambda) \sum a_{j}^{2}\right\}^{\frac{1}{2}}}-\tilde{K}(x-\eta) \mid\right. \\
=O\left(N^{-\frac{5}{4}}+N^{-1} \theta^{3}{\sigma_{F}}^{2}\left(\sum a_{j} \psi_{1}\left(Z_{j}\right)\right)\right)
\end{array}
$$

with $\tilde{K}$ as given by (4.5). Combining (A.25), (A.26), (A.18) and (A.19) we see that (A.15) and Corollary A.1 are proved.

\section{REFERENCES}

Albers, W. (1974). Asymptotic Expansions and the Deficiency Concept in Statistics. Mathematical Centre Tracts 58, Amsterdam.

Albers, W., Bickel, P. J. and van Zwet, W. R. (1976). Asymptotic expansions for the power of distribution free tests in the one-sample problem. Ann. Statist. 4 108-156.

BiCKel, P. J. (1974). Edgeworth expansions in nonparametric statistics. Ann. Statist. 2 1-20.

EFron, B. (1975). Defining the curvature of a statistical problem (with applications to second order efficiency). Ann. Statist. 3 1189-1217.

EsSEen, C. F. (1945). Fourier analysis of distribution functions. A mathematical study of the Laplace-Gaussian law. Acta Math. 77 1-125.

HÁJEK, J. (1964). Asymptotic theory of rejective sampling with varying probabilities from finite populations. Ann. Math. Statist. 35 1491-1523.

Hodges, J. L. and Lehmann, E. L. (1963). Estimates of location based on rank tests. Ann. Math. Statist. 34 598-611.

Hodges, J. L. and Lehmann, E. L. (1970). Deficiency. Ann. Math. Statist. 41 783-801.

Окамото, M. (1958). Some inequalities relating to the partial sum of binomial probabilities. Ann. Inst. Statist. Math. 10 29-35.

Pfanzagl, J. (1977). First order efficiency implies second order efficiency. To appear in J. Hájek Memorial Volume, Academia, Prague; North-Holland, Amsterdam.

RÉnyI, A. (1970). Probability Theory. North-Holland, Amsterdam.

Robinson, J. (1978). An asymptotic expansion for samples from a finite population. Ann. Statist. 6 1005-1011.

Rogers, W. F. (1971). Exact null distributions and asymptotic expansions for rank test statistics. Technical Report 145, Departments of O.R. and Statistics, Stanford Univ.

RyshiK, I. M. and Gradstein, I. S. (1957). Tables of series, products and integrals. V.E.B. Deutscher Verlag der Wiss., Berlin.

Department of Statistics

UNIVERSITY OF CALIFORNIA

Berkeley, California 94720
UNIVERSITY OF LEIDEN

Department of Mathematics

WASSENAARSEWEG 80

P.O. BOX 9512

2300 Ra LEIDEN

The Netherlands 